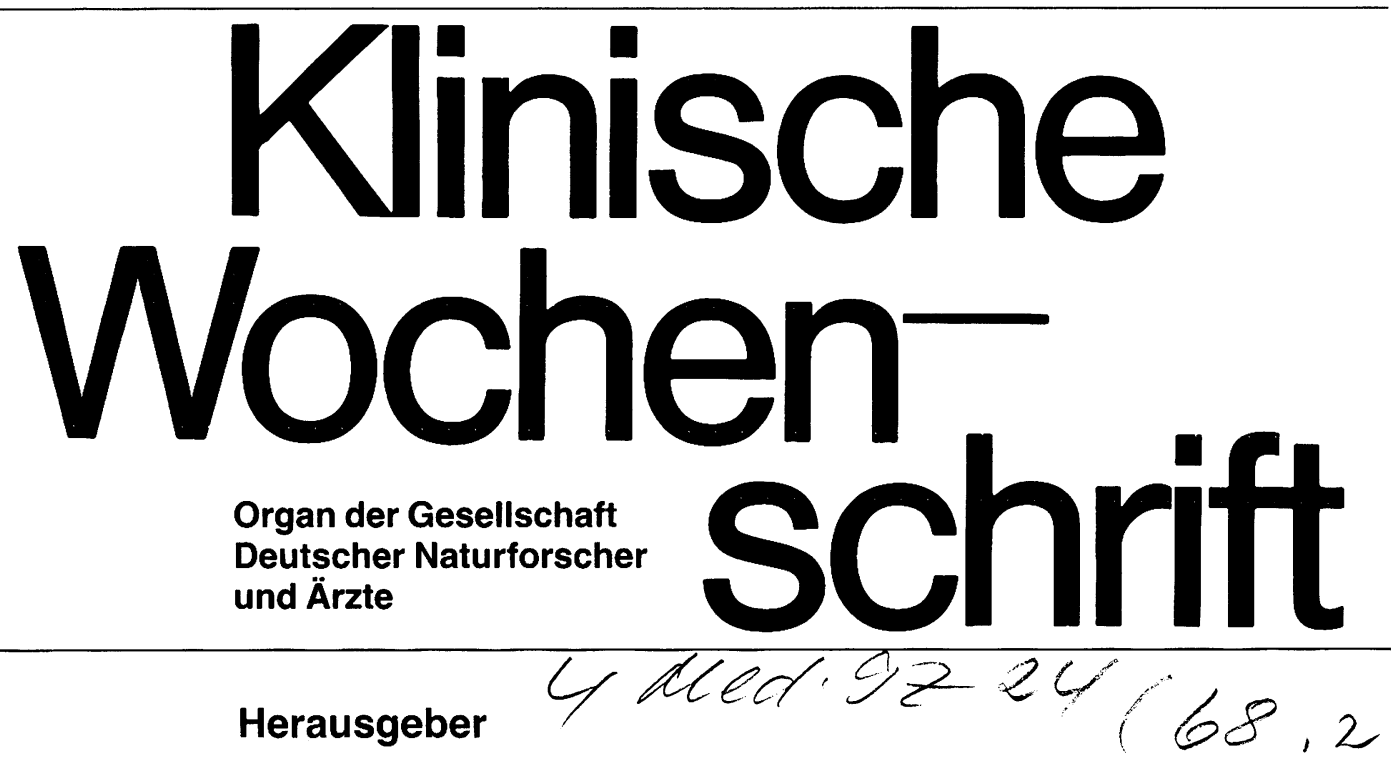

Schriftleitung

N. Zöilner
E. Buchborn (München) · H.J. Dengler (Bonn) • V. Diehl (Köln) W. Gerok (Freiburg) · H. Goebell (Essen) · R. Gross (Köln) $H$. Jahrmärker (München) $\cdot H$. Kaess (München) · F. Krück (Bonn) W. Kübler (Heidelberg) · H.-G. Lasch (Gießen) · F. Loogen (Düsseldorf) · W. Lorenz (Marburg) · P.A. Miescher (Genf) G. Paumgartner (München) · W. Reutter (Berlin) · G. Riecker (München) · G. Schettler (Heidelberg) · P. Schölmerich (Mainz) · P.C. Scriba (München) · D. Seidel (München) · G. Seifert (Hamburg) · G.H. Thoenes (München) · P. von Wichert (Marburg) N. Zöllner (München)

\section{Beirat}

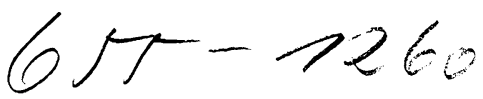

R. Arnold (Marburg) · A. Distler (Berlin) · R. Ferlinz (Mainz)

O. Götze (Göttingen) · F.A. Gries (Düsseldorf) · H.-L. Hahn (Würzburg) · W.H. Hitzig (Zürich) · J. Köbberling (Wuppertal)

E. Köttgen (Berlin) · H.J. Kramer (Bonn) · H. Löffler (Kiel)

B. Lüderitz (Bonn) · R. Lüthy (Zürich) · M. Marshall (Tegernsee)

K.-H. Meyer zum Büschenfelde (Mainz) · M.J. Mihatsch (Basel)

H. Mörl (Mannheim) $\cdot$ Ch. Mueller-Eckhardt (Gießen)

B.M. Peskar (Bochum) · E.O. Riecken (Berlin) · R. Ritz (Basel)

M. Schattenkirchner (München) · W. Schneider (Düsseldorf) H.-P. Schuster (Hildesheim) · W. Seeger (Gießen) · H.-G. Sieberth (Aachen) - W. Stille (Frankfurt) - K.O. Stumpe (Bonn)

K.-H. Usadel (Mannheim) · G. Utermann (Innsbruck) · C. Wagener $($ Hamburg) $\cdot$ I. Walter-Sack (Heidelberg) · E. Weber (Heidelberg)

K. von Werder (Berlin) · G. Wolfram (München)

\section{Redaktion Pharmakologika}

E. Weber, I. Walter-Sack (Heidelberg) 


\section{Sachregister}

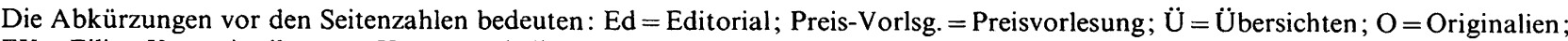
$\mathrm{EK}=$ Eilige Kurzmitteilungen; $\mathrm{Ka}=$ Kasuistik; Pharma = Pharmakologika $; \mathrm{Bespr}=$ Buchbesprechungen; Brfe $=\mathrm{Briefe}$ an die Herausgeber; Kon $=$ Kongresse $;$ Suppl $=$ Supplementum .

Abamectin Bespr 926

AB-Amyloid Kon 1150

Abbott TDx fluorescence polarisation immunoassay Ka 325

Abdomen, komplexe bildgebende Diagnostik Bespr 1075

Abdominal pain Ü 247

Abgeschlagenheit 089

Abgespanntheit O 969

Abstoßungskrisen O 408, O 409

Abstoßungsreaktion $\mathrm{O} 410$

ACE inhibition $\mathrm{O} 750$

Acetatehemodialysis $\mathrm{O} 806$

Acetyl-choline O 166, O 172

Acetylcholinesterase $\mathrm{Ka} 615$

Acetylcholininduzierte Desensibilisierung Ka 100

Acetylsalicylic acid EK 294, O 1059

Acid base regulation Kon 1096

Acid-base homeostasis O 175, Kon 1096

Acid-base status O 175

Acid-clearance of the tubular esophagus $\mathrm{O} 602$

Acidosis O 180

Acid phosphatase Ka 95

Acquired immunodeficiency syndrome $\mathrm{O} 43$

Acromegaly O 583

ACTH-Test Ka 329

Activated partial thromboplastin time Ka 1071

Acute Cyclosporine A tocicity O 835

Acute Guillain-Barré syndrome O 1183

Acute leukemias $\mathrm{Ka} 874$

Acute liver disease $\mathrm{O} 178$

Acute myocardial infarction O 774, O 1174

Acute occlusive deep venous thrombosis $\mathrm{O} 1208$

Acute pancreatitis $\mathrm{O} 565$

Acute renal failure 0758

Acute uric acid nephropathy Ka 874

Acyclovir Ka 286, Ka 1190, Ka 1193

Adeninnukleotid O 1179

Adenoma $\mathrm{Ka} \mathrm{342,} \mathrm{Kon} 1102$

Adenomas of the anterior pituitary 0669

Adenome, tubulovillöse O 87

Adenom-Karzinom, Abgrenzung Kon 11107

Adenosindiphosphat $\mathrm{O} 460$

Adenosine-triphosphate $\mathrm{O} 223$

Adenosinmonophosphat, cyclisches Ka 927

Adenylatcyclaseregulation Ü 890

Adenylate cyclase Ü 54, Ü 887, Ka 927

Adenylzyklase O 681

ADH secretion Ka 191

Adhesion molecules Bespr 1177

Adrenal adenoma Ka 981

Adrenal androgens $\mathrm{O} 597$

Adrenal glands $\mathrm{Ka} 1027$

Adrenal hyperplasia, clinical symptomes Ka 328

-, laboratory findings $\mathrm{Ka} 328$

Adrenal hypofunction $\mathrm{Ka} 1076$

Adrenal insufficiency Ka 927, Ka 1076

Adrenal neoplasm Ka 1076

Adrenal tumor Cushing's syndrome O 608

Adrenalektomie Ka 328, Ka 1077
Adrenalin-Sekretion Ka 99

Adrenals Ü 699, Ü 700

$\beta$-adrenergic blockade EK 441

Adrenergic receptors O 557

$\beta$-adrenic influence on the heart 026

$\beta$-adrenoceptor blockade $\mathrm{O} 476$

$\beta$-adrenoceptors $\ddot{U} 887$

Adrenocorticotropes Hormon Ka 329, Ka 927

Adrenocorticotropic hormone O 951

Adrenocorticotropin O 608

Adrenogenitales Syndrom Ka 328

$\beta$-Adrenozeptor-Agonisten Ü 893

$\beta$-Adrenozeptor-cAMP-Adenylatcyclasesystem Ü 893

$\beta$-Adrenozeptoren Ü 890

$\beta_{1}$-Adrenozeptoren Ü 893

$\beta_{2}$-Adrenozeptoren Ü 893

Adriamycin Ü 443

Adult respiratory distress syndrome $\mathrm{O} 1183$

Aerodermatitis $\mathrm{Ka} 431$

Aerosols O 397

Afferent inhibition EK 125

Aflatoxin B1 Ü 443

Africa 0277

Age 0655

Aggregationsverhalten der Thrombozyten $\mathrm{O} 462$

AIDS O 43, O 89, Ü 299, O 393, O 415, O 853, O 864, O 906, O 1003, Ka 1034, O 1050, O 1203, EK 1229

-, bildgebende Diagnostik Bespr 475

AIDS-related complex O 89, O 393, O 1050

Air embolism O 1187

Akromegalie, IGF-I Spiegel Ü 993

Albright's hereditary osteodystrophy Ka 930

Albumin O 1184

Albumine 0678

Alcohol O 655

Alcohol consumption O 655

Alcoholism Ka 191, O 678

Aldactone Ü 347

Aldosterone O 71, O 74, O 313, O 476, O 583, Ka 981, Kon 1087, Kon 1127

Alkalosis O 180

Alkoholkonsum O 655

Alkoholreduktion $\mathrm{O} 662$

Alkylphosphat-Intoxikationen Ka 100

Allograft rejection O 869

Alopecia totalis $\mathrm{Ka} 237$

Alpha-adrenoceptor antagonist Pharma 624

Alpha-1-acid glycoprotein Pharma 624

Aluminum $\mathrm{O} 41$

Alveolar ventilation $\mathrm{O} 806$

Alveolitis O 512

Amaurose Ka 333

Amblyopia Ka 332

Amblyopie $\mathrm{Ka} 333$

Ambulatory blood pressure monitoring O 723, Kon 1119

Amino acid metabolism O 380

Amino acids O 750, Ü 1157

Aminoglutethimid $\ddot{U} 354$ 
Aminoglycoside antibiotics Pharma 624

Aminoglycosides O 869

Aminosäurestoffwechsel O 380

Aminotransferase $\mathrm{O} 184$

Amiodarone $\mathrm{O} 673$

Ammonia Kon 1096

Ammonium O 175

Amoxicilline Ka 1032

AMP Kon 1138

Amphotericine O 869

Amputation O 380

Amylase O 565

Amyloid angiopathy $\mathrm{O} 1173$

Anal erosion Ka 523

Anal intercourse O 906, O 1205

Analgesia EK 129

Analgesic abuse O 38

Analgesic nephropathy O 38, O 1042

Analgesic syndrome O 38

Analgesic-associated nephropathy $\mathrm{O} 40$

Analgesics O 38, EK 121

Analgetika, nichtsteroidale EK 121

Analgetika-Mißbrauch O 1044

Analgetikanephropathie O 1042

Analysis of intracellular parameters Ü 199

Anämie $\mathrm{O} 89$

-, korpuskuläre hämolytische Ü 248

Anandron Ü 347

Androcur Ü 347

Androgene Ü 250

Androgenic stimuli Ü 347

Androgen-Synergismus, Störung Ü 355

Androgen-Synthese, Hemmung Ü 354

Androgen-(Testosteron-)Entzug Ü 351

Androgen-Wirkung auf die Target-Zelle, Hemmung Ü 354

Androstendion Ka 329

Anemia O 24, O 41, O 403, O 405, O 1050

Anesthesia O 1172

-, general O 1168

ANF O 313, O 823

Angina pectoris O 1185

Angiodysplasia Ka 290

Angioedema $\mathrm{Ka} 51$

Angiogram Ka 619

Angiography Ka 1195, Ka 1197

-, intraarterial Ka 1196

Angiological examination O 589

Angiopathy EK 49

Angiotensin converting enzyme Ka 335, O 750

Angiotensin I-Converting-Enzyme Ka 340

Angiotensin II O 758, Kon 1127, Kon 1138

Angiotensine O 583

Angstanfälle Bespr 1202

ANP O 476, Ü 699, Kon 1112

ANP levels O 476

ANP mRNA Ü 699

Anterior uveitis Ka 971

Antiandrogens Ü 347

Antiarrhythmic drugs Bespr 1026

Antiarrhythmika O 1181

Antibiotic therapy O $372, \mathrm{Ka} 427, \mathrm{Ka} 431$

Antibiotika-Therapie Bespr 522

Antibodies O 685

Antibodies against cycloplasmic components O 685

Antibodies against mistletoe extract O 896

Antibody activities O 685

Antibody levels O 512
Antibody-HIV-1 immune complexes Ü 301

Anticardiolipin antibodies O 594

Anticholinerger Effekt O 1181

Anticholinergics Ü 935

Anti-CMV-IgG Ka 237

Anti-CMV-IGM Ka 237

Antidiuretic hormone O 313

Antigen-antibody reaction $\mathrm{O} 35$

Antigenic stimulation Ka 95

Anti-HIV-1 seronegatives $\mathrm{O} 43$

Anti-HIV-1 seropositives O 43

Antihypertensive drugs $\mathrm{O} 768$

Antihypertensive Therapie Kon 1124

Antihypertensive therapy O 320,0718

Antihypertensives, long acting O 320

-, rapidly acting $\mathrm{O} 320$

Anti-inflammatory analgesics EK 121

Antikoagulation O 1213

Anti-lectin-antibodies O 896

Antilymphozytenglobulin Ka 1189

Antimosquito measures O 277

Antineoplastic agents, intrathecal application Ka 1076

Antineopolastic therapy $\ddot{U} 8$

Antinociception EK 129

Antiphospholipid antibodies O 594

Anti-p24-antibodies 0864

Antirejection therapy O 408

Antirheumatika, nichtsteroidale EK 121

Antistreptolysin-0-titer Ka 1198

Antithrombin III O 559

Antithymocyte globuline $\mathrm{O} 408$

Antithyroid drugs O 1037

$\alpha_{1}$-antitrypsin deficiency EK 346

$\alpha_{1}$-antitrypsin O 857

Antitumor drugs $\ddot{U} 443$

Anxiety 0942

Aorta O 570

Aortic valve disease $\mathrm{O} 921$

apo E2 O 67

Apoenzymes O 183

Apolipoprotein E O 65, Ka 526

Apoplex O 521

Arachidonic acid Ü 56

ARC O 1203

Arcuate arteries O 166, O 170

ARGUS-100 O 33

Arrhythmias O 1171

Arrhythmiebehandlung Bespr 517, Bespr 1216

Arterial hypertension $\mathrm{O} 41$

Arterial occlusive disease O 207

Arterial pressure $\mathrm{O} 718$

Arterial smooth muscle cells Ü 53

Arterielle Verschlußkrankheit O 380

Arterieller Hochdruck O 1047

Arteriosclerosis O 518

Arteriosklerose O 518

-, Frühdiagnostik $\mathrm{O} 521$

Arterio-venöse Fistel O 1208

Arteriovenous shunting O 210

Artery-to-artery embolism O 1176

Arthralgia $\mathrm{Ka} 739$

Arthritis $\mathrm{Ka} \mathrm{431,} \mathrm{Ka} 881$

-, rheumatoid Ü 56, O 460

Arthropathy Kon 1150

Artificial endocrine pancreas O 951

Artificial pancreas Ü 306

Arylesterases 060 
Arzneimittelprüfung, Psychiatrie Pharma 1085

Ascites O 545

Aspartate O 223

Asphyxien O 1179, O 1181

Assoziation zwischen Hautfibromen und Darmpolypen O 86 Asthma Suppl XIX

Asystole O 1187

$\alpha 1$ AT deficiency alleles Pi QO, Pi Z O 857

Atemwegserkrankungen, obstruktive Suppl XIX

Atherogenesis Ü 53, 55

-, rheumatic disease Ü 56

Atherogenic serum cholesterol fraction O 269

Atherosclerosis Suppl XX, O 38, O 41, O 65, O 570

Atherosclerotic affected tissues EK 49

Atherosclerotic lesions O 68, O 570

Atherosclerotic vascular disease $\mathrm{O} 664$

Atherothrombotic infarction O 1176

ATP detection O 33

ATP-dependent efflux pump Ü 444

Atrial fibrillation O 1173, O 1174, O 1186

Atrial natriuretic factor O 313, O 709, O 823

Atrial natriuretic peptide EK 441, Ü 699, O 758, Kon 1112, Kon 1127

Atrial natriuretic peptide in peripheral organs $\ddot{U} 699$

Atrialer natriuretischer Faktor $\mathrm{O} 823$

Atriales natriuretisches Peptid O 476

Atrioventricular block $\mathrm{Ka} 431$

Atropine $\mathrm{Ka} 615$

Auricular trabeculae 0797

Autoantibodies O 1066

Autocoids Kon 1138

Autoimmune diseases Suppl XXI, Bespr 402, Ü 489

Autoimmune etiology O 1183

Autoimmune hemolytic anemia Ka 94

Autoimmune hyperlipidemia Ka 526

Autoimmune hyperthyroidism O 942

Autonomic cardiovascular control O 30

Autonomic dysfunction O 1171, O 1172

Autonomic neuropathy O 30

Autopsy O 1173

av-Fistel O 1213

Azathioprin Ka 1189, Ka 1190

Azathioprine Ka 50, Ka 1071

B Vitamin EK 142

Baclofen Ü 938

Bacterial infections O 14, O 218

Bacterial infections of the CNS O 12

BAL O 853

Balloon angioplasty $\mathrm{O} 570$

Bam H I EK 1084

Bannwarth syndrome $\mathrm{Ka} 431$

Barbiturates O 1172

BARD (In 37) Normotherm System Ka 620

Baroreceptor response Pharma 625

Basement membrane Kon 1145

Baxter-Stratus fluorescence enhanced enzyme partition immunoassay $\mathrm{Ka} 325$

Bayes method O 469

B-cell lymphoma Ka 1027

B-cell neoplasms O 16

B,E (LDL)-receptors O 68

Befeuchterlunge O 512

Befeuchterwasser, mikrobiologische Untersuchung O 515

Behandlung mit B-Vitaminen EK 146

Behçet's syndrome Ü 493, O 685

Beinvenenthrombose, tiefe O 1208
Belastung, körperliche $\mathrm{O} 827$

Benign monoclonal gammopathy Ka 95

Benzbromarone $\mathrm{Ka} 878$

Benzbromarone elimination EK 101

Benzodiazepines Pharma 624

Bestrahlung, lokale Ka 1189

Beta-adrenoceptor antagonists Pharma 624

Beta-blockers Pharma 624, O 951

Betaine Kon 1091

Betaxolol O 476

Beta-2-microglobulin O 864

Bezafibrate O 65

Bezafibrate therapy O 65

Bicarbonate Kon 1096

Bicarbonate elimination Kon 1096

Bicarbonate homeostasis O 175

Bicarbonatehemodialysis O 806

Bile analysis O 497

Bile canalicular membranes Ü 444

Bile duct lithiasis O 496

Bile infection $\mathrm{O} 500$

Bile salts O 60, O 63, Ü 443

Biliary excretion O 692

Bilirubin plasma concentration O 692

Biological membranes Preis-Vorlsg. 149

Bioluminescence O 33

Blast crisis O 23

Blastocyst cells Ü 53

Bleeding tendency Ka 1071

Bleomycin Ü 539

Blepharospasm Ü 939

$\beta$-blockers Ü 887

Blood O 507

Blood cells Bespr 396

Blood donors O 1222

Blood Epo level O 405

Blood flow of reactive hyperemia O 590

Blood glucose Ü 306, O 447

Blood glucose monitoring Ü 306

Blood hemoglobin concentration $\mathrm{O} 406$

Blood platelets $\mathrm{O} 460$

Blood pressure O 173, O 320, O 718, O 768, O 1168, O 1171, O 1172, Ka 1198

-, instability O 1168

- , lability O 1171

Blood pressure monitoring O 320, O 723

$24 \mathrm{~h}$ Blood pressure profiles $\mathrm{O} 320$

Blood Pressure Regulation Kon 1112

$24 \mathrm{~h}$ blood pressure $\mathrm{O} 723$

Blood sugar levels O 951

Blood urea nitrogen $\mathrm{O} 65$

Blood velocity O 173

Blood viscosity O 23

Blood-brain barrier Pharma 624

Blood/membrane interactions O 901

Blutdruck O 655

Blutdruckabfall, nächtlicher Kon 1121

Blutdruckanstieg, morgendlicher O 320

Blutdruckregulation Kon 1112

Blutdruckrhythmik Kon 1121

Blutdruckselbstmessung Kon 1123

Blutdrucksenkung O 662

BMI O 655

Body fat Pharma 623

Body fluid volume Kon 1112

Body mass Pharma 623

Body mass index $\mathrm{O} 655$ 
Body water Pharma 623

Body weight Ka 1198

Boeck-Besnier-Schaumann-Erkrankung Ka 340

Boeck's disease Ka 344

Bolus O 709

Bone marrow O 901

Bone marrow depression O 1040

Bone marrow failure $\mathrm{O} 403$

Bone marrow transplantation O 406, Ü 539

Bone metastases $\mathrm{Ka} 388$

Bone mineral content Ü 359

Bone-marrow disease Ü 247

Borborygmi O 606

Borrelia burgdorferi spirochete Ka 431

Botulinum toxin Ü 935, Ü 938

Bradycardia O 1186

Bradycardia syndrome O 313

Brain edema Ka 286

Brain metastases O 576

-, surgical treatment O 576

Breast feedings Ü 1157

Bromocriptin Ü 349, Ka 384

-, Nebenwirkungen Ka 386

Bronchialkarzinom Suppl XIX, O 579

-, kleinzelliges Ka 244

Bronchitis Suppl XIX

Bronchoalveolar lavage O 372, O 853

Bronchoalveolar lavage fluid $\mathrm{O} 728$

Bronchoscopic methods, nonbioptic O 372

Bronchoscopy O 372

Bronchoskopie, flexible O 372

Bronchoskopisch nachgewiesene Erreger O 374

Bronchoskopische Untersuchungen, Komplikationsrate O 376

Bronchoskopische Untersuchungsverfahren, nicht bioptische O 372

Bronchospasm O 1185

Brown pigment stones $\mathrm{O} 496$

Bundle branch block Ü 744

Burkitt-Lymphom Ka 1080

Butyl nitrite O 906

B-vitamin EK 125

B-Zell-Hyperplasien Ka 1189

B-Zell-Lymphome Ka 1076, Ka 1189

Calcitonin Ü 359

-, exogenes Ü 362

Calcitonin analgesia Ü 359

Calcitonin receptors in the brain $\ddot{U} 359$

Calcitonin secretion Ü 359

Calcitoninmangel Ü 361

Calcitoninreserve Ü 361

Calcitoninstimulierbarkeit Ü 361

Calcitoninwirkungen, unerwünschte Ü 365

Calcium Ü 359, O 472, O 718, Ü 893

Calcium metabolism $\mathrm{O} 421$

Calcium-Antagonist Bespr 580, Bespr 984

Calcium-channel blocker O 166

Calcium-Hormone, endogene Ü 363

Calcium-/Phosphat-Homöostase Ü 363

Calcium-Rückresorption O 682

cAMP Kon 1138

cAMP-Adenylatcyclasesystem Ü 887

cAMP-phosphodiesterase inhibitor Ü 887

Cancer Ü 1 , Ü 53, Ü 56

Cancer Research Bespr 1194

Cancer treatment Ü 8, Bespr 536

-, adjuvant therapy O 896
Candida esophagitis Ka 187

Captopril O 750

Carbamazepin Ka 1193

Carcinoid, metastasiertes Ka 241

Carcinoid-Syndrom Ka 244

Carcinomas Kon 1102

Cardiac arrest O 1185

Cardiac arrhythmia O 1185, O 1186, O 1187

Cardiac failure $\mathrm{Ka} 880$

Cardiac function, autonomic nervous control of O 26

Cardiac glycosides Ü 887

Cardiac output O 263

Cardiac rhythm disorders O 1168

Cardiac surgery, perioperative mortality O 1059

Cardiogenic shock O 313

Cardiomyopathy O 914, O 1174

Cardiopulmonary instability O 806

Cardiosurgical interventions O 921

Cardiovascular complications O 1186

Cardiovascular disease O $38, \mathrm{O} 65, \mathrm{O} 458$

-, risk factors $\mathrm{O} 458$

Cardiovascular instability O 1172

Cardiovascular morbidity $\mathrm{O} 38, \mathrm{O} 41$

Cardiovascular reactions, perioperative $\mathrm{O} 1170$

Cardiovascular reflectory reactions O 1168

Cardiovascular reflectory status O 1168

Cardiovascular system O 1168

Carmustine Ü 539, Ü 542

Casodex Ü 347

Catecholamines Bespr 182, O 271, O 951, Kon 1127

$\mathrm{Ca}^{2+}$-antagonists $\mathrm{O} 797$

CDC-classification 089

cDNA O 914

$\mathrm{CD}^{+}$cells $\mathrm{O} 728$

CD3 + HLA/DR + cells O 395

CD4 cells O 393, O 728, O 1203

CD4/CD8-Lymphozyten-Quotient O 855

CD4/CD8-ratio O 408

$\mathrm{CD} 4+/ \mathrm{CD} 8+\mathrm{T}$-cell ratio $\mathrm{O} 44$

CD4-positive T-Helfer-Lymphozyten O 855

CD4 + T-cells O 44

$\mathrm{CD}^{+}$cells O 1203, O 1206

CD8 lymphocytes O 393

CD8 + Leu 7 + cells O 395

CD8-positive zytotoxische T-Zellen O 855

CD8 + T-cells O 44

$\mathrm{CD}_{16}{ }^{+}$cells $\mathrm{O} 728$

Ceftriaxone Ka 431

Cefuroxim Ka 1032

Celiac disease Ü 493

Cell growth Kon 1087

Cell lines Ü 53

Cell measurement O 394

Cell preparations O 394

Cell surface antigens $\mathrm{O} 408$

Cell turnover O 518

Cell-surface ruffling Ü 54

Cellular and antigen-antibody reactions $\mathrm{O} 33$

Cellular immunity $\mathrm{O} 728$

Cellulose-based membranes O 901

Central nervous system O 12, Ü 53

Central venous pressure $\mathrm{O} 313$

Cerebellar hemorrhage $\mathrm{O} 1174$

Cerebral embolism O 921

Cerebral hemorrhage O 1173, O 1174, O 1175

Cerebral infarction O 1173, O 1174, O 1176

Cerebral ischemia O 1187 
Cerebral lymphoma Ka 1189

Cerebral white matter O 1176

Cerebrospinal fluid O 12, Ka 485, O 1054

Cerebrovascular disease O 1173

Chemiluminescence $\mathrm{O} 33$

Chemotaxis Ü 54

Chemotherapie Ka 332, Ka 1077

Chemotherapy Ü 443, Bespr 536, Ü 539, Ka 874, Ka 880

Chief cell adenoma O 472

Chlamydia infection $\mathrm{Ka} 971$

Chlorid O 661

Chloride Ü 199

Chloroquine resistance O 277

Chlorpromazine Pharma 624

Cholecystectomy O 496

Cholelithiasis O 496

Cholesterinkonzentration O 85, O 86

Cholesterol O 65, O 269, O 454, O 664, O 1183

Cholesterol education program $\mathrm{O} 454$

Cholesterol ester hydrolase O 60, O 63

Cholesterol esterase O 60

Cholesterol levels O 455

Cholesterol stones O 496

Cholesterol-esterase-dependent oral test O 60

Cholestyramine O 816

Choline Kon 1091

Cholinerge Rezeptoren Ka 99

Cholinesterase inhibitors Ka 615

Cholinesterase-inhibitor poisoning Ka 615

Choriongonadotropin Ka 244

Chromaffine Zellen $\mathrm{Ka} 99$

Chromogranin A Ka 244

Chromophobe cell carcinoma Kon 1102

Chromosome 6 Ü 1157

Chronic alcoholism Ka 187

Chronic fatigue syndrome Ü 789

Chronic hemodialysis O 38,065

Chronic hypertension O 559

Chronic inflammatory diseases Ü 56

Chronic liver disease O 175, O 180

Chronic lymphocytic leukemia Ka 95

Chronic mononucleosis Ü 789

Chronic obstructive lung disease 0857

Chronic renal failure O 65, O 183, O 405, O 692

Chylomicrons O 67

Ciclosporin, hepatic metabolism EK 872

Ciclosporin-Propafenone interaction EK 872

Cimetidin Ü 354

Cimetidine Pharma 624

Circadian rhythm of blood pressure Kon 1119

Circadian rhythm of cortisol levels O 1217

Circulating immune complexes Ü 299

Circulatory system O 1168

Cirrhosis O 959, $\mathrm{Ka} 1032$

Cirrhotic ascites O 545

Cisapride O 602

Class-I antiarrhythmic drugs O 1178

Clusters of Differentiation Bespr 217

CMV Ka 237

CNS EK 136, EK 142

Coagulation Ka 281

Coagulation disorder O 1185

Coagulation factor IX Ka 281

Colipase O 60, O 63

Collagen O 460

Collecting duct Ü 199

Colloid osmotic pressure O 559
Colon carcinoma $\mathrm{O} 83$

Colonic polyps $\mathrm{O} 83$

Colony stimulating factors Kon 1138

Color blood flow imaging Bespr 536

2-color flow cytometry O 408

Colorectal carcinomas Ü 443

Coloscopy $\mathrm{O} 83$

Coma Ka 615, O 1037

Common bile duct calculi O 496

Complement O 1066

Complement factor $3 \mathrm{Ka} 1198$

Complement factor $4 \mathrm{Ka} 1198$

Complement regulation $\ddot{U} 247$

Complementary desoxyribonucleic acid O 914

Computed tomography Ka 328 , Ka 485

Computer assisted diagnosis $\mathrm{O} 466$

Computer reasoning O 466

Computer tomography Ka 191

Computertomographie Bespr 435, Ka 1192

Computertomographie des Abdomens Bespr 773

Congenital adrenal hyperplasia Ka 328

Congenital heart disease O 1174

Congophilic deposits O 1174

Conjunctivities Ka 881

Contraception, barrier O 1205

Contractile reserve of the failing myocardium Ü 887

Coombs' test O 1050

Coombs-positive warm-type autoimmune hemolytic anemia $\mathrm{Ka} 94$

Coombs-Test Ü 250

Cooperative behavior O 397

Copper O 507

Corneal graft rejection $\mathrm{O} 869$

Corneal transplantation O 869

Coronary angioplasty EK 294, O 823

Coronary artery disease EK 294, O 594, O 774, O 801

Coronary bypass surgery $\mathrm{O} 774$

Coronary heart disease O 41, O 664, O 1059

Coronary risk factors O 594

Coronary stenoses O 594

Coronary vascular disease $\mathrm{O} 68$

Cortical infarction O 1174

Corticosteroid treatment Ka 1027

Corticosterone Ka 981

Corticotropin releasing hormone O 608

Corticotropin-Releasing Hormon Ka 927

Cortisol O 951, Ka 981

Cortisol levels O 1217

Cortisoldefizit Ka 330

Cortisoltagesprofil Ka 328

Cortison Ka 1190

Cortissol response O 608

Cox proportional hazards regression model 0869

Cox regression O 397

Coxsackie-B virus Ü 789

Coxsackievirus B3 O 914

Cramps/paraesthesias O 1185

Crash fractures of vertebras $\ddot{U} 359$

C-reactive protein Ka 976, EK 1083

Creatinine O 65, O 503

Creatinine clearance O 256, O 750

Creatinine kinase O 65

Cremophore O 173

Crohn's disease O 685

Cross resistance Ü 443

Cryopallidotomy Ü 939

Cryothalamotomy Ü 939 
Cs-A-Serumspiegel Ka 1191, Ka 1193

CSF-1 Kon 1138

${ }^{13} \mathrm{C}$-Spektroskopie Ü 200

CT Bespr 435

CT-scan Ka 1196

Cuprophane O 901

Cushing's syndrome O 608, Ka 981

Cyclic adenosine monophosphate $\mathrm{O} 678$

Cyclic GMP O 213, O 709

Cyclic guanosine monophosphate O 476

Cyclisches Adenosin-3'5'-monophosphat Ü 887

Cycloheximide Ü 443

Cyclophosphamid Ka 1077

Cyclophosphamide Ü 539, Ka 976

Cyclosporin O 869

Cyclosporin A O 166, Ka 1193

Cyclosporin A-Therapie O 835

Cyclosporin nephrotoxicity O 869

Cyclosporin-A Ka 1190

Cyclosporin-A-Reduktion Ka 1189

Cyclosporine A O 408, Ka 1189

Cyclosporin-Therapie Ka 1192

Cyproteronazetat Ü 347, O 597

Cyproterone acetate O 597

Cystic neoplasm Ka 780

Cytapheresis O 18

Cytarabine Ü 542

Cytochrome P-450 EK 872

Cytokines Ư 1, Ü 7, Ü 8, Bespr 536, Ü 841, Bespr 1065, EK 1083, Kon 1138

-, recombinant human $\ddot{U} 8$

Cytology in Transplantation Bespr 430

Cytomegalovirus Ka 237, O 906, O 1003, O 1050

Cytomegalovirus infection diagnosis $\mathrm{O} 1003$

Cytomegalovirus-specific recombinant polypeptide O 1003

Cytoplasmic pH Kon 1087

Cytosin-Arabinosid Ka 333

Cytoskeleton O 685

Cytosolic calcium $\mathrm{O} 718$

Cytostatic drug O 24

Cytostatic drugs Ü 8

Cytostatic treatment O 406

Cytotoxic cells Ü 1, Ü 7

Cytotoxic effect of drugs $\ddot{U} 443$

Cytotoxic T lymphocytes Ü $1, \ddot{U} 3$

Cytotoxic tumor infiltrating lymphocytes Ü 3

Cytotrophoblast cells of placenta Ü 53

C1q O 1066

C1q-binding capacity Ka 1198

C1q-binding immune complexes 0864

C1q-Solid-Phase-Bindingassay O 1066

Dacarbazine Ü 539

Darmpolypen O 83

Debrisoquine O 269

Decay accelerating factor Ü 247

Decision analysis Ed 297

Decrease of arterial $\mathrm{pO}_{2} \mathrm{O} 806$

Decreased ability to concentrate 0947

Deep vein thrombosis O 1020

-, fibrinmonomer test O 1020

Deep venous thrombosis O 1208

Degegenerative und regenerative Vorgänge an peripheren Nerven EK 146

Dehydroepiandrosteron-Sulfat Ka 329

Delayed gastric emptying O 961

Demyelinisation Ka 191
Denervation supersensitivity O 1171

Dental surgery, bacteremia O 924

Depression O 942

Depressivität O 969

Dermatological diseases O 1183

Desoxyribonucleic acid Ü 1157

Deutsche Gesellschaft für Innere Medizin, 96. Tagung Suppl XIX

Dexamethason O 597

Dexamethasone Ü 542, O 597

Dexamethasone suppression test 0608

Diabetes insipidus Kon 1091

Diabetes mellitus O 26, O 77, Bespr 102, Ka 187, Ü 199, O 591, O 923, Ü 985, Kon 1091

-, IGF-Spiegel Ü 996

-, vascular complications Bespr 537

Diabetes mellitus type I O 951

Diabetesbehandlung Ü 306

Diabetic autonomic neuropathy O 1168 , O 1171

Diabetic foot O 588

Diabetic microangiopathy O 207

Diabetic neuropathy 026

Diabetics O 26, EK 49, O 588, O 1168, O 1171

Diabetologic knowledge O 77

Diagnosis of opportinistic pulmonary infections O 372

Diagnostik, medizinische Ed 297

-, medizinisch-klinische Bespr 453

Diagnostik und Therapie, Obsoletes Suppl XIX

Dialysepatienten Kon 1150

Dialysis Ka 324, Kon 1150

Dialysis treatment $\mathrm{O} 38, \mathrm{O} 40$

Diameters O 170

Diarrhea Ka 50 , Ka 525

Diarrhoe O 89

Diastolic blood pressure 0709

Diätetik Bespr 525

Diätik Bespr 392

Diazepam Pharma 624

Diclofenac EK 107, EK 116, EK 121

Dietary fat Suppl XX

Dietary salt restriction O 664

Differential diagnosis, multiple endocrine neoplasia syndromes O 669

Differentialdiagnose Bespr 296

Differentialdiagnostik bei hyperthyreoter Stoffwechsellage Pharma 635

Digital substraction angiography $\mathrm{O} 830$

Digitalis antibody Fab-fragment therapy Ka 324

Digitalis antibody fragments Ka 324

Digitoxin poisoning $\mathrm{Ka} 324$

Digoxin Pharma 624

1,4-dihydropyridine $\mathrm{O} 797$

Dihydrotachysterol O 681

1,25-Dihydroxyvitamin D O 421

Dilated cardiomyopathy O 914

Diltiazem O 797

Direct antiglobulin test O 1050

Direct DNA sequencing O 857

Disgerminomas O 16

Disopyramid O 1180

Disopyramide Pharma 624, O 1178

Dispnea O 1185

Disseminated carcinoma Ka 874

Disseminated intravascular coagulation O 1020

Disseminated lupus erythematosus Ka 976

Dissolution of CyA O 173

Diuresis Kon 1150 
Diuretic treatment O 178

Diuretic-induced lipid disturbances 0664

Diuretics O 664

DNA-Virusinfektionen Ka 1189

Dopamine agonists Ka 384

Dopamine antagonists Ü 937, Ü 938

Dopamine receptor Ü 1157

Dopamine-depleting drugs Ü 938

Dopergin Ka 384

Doppler echocardiography O 263

Doppler flow measurements Ka 1196

Doppler flow studies Ka 1197

Doppler sonography O 588

Doppler ultrasound Ka 1195, O 1208

Double slit technique O 166

DPI 206-106 Ü 887

Drinking behaviour, abnormal Kon 1127

Drug abuse in prison $\mathrm{O} 418$

Drug addicts 0507

Drug clearance Pharma 624

Drug therapy in the elderly Pharma 623

Drugs excreted by renal mechanisms Pharma 624

Duodenal mucosa Ka 50

Duodenal ulcer O 1059

Duplex scan Ka 1197

Duplexsonography $\mathrm{O} 830$

Durchfälle, chronische $\mathrm{O} 681$

Durstregulation Kon 1127

Dysaesthesia of the skull Ka 1195

Dysgerminomas $\mathrm{O} 12$

Dystonia Ü 935

Dystonia musculorum deformans Ü 935

E 605-forte Ka 97

Eagle effect Ka 429

Early immediate genes Ü 54

ECG Ü 744

Echocardiography O 264

Echokardiographie O 256

Eco R I EK 1084

EEG EK 136

Effector cells Ü 5

Ehrlich ascites tumor Ü 443

Eisensubstitution Ü 250

Eiweiß in Nahrung und Ernährung Bespr 190

Eiweißausscheidung O 256

Eiweißstoffwechsel O 518, O 521

Eiweißsynthese O 521

EKG Ü 745

Elastase O 901

Elastase inhibition capacity O 1054

Elastase- $\alpha 1$-proteinase inhibitor complex 01054

Electrolyte 074

Electrolyte excretion O 655

Electrolyte intake O 655

Electron probe X-ray microanalysis Ü 199

Elektrolytausscheidung O 655

Elektrolyttransportsysteme in papillären Sammelrohrzellen Ü 201

ELISA Ü 301, O 461, O 685, O 865, O 896, O 907, EK 1082

Embolic infarction of cardiac origin O 1174, O 1175, O 1176

Embolic infarctions O 1173

Embolism O 1173

Embolization Ka 1197

Embolizing deep venous thrombosis O 1208

Embryogenesis Ü 53

Emphysem Suppl XIX
Encephalitis O 921

Encephalopathy, progressive subcortical vascular O 1173

Endemic goiter O 503

Endocarditis, infective O 921

Endocrine symptoms O 669

Endocrine systems Ü 699

Endocrine treatment $\ddot{U} 347$

Endogene Natriuretische Faktoren Kon 1112

Endometriosis EK 537

Endorganschädigungen, hypertoniebedingte O 256

Endothelial cell-receptor mechanisms O 173

Endothelial cells UU 53

Endothelin O 774

Endothelin receptors O 758

${ }^{125}$ I-endothelin-1 binding sites 0758

Endotoxin Ka 979

Endstage renal disease $\mathrm{O} 65, \mathrm{O} 718, \mathrm{O} 901, \mathrm{~K}$ on 1127

End-stage renal failure $\mathrm{O} 38, \mathrm{O} 41, \mathrm{O} 709$, O 774

Endurance-training Ü 1157

Energiebilanz O 380

Enhancement of nerve regeneration EK 146

Enolase Ka 244

Enterochromaffine Zellen Ka 244

Enteroviruses Ü 789, O 914

Entscheidungstheorie Ed 297

Enzyme deficiencies in steroidogenesis O 597

Enzyme deficient patients EK 1084

Eosinophilia Ka 50, Ka 739

Eosinophilia-myalgia syndrome $\mathrm{O} 735$

Eosinophilie-Myalgie-Syndrom Ka 739

Epidemiology of hypertension $\mathrm{O} 655$

Epidermal growth factor Ü 985, Ü 986, Ü 1157

Epinephrine Ka 96

4-Epirubicin Ka 1077

Epithelial cell polarity Kon 1087

Epitheloid angiosarcoma Ka 388

Epitheloid-cell granuloma Ka 342

Epo, recombinant human-like O 405

-, replacement O 407

Epo levels O 407

Epstein-Barr virus Ü 789, O 906

Epstein-Barr-Virus-Infektionen Ka 1189

Erblindung, einseitige Ka 332

Ergometer O 476

Ergot alkaloids Ü 1163

Erkrankungen, behandlungsbedürftige Dunkelziffer Suppl XIX

Ernährungslehre Bespr 525

Erosive duodenitis O 1059

Erosive gastritis O 1059

Erregerdiagnostik bei Pneumonien O 372

Erythema migrans $\mathrm{Ka} 431$

Erythrocytapheresis O 18, O 19, O 20

Erythrocyte agglutination test according to Largo O 1020

Erythrocyte aggregation O 559

Erythrocyte antibody O 1050

Erythrocyte aspartate O 184

Erythrocyte aspartate aminotransferase O 183

Erythrocyte deformability O 559

Erythrocyte preparation O 184

Erythrocyte velocity $\mathrm{O} 173$

Erythrocytes O 23, O 559

Erythrocytic hypoplasia O 403

Erythropoetin Bespr 289

Erythropoiesis O 403

-, scheme of the regulation O 406

Erythropoietin O 403 
Erythrozytensubstitution Ka 333

Erythrozytenzerfall Ü 248

Esophageal dysfunction $\mathrm{O} 602$

Esophageal hypermotility Ka 187

Esophageal motility O 602

Esophageal sphincter, lower O 602

Esophageal strictures Ka 187

Esophago-gastro-duodenoscopy O 1059

Esophagus Ka 187

-, deep mucous glands Ka 187

Essential adrenal tumors O 320

Essential hypertension O 71, O 75, O 256, O 269, O 476

Essentielle Hypertonie O 476

Estracyt Ü 347

Estradurin Ü 347

Estramustinphosphat $\ddot{U} 349$

Estrogen Ü 1157

Estrogen bone effects Ü 359

Estrogens Ü 1160

Ethanol/tween O 173

Ethylparathion $\mathrm{Ka} 617$

Etoposide Ü 539, Ü 542

Euthyroid metabolic state O 557

Euthyroidism O 942, O 1037

Evaluierung diagnostischer Maßnahmen Ed 297

Evoked nociceptive activity EK 129

Exercise O 476, O 823

Exocrine pancreatic output $\mathrm{O} 60$

Exotoxin A Ka 525

Experimental pharmacology Bespr 182

Extracellular proteins 55

Extrusion of chemotherapeutic agents, carrier-mediated Ü 443

Factor VIII O 1186

Factor VIII: C inhibitors Ka 1071

Familial dysbetalipoproteinemia O 65

Farlutal Ü 347

Fasciitis Ka 335

Fast protein liquid chromatography O 1013

Fasziitis, granulomatöse Ka 340

Fat-soluble drugs Pharma 624

Fc receptors Ü 301, Kon 1138

Feedback Regulation, IGF Wachstumshormon Ü 992

Female-to-male transmission of HIV O 1205

Femoral vein Ka 619

Fenthion Ka 616

Fernvisus Ka 333

Ferritin O 33

Fettzellen, IGF-Wirkung Ü 991

Fetus Ü 1159

Fever Ka 525

Fever/urticaria O 1185

Fibrillation, nonvalvular atrial O 1173

Fibrin O 518

Fibrin monomer O 1020

Fibrinogen O 559, O 1186

Fibrinolyse $\mathrm{O} 1214$

Fibroblasts Ü 53, O 814

Fibronectin O 559

Fibrosis, secondary interstitial O 166

Fieber O 89

Flecainid $\mathrm{O} 1178$

Flow cytometry O 393, O 408

Flow volume measurement O 263

Fluorescein dilaurate $\mathrm{O} 60$

Fluorescence microscopy $\ddot{U} 443$

Fluoreszierende Indikatoren Ü 200
Flutamid Ü 347

Foam cells $\mathrm{O} 68$

Fosfestrol Ü 349

Fourier transform Ü 744

Fractional sodium excretion O 545

Fragmin $^{\circledR}$ EK 294

Framingham Study O 583

6 French Endosonographiekatheter O 570

French venous sheath catheter $\mathrm{Ka} 620$

Fugerel Ü 347

Fungi $O 512$

Furosemide O 545, O 869

Fused giant cells Kon 1087

Gallbladder lithiasis O 496

Gallstone analysis O 497

Gallstone structure O 496

Gammaglobulin synthesis Ka 95

Gammopathy $\mathrm{Ka} 95$

Gangrene O 207

Gastric acid O 961

Gastric antacids O 692

Gastric carcinoma O 1059

Gastric motility O 959

Gastric ulcer O 41, O 1059

Gastritis EK 488

-, atrophic Pharma 624

Gastroesophageal reflux Ka 187

Gastrointestinal bleeding Ka 290

Gastrointestinal complications in cardiac surgery O 1059

Gastrointestinal side effects of Azathioprine Ka 50

Gastrointestinal tract $\ddot{U} 702$

Gastrointestinale Endoskopie Bespr 926

Gaucher's disease $\mathrm{Ka} 94$

GBM-fragments Kon 1145

Gemfibrozil absorption O 692

Gemfibrozil elimination O 692

Generalized cytotoxicity Ka 237

Generalized likelihood ratio technique $\mathrm{O} 43$

Genes Ü 985

Genesis of ulcer O 1059

Genetische Beratung Bespr 1182

Geriatric clinical pharmacology Pharma 623

Geriatric hospital O 1175

Geriatric patients O 421

Gerinnungsaktivierung Ka 281

Gerinnungssystem O 521

Gerontology Bespr 1194

Gerontopsychiatrie Pharma 1085

Geschlechtsverkehr Pharma 582

Gestational hypertension O 559

Gewebeproliferationen Ka 1189

Gewichtsreduktion O 661

Gewichtsverlust O 89

Gewichtszunahme O 661

Giant cell myocarditis $\mathrm{Ka} 880$

Glial cells UU 53

Glioblastoma O 12, O 16

Glococerebrosidase $\mathrm{Ka} 95$

Glomerular diseases Kon 1145

Glomerular filtration $\mathrm{Ka} 878$

Glomerular filtration rate O 166, O 172, O 223, O 835

Glomerular immune functions Kon 1138

Glomerular injury Kon 1146

Glomerular mesangial cells Kon 1134

glomeruläre Filtrationsrate O 256, O 750

Glomeruli O 758 
Glomerulonephritis Ka 1190, Ka 1198, Ka 1199

-, poststreptococcal Ka 1200, Ka 1201

Glomerulus Kon 1138, Kon 1145

Glucagon $\mathrm{O} 750$

Glucagon receptors $\ddot{U} 444, \ddot{U} 887$

Glucocerebroside Ka 95

Glucocorticoid therapy O 1221

Glucocorticoids O 1217

Glucose O 228, Kon 1091

Glucose contraregulating hormones O 951

Glucose reabsorption $\mathrm{O} 233$

Glucose sensor Ü 306

Glucose-controlled insulin infusion $\mathrm{O} 951$

Glukoseoxidation O 380

Glukoseutilisation O 380

Glutamine Kon 1096

$\tau$-Glutamyl-Transpeptidase O 1009

Glutathione Ü 443, O 1008

Glutathione release into plasma O 1011

Glutathione-S-transferase Ü 443

Glycerolipids Ü 54

Glycerophosphorylcholine Ü 199, Kon 1091

Glycogen synthase Ü 54

Glycoprotein P170 Ü 443

Glycoproteins Ü 53, Preis-Vorlsg. 149

Glycosphingolipids Preis-Vorlsg. 149

Glykogen O 1179

Glykoproteine Ü 199

Gonadotropin-releasing hormon analogues EK 537

Gout Ka 874

G-protein Ü 887

Graft acceptance 0869

Graft loss O 869

Graft rejection Ü 56

Graftversus-host disease Ü 841

Granularität Kon 1106

$\alpha$-granule proteins 0847

Granulocytopenia Ü 247

Granulomatous giant cell myocarditis Ka 880

Granulomatous myositis Ka 335

Graves' disease O 942, O 1037

-, psychological disturbances O 942

Gross hemolysis O 1187

Group A beta-hemolytic streptococci Ka 427

Group A streptococci Ka 1199

-, sexual transmission $\mathrm{Ka} 524$

Group education $\mathrm{O} 77$

Growth Ü 985

Growth disorder Ü 985

Growth Factor Bespr 1065

Growth hormone O 583, O 951, Ü 985

Growth regulation Ü 53

Gruppenschulung $\mathrm{O} 77$

Guanin-Nukleotid-bindende Proteine Ü 889

Guanosine $3^{\prime}: 5^{\prime}$-cyclic monophosphate O 213, O 709

Guanosintriphosphat Ka 927

Guanylate cyclase O 213

Guillain-Barré syndrome O 1183, O 1185

Gut Ü 489, Ü 699

Gynäkomastie Pharma 581

Haemoccult ${ }^{\circledR}$-test 083,087

Haemodialyse $\mathrm{O} 827$

Haemofiltration $\mathrm{O} 827$

Hairy-Leukoplakie O 865

Haloperidol Ü 938

Hämodialyse Ka 1190
Hämodialyseverfahren, unterschiedliche $\mathrm{O} 806$

Hämodynamik Bespr 1216

Hämoglobin Ü 248

Hämolyse Ü 247

-, chronische intravasale $\ddot{U} 248$

hämolytische Krise Ü 251

Hämosiderin Ü 248

Hämosiderin-Nachweis Ü 250

Ham-Test Ü 249

Hancock prostheses O 263

Harnwegsinfekte O 1046

Haut-Faszie-Muskel-Biopsie Ka 341

Hautfibrome O 83, O 87

Hautkrankheiten Bespr 487

HBV O 507

HDL O 65

HDL-cholesterol O 65

Headache O 1185

Heart Bespr 536, Ü 699

Heart failure O 709, O 797, Ü 887, O 921

Heart rate $\mathrm{O} 1168$

Heart rate variability 026

Heart transplantation O 797, O 914

Heat stress nephropathy $\mathrm{Ka} 874$

Heavy beer drinking $\mathrm{Ka} 878$

Hemangioendothelioma Ka 388

Hematocrit O 18, O 559

Hematological malignancies O 372

Hematopoietic cells Ü 53

Hematopoietic stem cells Ü 539

Hematuria Ka 1200

Hemodialysis O 41, O 65, O 183, O 269, O 774, O 806, O 901, Kon 1127

Hemodynamics O 559

Hemoglobin O 18, O 403

Hemoglobinuria, paroxysmal nocturnal Ü 247

Hemolysis, chronic intravascular Ü 247

Hemolytic anemia $\mathrm{Ka} 95$

Hemolytic group A Ka 523

Hemophilia O 1203

Hemorrhage, intracranial O 1173

Hemorrheology O 559

Henle'sche Schleife Kon 1110

Heparin O 1184

Hepatic damage O 1040

Hepatic failure Ka 525, Ü 841

Hepatic nitrogen metabolism Kon 1096

Hepatic triglyceride lipase $\mathrm{Ka} 526$

Hepatic ureogenesis Kon 1096

Hepatitis Ka 237

Hepatitis A O 415

Hepatitis A virus 0906

Hepatitis B O 415, EK 1082

Hepatitis B virus O 906

Hepatitis C Ka 1032, EK 1082

Hepatoblastom Ü 988

Hepatocellular carcinomas $\ddot{U} 443$

Hepatocyte heterogeneity Kon 1096

Hepatoma Ü 443

Hepatopathie Ka 1189

Heroin abusers O 507

Herpes esophagitis Ka 187

Herpes genitalis O 865

Herpes simplex virus O 906, O 1003

Herpes-simplex encephalitis $\mathrm{Ka} 286$

Herpes-simplex-Virus Ka 1189 , Ka 1190

Herzfrequenz O 825 
Herzglykose Ü 893

Herzinsuffizienz Ü 893

Herzoperationen, Veränderungen des oberen Gastrointestinaltraktes O 1059

Herzrhythmusstörungen $\ddot{U} 744$

Herztod, plötzlicher Ü 744

Herzzeitvolumen O 1179 , O 1180

Herzzeitvolumenindex O 1179

Heterogeneity O 223

Heterosexual transmission of HIV O 1203

Hiatal hernias Ka 187

High erythrocyte sedimentation rate $\mathrm{Ka} 881$

High-density lipoproteins O 65

High-risk keratoplasty 0869

Hind III EK 1084

Hirnerkrankung, vaskuläre Bespr 453

Hirnmetastasenoperation O 576

-, Kontraindikationen O 579

Hirsutism O 597

Hirsutismus O 597

Histamine Ü 887

Histamine receptors Ü 887

Histidine-tryptophan-ketoglurate solution O 223

HIV Ü 299, O 393, O 507, O 853, Ka 1032, O 1050, EK 1082, O 1203, EK 1229

-, Africa O 1206

-, risk factors O 415

-, seroepidemiological features $\mathrm{O} 415$

-, virus isolations O 1204

HIV antibodies Ka 237, O 906

HIV antibody testing $\mathrm{O} 419$

HIV infection O 43, O 397, O 415, O 906

HIV positive patients $\mathrm{O} 14$

HIV transmission O 418

HIV-antigen Ka 237, O 864

HIV-encephalitis O 12

HIV-infection O 89

HIV-Infektion, Stadieneinteilungen Ka 92

HIV-positive partners O 1204

HIV-1 infection, early stages O 393

$\mathrm{HIV}-1$ virus $\mathrm{O} 43$

HIV-1-Antigenämie 0864

HIV-1-Antigenemia O 864

HLP O 65

Hodenkarzinom O 865

Hodgkin's disease Ü 539, O 847, Bespr 905

-, bone marrow transplantation UU 539

-, chemotherapy Ü 539

Homogeneous cell populations from the kidney Ü 199

Homosexual men O 43, EK 1082

Homosexuals O 419, O 906

Honvan Ü 347

Hormone measurement O 405

Hormone resistant tumor Ü 347

Hormone treatment Kon 1091

Hormone-related malignant tumors Bespr 1194

Hormones O 814

Hormontherapie Ü 347

-, adjuvante primäre Ü 348

-, palliative primäre UU 348

-, sekundäre Ü 349

-, sofortige oder verzögerte Ü 349

Hot and dry climate $\mathrm{Ka} 879$

24-hour blood pressure measurements O 768

Human fetal and adult kidney 0758

Human heart O 797

Human immunodeficiency virus O 89, Ka 237, O 864
-, heterosexual transmission O 1203

Human insulin O 447

Human mononuclear leukocytes $\mathrm{O} 71, \mathrm{O} 74$

Human myocardium Ü 887

Human-herpesvirus $6 \quad \ddot{U} 789$

Humidifier Lung O 512

Hybridization techniques O 1003

Hydrocortison Ka 928, Ka 929

Hydronephrotic kidney O 166

18-hydroxycorticosterone Ka 981

Hydroxyethylstarch O 559

21-Hydroxylase-Defekt Ka 330

21-Hydroxylase-deficiency O 597

3-hydroxy-methylglutaryl coenzyme A reductase O 814

17-Hydroxyprogesteron Ka 329

$3 \beta$-Hydroxysteroiddehydrogenase deficiency O 597

25-hydroxyvitamin D O 421

3-hydroxy-3-methyl glutaryl coA reductase mRNA Ü 54

Hypalbuminämie Ka 1034

Hyperaldosteronism O $180, \mathrm{Ka} 981$

Hyperbicarbonatemia O 175, O 178

Hypercholesterolemia O 814

Hypercortisolism Ka 981

Hyperdynamic reactions O 1171

Hypergammaglobulinämie Ka 1034

Hypergammaglobulinemia O 1050

Hyperimmunoglobulinemia O 906

Hyperinsulinämie, IGF-Spiegel Ü 996

Hyperkalemia O 38

Hyperlipidemia O 454

-, therapy-refractory Ka 526

Hyperlipoproteinemia O 41, O 65

Hyperparathyroidism O 38, Ü 359, O 669

Hyperphosphatemia Ka 927

Hyperpigmentierung der Haut Ka 1076

Hyperprolactinemia Ka 384, Ü 1157, Ü 1161

Hyperprolaktinämie Ka 384, Bespr 1049

-, IGF-Spiegel Ü 997

Hypersplenism Ka 95

Hypertension Suppl XX, O 320, O 655, O 723, O 768, O 774,

O 801 , O 830 , Kon 1119, O 1171

-, arterial O 38

-, Epidemiology O 583

-, non-pharmacological therapy O 664

-, pulmonale O 1210

-, pulmonary O 38

Hypertensive acromegalics, female O 583

Hypertensive hemodialysis patients $\mathrm{O} 718$

hypertensive Nephropathie O 256

Hyperthyreose, oligosymptomatische Formen Pharma 647

-, schwere Verlaufsformen Pharma 650

Hyperthyreoseformen, Pathophysiologie Pharma 631

Hyperthyreosen Pharma 627

Hyperthyroidism EK 441, O 552, O 964, O 1013, O 1037

Hypertonia Ü 935

Hypertonie Bespr 48, Bespr 511, O 661

-, arterielle Kon 1119

-, chronische O 559

Hypertriglyceridemia O 38

Hypertrophie, myokardiale O 256

Hypertrophy, left ventricular O 256

Hyperuricemia O 1222

Hyperuricemia and gout $\mathrm{Ka} 874$

Hyperuricosuria Ka 874

Hyperventilation $\mathrm{O} 806$

Hyperviscosity syndromes O 1183

Hypocalcemia Ka 525, O 678, Ka 927 
Hypocomplementemia Ka 1200

Hypocortisolism Ka 927

Hypoglycemia O 447, O 957

Hypoglycemia unawareness $\mathrm{O} 447$

Hypokalemia Ka 981

Hypomagnesemia O 678

Hypomagnesiämie $\mathrm{O} 835$

Hyponatremia Ka 191

Hyponatriämie Ka 191

-, Notfall-Therapie Ka 195

Hypoparathyroidism O 678

Hypophospatemia O 678

Hypophysärer Minderwuchs, IGF-Spiegel Ü 994

Hypophysenadenome Ka 384

Hypopitiutarismus Ü 987

Hypoproteinemia O 1187

Hypotension O 313, O 1168, O 1184, O 1185, O 1186

Hypotension with bradycardia syndrome O 313

Hypotensive reactions O 1168

Hypothalamic control Ü 1159

Hypothalamic pituitary dysfunction O 964

Hypothalamus $\ddot{U} 1157$

Hypothalamus-Hypophysen-Achse O 968

Hypothyroidism O 964, O 1013

Hypothyroidismus, IGF-I Werte Ü 996

Hypotonia Ü 935

Hypouricemia O 1222

Hypovolaemia, postdialytic Kon 1127

Hypovolemia O 24

Hypovolemic shock O 313

Hypoxanthine Ü 444

Hypoxia O 806, O 1187

$\mathrm{H}_{2}$-receptor antagonists $\mathrm{O} 959$

ICI 176334 Ü 347

Ig A myelomatosis Ka 526

IgA-positive cells O 12, O 14, O 16

IGF Wirkung im ZNS Ü 993

IGF-binding proteins Ü 985

IGF-Bindungsprotein Ü 989

IGF-I Ü 986

IGF-I Konzentration im Serum während der Pubertät Ü 987

IGF-I RIA Ü 987

IGF-II Ü 986

IGF-Insulin Interaktion Ü 993

IGF-Rezeptoren Ü 990

IgG Ka 237

IgG antibody titers against B.b. in serum $\mathrm{Ka} 431$

IgG Levels O 686

IgG plasmacytoma O 16

IgG uptake Kon 1138

IgG-anticardiolipin antibodies O 594

IgG-positive cells O 14, O 16

IgM Ka 237

IgM antibodies $\mathrm{Ka} 431$

IgM paraproteinemia Ka 1027

IgM-anticardiolipin antibodies O 594

IgM-positive cells $\mathrm{O} 12, \mathrm{O} 16$

125-I-labeled fibrinogen test O 1020

Iliac arteries O 570

Iliac vein Ka 619

Illness behaviour $\mathrm{O} 77$

IL-6 O 685

Immune complexes Ü 299, O 1066

Immune defense mechanisms Ü 299

Immune system Ü 489, Ü 704

Immune thrombocytopenia $\mathrm{Ka} 95$
Immunglobuline, thrombozyten-assoziierte O 460, O 463

Immunhämatologie Bespr 1156

Immunkomplexe, zirkulierende 089

Immunoassays O 33 , Ka 324

Immunoblotting Kon 1138

Immunocompromised patients $\mathrm{O} 372$

Immunocytochemistry $\mathrm{O} 12$

Immunocytoma Ka 1027

Immunofluorescence O 1003

Immunoglobulin 688

Immunoglobulin A Ka 526

Immunoglobulin-containing cells $\mathrm{O} 12$

Immunohistochemistry O 1003

Immunologic monitoring O 408

Immunologic responses against tumor $\ddot{U} 8$

Immunomodulation $\ddot{\mathrm{U}} 2$

Immunomodulatory mediators $\ddot{U} 8$

Immunoperoxidase technique 0728

Immunopharmacology Bespr 1207

Immunoreactive trypsin O 565

Immunoregulatory T-cell dysfunction Ka 978

Immunosuppression Ü 306, O 408

Immunosuppressive cytostatic therapy Ka 1189

Immunosuppressive pharmacotherapy Ka 976

Immunosuppressive therapy $\mathrm{Ka} 1071$

Immunotherapy Ü 1

-, adoptive vs nonadoptive $\ddot{U} 7$

Immunsuppressionstherapie Ka 1189

Immunsuppressiva, Reduktion Ka 1193

Immunsuppressive Therapie O 408, Ka 1190

Immunsystem O 521

Immuntoleranz Bespr 773

Impairment of memory Ka 1195

Increased urine acidity $\mathrm{Ka} 878$

Indometacin O 383

INF $\alpha$ EK 1229

Infarction O 594

Infection with HIV and CMV Ka 237

Infectious Mononucleosis Bespr 886

Infective endocarditis O 921

Infektionserreger des Menschen Bespr 240

Infertility Ü 1157

Inflammation Ü 53

Inflammations, chronic O 14

Inflammatory diseases $\mathrm{O} 12$

Inflammatory joint disease $\mathrm{O} 685$

Infusionstest nach Ellsworth and Howard Ka 930

Inhibitory guanine nucleotide-binding proteins Ü 887

Innere Medizin Bespr 296, Bespr 580, Bespr 1182

Inositol Kon 1091

Inotropic stimulation Bespr 345

Insecticide $\mathrm{Ka} 616$

Inseltransplantation $\ddot{\mathrm{U}} 307$

Insulin O 447, O 951

Insulin like growth factor-Bindungsprotein Ü 985

Insulin pump treatment $\ddot{U} 306$

Insulin treatment Ü 306, Kon 1091

Insulin-dependent diabetic patients 026

Insulin-like growth factors (IGFs) Ü 985

Insulinpumpen Ü 308

Insulin-resistenter Diabetes mellitus Ü 998

Intensivmedizin Bespr 1182

Intensivpatienten, pulmonale Probleme Bespr 788

Interdigitated bilayer systems Preis-Vorlsg. 149, PreisVorlsg. 150

$\beta$-Interferon Ü 1, Ü 3, Ka 286

interferon alpha Ü 1 
Interferon beta EK 1229

Interferon gamma EK 1083, EK 1229

Interferon therapy Ka 241

Interferons $\ddot{U} 1$

Interferon-Therapie Ka 241

Interleukine Ü 985

Interleukin-1 EK 1083, O 1217

Interleukin-1 $\propto$ Ü 300

Interleukin-1 production, circadian rhythm O 1217

Interleukin-2 $\dot{\mathrm{U}} 1, \ddot{\mathrm{U}} 2, \ddot{\mathrm{U}} 3, \mathrm{O} 728$

Interleukin-2 receptor $\ddot{U} 2$

Interleukin-6 O 685, EK 1083

Internal carotid artery Ka 1195

Intersalt-Studie O 655

Interstitial osmolality Kon 1091

Intestinal complaints $\mathrm{O} 83$

Intoxikation Bespr 1182

Intraarterial digital subtraction angiography Ka 1197

Intracellular calcium Kon 1112

Intracellular electrolytes $\mathrm{O} 71$

Intracellular $\mathrm{pH}$ Kon 1088

Intracranial hemorrhage O 1174

Intracranial pressure monitoring Ka 286

Intragastric $\mathrm{pH} \quad \mathrm{O} 959$

Intraocular B-cell lymphoma Ka 1027

Intraoperative endoscopical diaphany $\mathrm{Ka} 290$

Intrasellar tumor Ka 342

Intratubular obstruction $\mathrm{Ka} 874$

Intravascular coagulation syndrome O 1174

Intravascular sclerosing bronchioloalveolar tumor Ka 388

Intravascular ultrasound $\mathrm{O} 570$

Intravenöser Drogenabusus Ka 1034

Intravenous drug abusers $\mathrm{O} 415$

Inulin $\mathrm{O} 750$

Iodine O 1039

lodine deficiency $\mathrm{O} 503$

Iodine prophylaxis O 503

Ion transporters Kon 1087

Iron $\mathrm{O} 41, \mathrm{O} 507$

Iron deficiency $\mathrm{O} 23$

Iron therapy $\mathrm{O} 23$

Ischämie O 383

Ischämische Schädigung O 1179

Ischemia O 223, O 225

Ischemic heart disease o 518

Ischemic lesions O 1173

Isoamylase $\mathrm{O} 565$

Isotope, stabile O 521

Isovolumetrische Messungen O 1178

Isozymes Kon 1134

Isradipine O 797

Ivermectin Bespr 926

Ixodes ricinus ticks $\mathrm{Ka} 431$

Joint pain $\mathrm{O} 466$

$\mathbf{K}^{+}$channels Kon 1087

Kalium O 678

Kallikrein-Kinin-System O 380

Kalzitonin Ka 244

Kalzium Ka 929

Kammerflimmern Ü 744

Kammertachykardien Ü 744

Kaposi's sarcoma Ü 301, O 1050, EK 1229

Kaposisarkom O 865

Kardiodepressive Nebenwirkungen O 1178

Kardiodepressive Wirkung 01178
Kariovaskuläre Erkrankungen O 662

Katecholamine Ü 893

Kernresonanzspektroskopie Ü 200

Ketoconazole Ü 354, O 869

Ketosteroide im 24 h-Urin Ka 328

Kidney O 223, Ka 1032, O 1042, Kon 1087, Kon 1091, Kon 1138

Kidney development 0758

Kidney diseases O 41, Ü 985

Kidney disorders $\mathrm{O} 923$

Kidney epithelial cells Ü 53

Kidney transplantation Ü 306, O 774, Ka 1189

Kidneys, ${ }^{125}$ I-endothelin binding sites $\mathrm{O} 758$

-, enlarged Ka 1198

Klasse-I Antiarrhythmika O 1178

Klinisches Wörterbuch Bespr 190

Klinisch-pharmakologische Datensammlung Bespr 475

Knochendichte Ü 364

Knochenfestigkeit Ü 364

Knochenmark O 521

Knochenmarksinsuffizienz Ka 333

Knochenmarktransplantation Ü 252

Knochenstoffwechsel Ü 362

Knochenstruktur Ü 364

Knorpel, IGF-Wirkung Ü 992

Kochsalzreiche Diät O 827

Kognitive Störungen O 969

Kolonkarzinom Ü 988

Kolonneoplasien O 83, O 87

Komplement-Komponenten Ü 247

Komplement-regulierendes Protein Ü 247

Kompressionsbehandlung O 1213

Kontraktionskraft des Herzens, Regulation Ü 888

Koronardilatation $\mathrm{O} 823$

Körpergewicht, relatives O 655, O 661

Kortikosteroid-Behandlung Ka 340

Krankheitsverarbeitung O 77

Kreatinin O 678

Kreatininausscheidung O 661

Kreatinin-Werte Ka 1191

Krebs Bespr 1177

Krebsforschung Bespr 1156

Kreislauf, kleiner O 823

Künstliches Pankreas Ü 308

kutane Anergie O 89

Lack of nocturnal blood decrease O 768

Lactate dehydrogenase O 397

Lactic dehydrogenase $\mathrm{O} 65$

Lactoferrin O 901, O 903

Lake-tahoe-disease Ü 789

Lambert-Eaton myasthenic syndrome O 1188

Langzeit-EKG O 748

Langzeit-Elektrokardiographie Bespr 1201

Large infarction O 1174

Lasronscher Zwergwuchs, IGF-I und -II Spiegel Ü 995

Late potentials $\dot{U} 744$

LDH O 65

LDL O 65

LDL-cholesterol O 65, O 454, O 664

LDL-lipoproteins O 1183

Lebenserwartung $\mathrm{O} 662$

Leber O 521

Leberstoffwechsel O 521

Leberzirrhose, IGF-I Spiegel Ü 995

Lecithin-cholesterol acyltransferase O 269

Lectin-induced cytotoxicity O 896 
Left ventricular hypertrophy O 256

Left ventricular outflow tract O 264

Legionella O 372

Leiomyom Ü 988

Leiomyosarkom Ü 988

Lektine Ü 199

Lendenwirbelsäule, akute Beschwerden EK 116

Lendenwirbelsäulensyndrome EK 107

Leprosy Ü 493

Leucocytosis Ka 739

Leukapheresis techniques $\ddot{U} 1$

Leukemia O 403

Leuko-araiosis O 1176

Leukocytes O 559, O 901

-, polymorphonuclear Ka 1199

Leukocytosis O 901, Ka 976

Leukopenia O 901, O 1050

Leukopenie O 89

Leukotrienes $\mathrm{O} 172$

Levodopa blood levels Pharma 623

Levodopa medication Ü 935

LH RH analogs Ü 347

LH-Produktion, Suppression Ü 351

Lichtreflexionsrheographie O 1208

Lidocaine Ka 286, O 673

Life satisfaction $\mathrm{O} 77$

Linksventrikuläre Funktion O 1181

Lipase O 60, O 63, O 565

Lipid abnormalities O 65

Lipid bilayer Preis-Vorlsg. 149

Lipid guidelines $\mathrm{O} 454$

Lipid levels $\mathrm{O} 454$

Lipid testing O 459

Lipid-lowering concept $\mathrm{O} 65$

Lipids O 271, O 664

Lipidstoffwechsel O 521

Lipopolysaccharide O 1217

Lipoproteins O 269, O 271, O 814

Liposarkom Ü 988

Lisurid Ka 384

-, Nebenwirkungen Ka 386

Lisuride $\mathrm{Ka} 384$

Lithium O 223, O 507, O 1039

Lithium Transport O 232

Liver Kon 1096

Liver cirrhosis O 175, O 180, O 545, O 692, O 709, O 774

Liver disease O 692, Ü 985

Liver mass, decline Pharma 623

Lobar cerebral hemorrhage O 1174

Locked-in-syndrome Ka 191

Loss of vision $\mathrm{Ka} 1027$

Low density lipoprotein O 65, O 454, O 664

Low density lipoprotein receptor mRNA Ü 54

Low density lipoprotein-cholesterol O 814

Low light level in vitro monitoring $\mathrm{O} 33$

Low molecular weight heparin EK 294

Low output heart failure syndromes 0313

Low-frequency component O 26

L-thyroxine O 1040

L-Tryptophan O 735

L-tryptophane Ka 739

Luciferin-luciferase reactions $\mathrm{O} 35$

Lues O 415

Luftbefeuchter O 512

Luftfilter O 516

Lumbago EK 107, EK 116

Luminescence immunoassays O $33, \mathrm{O} 35$
Luminol-peroxide-4-iodophenol reaction O 33

Luminometrie Ü 200

Lung cancer $\mathrm{O} 728$

Lung disease $\mathrm{O} 857$

Lung function $\mathrm{O} 857$

Lung infiltration Ka 1198

Lungenbiopsie O 372

Lungenembolierisiko O 1213

Lungenfunktionsprüfung $\mathrm{O} 514$

Lungs Ü 699, Ü 700

Lupus anticoagulant O 594

Lupus erythematodes O 774

Lupus erythematosus Ü $300, \mathrm{O} 460$

Luteinisierendes Hormon $\mathrm{Ka} 927$

Lyme borreliosis $\mathrm{Ka} 431$

Lyme carditis $\mathrm{Ka} 431$

Lymphadenopathie O 89, Ka 1189

Lymphadenopathie Syndrom O 89

Lymphadenopathy syndrome O 906

Lymphocyte Homing Bespr 212

Lymphocyte subpopulations O 942

Lymphocytes O 71, Bespr 217, O 408, O 896

-, adoptive transfer $\ddot{U} 2$

Lymphocytic meningoradiculitis Bannwarth $\mathrm{Ka} 431$

Lymphoid tissue Ü 703

Lymphokine-activated killer cells $\ddot{U} 1$, Ü 3

Lymphokine-induced cellular toxicity Ü 5

Lymphokines Ư 489

Lymphomas O 12

Lymphomatous leptomeningitis Ka 1076

Lymphome, maligne Ka 1189

-, Rückbildung Ka 1193

-, solitäre intrazerebrale Ka 1190

-, zerebrale Ka 1192

Lymphopenie O 89

Lymphoplasmocytoid lymphomas O 16

Lymphoreticular disorders $\mathrm{Ka} 95$

Lymphozytensubpopulationen O 408, O 410, O 853

Lyso-phosphatidylethanolamine EK 49

Lysozym Ka 340

Lysozyme Ka 335

Macobacterial antigens $\ddot{U} 489$

Macrophages O 218

Macroprolactinemia Ü 1157

Macrovascular hyperemic response of blood flow O 588

Maculopapules Ka 1200

Madin-Darby canine kidney cells Kon 1087

Magnesium O 223, O 507, O 835

Magnesium Excretion O 228

Magnesium therapy $\mathrm{O} 678$

Magnesium Transport O 233

Magnesiumausscheidung $\mathrm{O} 835$

Magnetic resonance Ka 1196

Magnetic resonance imaging Bespr 396

Makrophagen $\mathrm{O} 853$

Makroprolaktinome Ka 384

Malaria chemoprophylaxis O 277

Malaria tropica Ka 281

Malignant lymphomas Ka 874, Ka 1027

Malignant melanoma Ü 1

Malignant non-Hodgkin-lymphoma Ka 1189

Malignant tumors $\mathrm{O} 38$

Malnutrition Ka 191, Ü 985

Malonyldialdehyde O 460

Mamma-Karzinosarkom Ü 988

Mangelernährung, IGF-I Spiegel Ü 997 
Mb. Osler Ka 290

MDCK cells Kon 1087

Mediastinal parathyroid adenoma $\mathrm{O} 472$

Medical history taking O 466

Medikamenteneinwirkung auf den Sexualpartner Pharma 581

Medrogeston Ü 354

Medroxyprogesteronazetat Ü 352

Medtronic Hall prostheses O 263

Medullary capillaries O 758

Medullary thyroid carcinoma O 669

Mefloquine O 277

Megakaryocytes Ü 53

Megakaryozyten O 521

Megestat Ü 347

Megestrolazetat Ü 352

Melphalan Ü 542

Membrandefekte Ü 247

Membrane activities Preis-Vorlsg. 150

Membrane defects O 75, Ü 247

Membrane proteins UU 247

Membranenzyme Ü 199

Membrane-related neutrophil dysfunctions O 901

MEN O 669

Meningeal neoplasm Ka 1076

Meningeosis lymphomatosa $\mathrm{Ka} 332$

Meningiosis lymphoblastica Ka 1076, Ka 1080

Meningitis, proteinase antiproteinase Imbalance O 1054

Meningococcal sepsis Ka 976

Meningopolyneuritis Bannwarth O 12

Menopause O 661

Mesangial cells Ü 53, Kon 1134, Kon 1138, Kon 1145, Ka 1199

Mesangium O 835, Kon 1145

Mesenchymal cells Ü 53

Messenger ribonucleic acid Ü 699, Ü 1157

Metabolic alkalosis O 178, O 180

Metabolic defect Ka 526

Metabolic disorders O 1183

Metabolic effects O 380

Metabolic pathways in isolated cells Ü 199

Metalloproteinases Kon 1145

Metastasen in den Nebennieren Ka 1080

Metastatic carcinoid Ka 241

Metastatic neoplasms Ü 1

Methimazole therapy O 1040

Methotrexat Ka 333

Methotrexate Ü 443

Methylparathion $\mathrm{Ka} 617$

Methylprednisolone O 1217

Methylprednison Ka 1189, Ka 1193

5-Methyltetrahydrofolate $\ddot{\mathrm{U}} 444$

Metoclopramid O 607

Mexiletine $\mathrm{O} 673$

Micelles O 60

Microalbuminuria O 750

Microproteinuria O 256

Microvessel diameters $\mathrm{O} 173$

Mid-frequency component O 26

Mikroalbuminurie O 256

Mikrodissektion Ü 199

$\beta_{2}$-Mikroglobulin Kon 1150

Milrinone Ü 887

Mineralocorticoid effector mechanism O 75

Mineralocorticoid hormone Kon 1089

Mineralocorticoid receptors O 71, O 75

Mineralocorticoids O 74

Miscellaneous tissues Ü 703
Misoprostol O 961

Mistletoe lectin O 896

Mitochondrial enzyme P450 C11 Ka 981

Mitogen-Stimulierbarkeit der Lymphozyten O 89

Mitral valve disease 0921

Mitral valve prosthesis O 264

Mitral valve replacement-operation 0797

Mixed-chain phosphatidylcholines Preis-Vorlsg. 151, PreisVorlsg. 158, Preis-Vorlsg. 160

Mixed-chain phospholipids Preis-Vorlsg. 149, PreisVorlsg. 150

Molsidomine O 213

Monitoring cardiac glycoside levels Ka 327

Monoclonal antibodies O 408, O 1003

Monocytes Ü 53, O 1217

Monocytes/macrophages Ü 53

Monoglycerides O 63

Monoklonale Antikörper Ü 199

Mood states O 552

Morbidity of patients after renal transplantation $\mathrm{O} 38$

Morbus Addison Ka 328

Morbus Basedow Pharma 637

Morbus Rendu-Osler-Weber Ka 290

Morphine/Paracetamol, combined administration EK 129

3-morpholino-sydnonimine O 213

Motilitätsstörung O 603

MRI Bespr 435

mRNA Kon 1138

MR-Spektroskopie Bespr 757

M-Typing Ka 427

Mucinous cystadenocarcinoma $\mathrm{Ka} 780$

Mucinous cystadenoma $\mathrm{Ka} 780$

Müdigkeitssyndrom Ü 789

Multidrug resistance Ü 443

Multimetastasierung O 579

Multiple endocrine adenomatosis Ü 1157

Multiple endocrine neoplasia O 669

Multiple hormone resistance $\mathrm{Ka} 927$

Multiple myeloma Ka 95, Ka 526

Multiple sclerosis O 12 , O 1183

Münchhausen-Syndrom Bespr 198

Mundsoor O 865

Munich Blood Pressure Study O 583

Muscle Pharma 623

Muscle enzyme defects EK 1084

Muscle Na-K ATPase Pharma 624

Muscle oxygen pressure O 211

Muscular imaging $\mathrm{Ka} 335$

Muskelkontrakturen Ka 340

Muskelzellen, IGF-Wirkung Ü 991

Myalgia Ka 739

Myalgic encephalomyelitis Ü 789

Myasthenia gravis O 1183, O 1185

Mycobacteriosis O 1050

Myelinolyse, zentrale pontine Ka 191

Myeloablative chemotherapy Ü 539

Myeloproliferative disorders O 847

Myoadenylate deaminase gene EK 1084

Myocardial contractility O 1178

Myocardial energetics Bespr 345

Myocardial infarction O 41, O 313, O 594, Ü 744, EK 1083 , O 1187

Myocardial involvement in Wegener's granulomatosis Ka 884

Myocardial uptake of antiarrhythmic drugs O 673

Myocardium O 797

Myogenic responses O 173

Myoinositol Ü 199 
Myokard O 1178

Myokardfunktion O 1180

-, kontraktile O 1178

Myokardiale Aufnahme von Lidocasin, Mexiletin und Amiodaron $\mathrm{O} 673$

Myokardinfarkt O 521

Myopathy O 735

Myopericarditis $\mathrm{Ka} 431$

Myositiden, granulomatöse Ka 335

Myositis Ka 237

$\mathbf{N}$-acetyl- $\beta$-D-glucosaminidase $\mathrm{O} 869$

$\mathrm{Na}^{+}$-channel activators Ü 887

Nachtschweiß O 89

$\mathrm{Na}^{+} / \mathrm{H}^{+}$antiport activation $\mathrm{U} 54$

$\mathrm{Na}^{+} / \mathrm{H}^{+}$exchange Kon 1087

Nahrungsproteine Bespr 392

Naloxone hydrochloride Ka 615

Narcosis O 1168

Natrium O 661, Ü 893

Natrium-Kalium-ATPase Ü 893

Natrium-Rückresorption O 682

Natriuretic hormone Kon 1112

Natural killer Ü 3

Natural killer cell activity O 393

Natural killer cells O 1205

Nausea Ka 50

Nausea/vomiting O 1185

Near-normoglycemia Ü 306

Nebennieren Ka 1076

Nebennierenrindeninsuffizienz Ka 927, Ka 928

Nebennierenrindenstimulation Ka 328

Nebennierentumoren Ka 328

Necrotizing glomerulonephritis Ka 880

Necrotizing vasculitis Ka 437

Necrotizing vasculitis of the lungs $\mathrm{Ka} 880$

Needle sharing O 418

Negative inotropism O 1178

Negative-inotropic effects O 1178

Negativ-inotrope Wirkung O 1180

Neoplasms Ü 1

Neopterin O 43, O 218, O 864

Nephritis Ka 237, Ka 881, Ka 1198

Nephron-collecting-duct system Kon 1102

Nephron-Sammelrohrsystem Kon 11107

Nephropathie, diabetische O 257

Nephropathy O 256

Nephroprotection O 835

Nephrotic syndrome Ka 976

Nephrotoxicity O 166, O 869

Nerve growth factors Ü 986

Nervenregeneration Ü 998

Neues von klinischer Relevanz Suppl XIX

Neuroblastom Ü 988

Neurogliazellen, IGF-Wirkung Ü 992

Neurologic examination Ka 615

Neurological diseases O 1013, O 1183

Neurological examination O 589

Neurological symptoms O 1174

Neurologie Bespr 580, Bespr 1182

Neuromuskuläre Krankheiten Bespr 345

Neuropathy O 588, O 735, O 1168

-, autonomic O 26

-, peripheral O 26

Neuropsychological tests O 552

Neurotransmitter Pharma 1085

Neutral lipids O 60
Neutropenia O 903

Neutrophil degranulation O 901

Neutrophil-derived elastase O 903

Neutrophils O 901, Kon 1145

$\mathrm{NH}_{4}^{+}$excretion $\mathrm{O} 175$

Nichtenzymatische Glykosylierung von Proteinen Bespr 102

Nierenerkrankungen, IGF-Spiegel Ü 995

Nierenfunktion, Untersuchung Ü 199

Niereninsuffizienz Ka 100, O 256, O 1042

-, terminale Kon 1127

Nieren-Leber-Transplantationen Ka 1193

Nierenschrumpfung Ka 1190

Nierentransplantation O 408, Ka 1189, Ka 1190

Nierentumor Ka 1034

Nierenzellkarzinome, Klassifikation Kon 1102

Nierenzelltumoren Kon 1110

Nierenzyste Ka 1190

Nifedipine Ka 189, O 797

Nilutamid Ü 347

Nitrates Pharma 624

Nitrendipine O 166, O 758, O 797

Nizoral Ü 347

NMR Bespr 396

Nocturnal hypertension Kon 1119

Non small cell lung cancer $\mathrm{O} 728$

NonA-non B hepatitis O 1187

Nonbacterial thrombotic endocarditis O 1174

Non-chloride sodium salt O 664

Non-dihydropyridines O 797

Non-embolic infarction of cardiac origin O 1175

Non-ergot drugs $\ddot{U} 1163$

Non-hemophiliacs Ka 1071

Non-Hodgkin-Lymphom Ka 1189, Ka 1190, Ka 1191, Ka 1193

Non-Hodgkins lymphoma Ka 332, Ka 1027, O 1050, Ka 1076

Nonrenal anemic patients $\mathrm{O} 403$

Non-responders to $\mathrm{H}_{2}$-receptor antagonists $\mathrm{O} 959$

Nonsmokers O 512

Noradrenaline Ü 887

Noradrenaline administration via microdosing pump EK 873

Noradrenergic inhibition O 269

Norcpinephrine Ka 96, Kon 1127

Norfenefrin-Therapie Ka 99

Normoglykämic Ü 306

Normotensive hemodialysis patients 0718

Normotensive pregnancy O 768

Northern blot analysis Kon 1138

${ }^{15} \mathrm{~N}$ Tracertechnical investigations $\mathrm{O} 518$

${ }^{15} \mathrm{~N}$-Tracertechnische Untersuchungen $\mathrm{O} 518$

Nuclear magnetic resonance Ka 191

Nucleic acid hybridization O 914

Nutcracker esophagus Ka 187

Nutritional advice $\mathrm{O} 458$

Nutritional deficiency $\mathrm{O} 510$

Obesity O 655

Objective response of interferon therapy Ka 241

Occlusion of the iliac vein $\mathrm{Ka} 619$

Occult bleeding $\mathrm{O} 41$

Occult blood loss O 23

Oesophagusmotilität O 602

Oesophagusphinkter O 605

Oesophagusstriktur O 602

Oestrogene application, transdermal EK 537

Office blood pressure $\mathrm{O} 723$

Ofloxazine Ka 1032

Oligouria Ka 51 
Oncocytomas Kon 1102

Ophthalmological surgery O 1168

Opioids Ü 1157

Opportunistic infections $\mathrm{O} 372$

Oral food intake O 565

Oral pancreatic function test $\mathrm{O} 60$

orale mukokutane Herpes-Infektion O 865

Orchiectomy Ü 347

Organ protection $\mathrm{O} 223$

Organ transplantation O 869

Organic osmolytes Ü 199, Kon 1091

Organophosphate poisoning $\mathrm{Ka} 96, \mathrm{Ka} 615$

Organophosphorus compounds $\mathrm{Ka} 615$

Orimeten Ü 347

Orthostatic load O 26

Osmodiuretics Ka 286

Osmoregulation Ü 199

Osmoregulation in papillären Sammelrohrzellen Ü 202

Osteoarthritis O 685

Osteoclast inhibiting effect Ü 359

Osteoporose Ü 361

Osteoporosis Ü 359

Östradiol Pharma 581

Östrogen Pharma 581, Ü 988

Ouabain-ähnliche Faktoren Kon 1112

Ouabain-like factor Kon 1112

Ovarian tumor Ka 342

Oxygen histograms O 207

Oxygen radicals $\ddot{U} 443$

P. falciparum malaria Ka 281

Paget's disease Ü 359

Pancreas Ka 780

-. human O 60

Pancreas transplantation $\ddot{U} 306$

Pancreatic cancer Ka 780

Pancreatic enzyme levels O 565

Pancreatic function $\mathrm{Ka} 780$

Pancreatic isoamylase $\mathrm{O} 565$

Pancreatitis, edematous O 565

Pancreolauryl test O 60, O 63

Pancytopenia Ka 237

Panik Bespr 1202

Pankreastransplantationen Ü 308

Pannikulitis Ka 340

Papillary muscle O 797

Paranitrophenol Ka 617

Paraproteins O 1183

Parasites, Immunobiology Bespr 905

Parasitic diseases O 14

Parasympathetic nervous system O 26

Parathion poisoning Ka 96

Parathion-intoxikation Ka 96

Parathormon, humanes Ka 927

Parathormonresistenz O 678

Parathyroid adenoma O 472

Parathyroid hormone O 421, O 472, O 678

Parathyroid hormone deficiency Ka 927

Paresis of the hand $\mathrm{Ka} 1195$

Paroxysmale nächtliche Hämoglobinurie Ü 247

pars plana vitrectomy O 1168

Pathologie Bespr 331

Pathology Bespr 840

Pathophysiologie Bespr 788

Pathophysiology of autoimmune diseases Suppl XXI

Patients in advanced age $\mathrm{O} 576$

$\mathrm{pCO}_{2}$ in dialysate $\mathrm{O} 806$
PDGF, platelet-derived growth factor Ü 53

-, structure of $\ddot{U} 54$

PDGF, storage of Ü 54

PDGF receptors 55

Pelvic hematoma Ka 619

Pelvic trauma, blunt $\mathrm{Ka} 619$

Penbutolol O 951

Penicillin Pharma 624

Penicillin G Ka 427, Ka 525

Penicillin V Ka 1199

Penis Pharma 581

Pentamidine O 397

Pentoxifyline O 901

Peptic ulcers O 313, O 959

Peptidhormone Ü 985

Perfusion O 224

Pericardial drainage $\mathrm{Ka} 436$

Perimyocarditis Ka 237

Peripheral ANP distribution Ü 699

Peripheral arterial disease Ka 1195

Peripheral arteriosclerosis O 518

Peripheral nerve lesion by cold EK 146

Peripheral neuropathy O 31

Peripheral vascular disease O 68, O 588, O 590, O 830

Peristaltische Aktivität O 602

Peritonitis Ka 427

Periventricular lucency O 1176

Perkutane transluminale Koronarangioplastie $\mathrm{O} 823$

Peroxidase O 33

$\mathrm{PGE}_{2}$ Kon 1138

PGH synthase Ü 54

$\mathrm{pH}$ regulation $\mathrm{O} 175$

Phäochromozytom Ü 988

Pharmaco-kinetics Pharma 623

Pharmacology in the elderly Pharma 623

Pharmacotherapy of autoimmune diseases Suppl XXI

Pharmaka, positiv inotrope Ü 887

Phenacetin-induced hemolysis O 41

Phenhydan Ka 1191

Phenhydan-Reduktion Ka 1193

Phenobarbitone Pharma 624

Phenothiazines Ü 938

Pheochromocytoma O 669

Phlebography O 1020

Phlebotomy O 18, O 24

Phosphate O 421, O 472

Phosphates Ü 359

Phosphatidylcholine Kon 1134

Phosphatidylinositol Ü 56

Phosphodiesterasehemmstoffe Ü 893

Phosphoethanolamine EK 49

Phosphoinositide Kon 1134

Phospholipase C Ü 54, Ü 56, Kon 1134

Phospholipase D Kon 1134

Phospholipids Preis-Vorlsg. 149

-, chain-length Preis-Vorlsg. 153

Phosphor O 678

Phosphorylated protein pp150 O 1003

Photon counting camera O 33

Photoplethysmographie O 1208

Phylogeny of the MHC complex Bespr 1075

Physical exercise O 951

Phytanic acid O 1183

Pimozide Ü 938

Pirenzepine O 961

Pituitary adenoma Ka 342

Pituitary insufficiency Ka 342 
Pituitary sarcoidosis Ka 342

Pituitary secretion Ü 1159

Pituitary-adrenal responses $\mathrm{O} 608$

Plasma Ü 1159

Plasma aldosterone $\mathrm{O} 75$

Plasma atrial natriuretic factor O 313

Plasma catecholamines Ka 96

Plasma cholesterol O 814

Plasma creatinine Ka 1200

Plasma exchange O 1184, O 1188

-, complications O 1183

Plasma glutathione, hepatic efflux O 1008

-, renal extraction O 1008

-, species differences O 1008

Plasma high density lipoprotein-cholesterol O 814

Plasma levels Ü 1158

Plasma lipids O 664, O 814

Plasma membrane Kon 1089

Plasma membranes of tumor cells Ü 444

Plasma norepinephrine O 269

Plasma plasmalogens EK 49

Plasma protein O 518

Plasma protein-binding Pharma 624

Plasma renin activity $\mathrm{O} 71, \mathrm{Ka} 981$

Plasma separation O 1039

Plasma triglycerides O 269, O 814

Plasma viscosity O 559

Plasma-Adrenalin Ka 99

Plasma-angiotensin II levels Kon 1127

Plasma-Katecholamine Ka 96, Ka 99

Plasmalogens in plasma EK 49

Plasmapheresis Ka 976

Plasmaspiegel O 1179

Plasmodium falciparum O 277

Plasticity EK 136

Platelet activating factor O 901

Platelet aggregation O 23, O 213

Platelet ANF receptors O 709

Platelet defects O 847

Platelet mediators, proinflammatory $\mathrm{O} 460$

Platelet-activating factor $\mathrm{O} 213$

Platelet-derived growth factor Ü 53

Platelets Ü 53, O 518, O 709, O 718, O 901, Kon 1112

-, free-calcium level 0718

Plazenta Ü 989

Pleiotropic response to Aldosterone Kon 1089

Pleural mesothelial cells Ü 53

Plexus myentericus O 602

Pneumococcal septicemia O 1187

Pneumocystis carinii Ü 300, O 372, O 397, Ka 1189

Pneumocystis carinii pneumonia O 1050

Pneumocystis-carinii-Pneumonie O 853

Pneumonia O 218, O 372, O 397

Pneumonien O 372

-, Klassifikation O 373

Pneumothorax O 1187

Poliovirus, type 1 O 914

Polyarthritis, chronische O 460

Polycythemia vera, therapy O 18

Polyglobulism O 1185

Polymerase chain reaction $\mathrm{O} 857$

Polymorphonuclear and mononuclear leukocytes Ü 54

Polymorphonuclear leukocyte Ü 53

Polyneuropathie Ka 340

Polyöstradiolphosphat Ü 351

Polypen, adenomatöse $\mathrm{O} 87$

Polypeptide O 1003

Polypeptide hormone Ü 1157
Polypharmacy Pharma 625

Polysaccharides O 896

Pontine hemorrhage O 1174

Pontine myelinolysis Ka 191

Porcine insulin O 447

Portal dopamine Ü 1157

Positiv inotrope Substanzen Ü 893

Positive inotropic agents Ü 887

Postischämische Dysfunktion O 1179

Postischämisches Myokard O 1181

Postischemic dysfunction O 1178

Postischemic hearts O 1178

Postischemic myocardium O 1178

Postmenopausal women Ü 359,0425

Postoperative thrombohaemorrhagic complications O 847

Postsecretory absorption Ka 878

Postthrombotic syndrome O 1208

Postthrombotische Syndrome O 1213

Posttransplantationslymphome Ka 1193

-, zerebrale Ka 1189

Postviral fatigue Ü 789

Potassium O 71, O 75, Ü 199, O 223

Potassium depletion O 180

Potassium intake of the elderly Pharma 625

Potassium Secretion O 233

Präeklampsie O 559

Pralidoxime Ka 615

Pränataldiagnostik Bespr 1182

Pravidel Ü 347, Ka 384

Pre-Cushing's adrenocortical adenoma O 608

Pre-damaged kidneys O 870

Prednisolon Ka 1077

Prednisolone Ka 976

Prednison Ü 250

Prednisone Ka 1071

Prednisone pulse therapy O 408

Prednisone pulses $\mathrm{O} 408$

Prednisone/Doxorubicin Ü 539

Preeclampsia O 559, O 768

Preexisting diseases 0576

Pregnancy Ü 1157, Ü 1159

Pregnantriols Ka 329

Pressure halftime method O 263

Primärtumor O 579

Primary hyperparathyroidism $\mathrm{O} 472$

Primary orthostatic hypotension EK 873

Primary sympathetic insufficiency EK 873

Procarbablastine Ü 539

Procarbazine Ü 539

Procaryotic expression O 1003

Procoagulant state $\mathrm{Ka} 281$

Progression of renal failure O 1042

Progressive systemic sclerosis O 602

Proinsulin Ü 986

Prokinetikum O 602

Prolactin Ka 342, Ü 1157, Ü 1158

Prolactin gene UU $1157, \ddot{U} 1158$

Prolactin inhibitor $\mathrm{Ka} 384$

Prolactin levels Ka 384

Prolactin secretion $\ddot{U} 1157, \ddot{U} 1162$

-, circadian rhythm Ü 1158

-, pulsatility Ü 1158

Prolactinoma Ka 384, Ü 1161

Prolactin-producing tumors Ü 1157

Prolaktinome Bespr 1049

Propafenone EK 872

Propranolol Pharma 624

Prostacyclin Bespr 48, Bespr 511 
Prostacyclin synthese Ü 54

Prostaglandin O 172, Kon 1134

Prostaglandin $E_{1} \quad O 207$

Prostaglandin $\mathrm{E}_{1}$ infusion $\mathrm{O} 380$

Prostaglandins O 172

Prostatakarzinom Ü 347

Prostatectomy Ü 347

Prostatic carcinoma Ü 347

Prosthetic mitral valve O 263

Prosthetically replaced heart valves 0921

Prostitution O 418

Protein C Ka 281

Protein C inhibitor $1 \mathrm{Ka} 281$

Protein excretion O 228, O 234

Protein kinase C Ü 54, Kon 1134

Protein PP12 Ü 989

Protein turnover O 518

Protein-anabolic effect of exercise O 380

Proteinase Kon 1145

$\alpha 1$-Proteinase inhibitor $\mathrm{O} 1054$

Proteinsynthese O 380

Proteinuria O 774

Proteinurie O 256, O 1046

Prothil Ü 347

Proximal tubular reabsorption Ka 878

Proximal tubule O 223

Prune-Belly Syndrome EK 346

Pseudo occlusion Ka 1195

Pseudocyst Ka 485

Pseudodiverticulosis Ka 187

Pseudohypoparathyroidism Ka 927

Pseudooccluded cerebral arteries Ka 1197

Psoriatic arthritis O 685

${ }^{31}$-P-Spektroskopie Ü 200

Psychiatric examination O 552

Psychiatrie, neue Arzneimittel Pharma 1085

Psychosen Pharma 1085

Psychosomatic disorders Bespr 341

Psychosomatic intervention O 77

Psychosomatische Grundversorgung Bespr 236

Psychosomatische Medizin Bespr 984

PTA Bespr 840

Puberty O 503

Pulmonary artery pressure $\mathrm{O} 823$

Pulmonary capillaries O 901

Pulmonary edema O 1187

Pulmonary embolism O 1187, O 1208

Pulmonary gasexchange $\mathrm{O} 806$

Pulmonary oxygen uptake $\mathrm{O} 806$

Puncture of artery O 1187

Purine Ü 444

Purine-rich foods Ka 874

Purulent pericarditis $\mathrm{Ka} 436$

Putaminal hemorrhage O 1174

Pygmänen, IGF-I Spiegel Ư 995

Pyridoxal-5'-phosphate O 183

Pyridoxine EK 121, EK 142, O 183

Pyridoxine hydrochloride $\mathrm{O} 183$

Pyrimethamine-sulfadoxine $\mathrm{O} 277$

Pyrimidine nucleosides Ü 444

p24-antigen $\mathrm{O} 864$

$\mathrm{P}^{32} \quad \mathrm{O} 24$

P170 U் 444

QRS complex Ü 744

Quinidine Pharma 624

Quin-2 O 718
RAA-system O 476

Radioimmunoassasy O 1013

Radiojodtherapie Pharma 640

Radiotherapy Ü 347, Ü 539

Ranitidine O 959

Rat kidney Kon 1095

Reactive hyperemia O 588

Reanimation Bespr 1182

Recanalization Ka 1195

- , spontaneous Ka 1197

$\beta_{1}$-receptor blocker O 476

Receptor-adenylate cyclase complex Ka 927

Rectal enemas O 906

Red blood cells O 18

Reduction of NSAID dosage EK 107

Reflexion rheography O 1208

Reflux esophagitis O 1059

Refluxerkrankung der Speiseröhre 0602

Regression of advanced metastatic tumors $\ddot{U} 1$

Regression of metastatic tumor Ü 5

Rejection crisis O 408

Releasing-Hormon Ka 927

Remission Ka 244

Remitted hyperthyroidism, psychopathological symptoms O 552

Remitted hyperthyroidism, neuropsychological symptoms O 552.

Renal abscess Ka 1032

Renal ammoniagenesis O 175, O 180, Kon 1096

Renal artery stenosis $\mathrm{O} 830$

Renal blood flow O 166, O 173

Renal cell cancer Ü 1

Renal cell carcinoma O 38, Kon 1102

Renal cell tumours Kon 1102

Renal collecting duct Kon 1087

Renal disease O 692

Renal failure O 38, O 269, Ka 525, O 1042

-, acute oliguric $\mathrm{Ka} 874$

Renal function EK 872

-, age-related decline Pharma 623

Renal functional reserve $\mathrm{O} 750$

Renal hemodynamics, regulation $\mathrm{O} 758$

Renal hormonal level O 172

Renal hypertension O 718

Renal inflammation $\mathrm{O} 921$

Renal insufficiency Ka 96, O 774

Renal magnesium wasting $\mathrm{O} 835$

Renal metabolism Ü 199

Renal microvessel diameters O 170

Renal microvessels O 166

Renal nerve activity O 172

Renal nitrogen metabolism Kon 1096

Renal transplantation $\mathrm{O} 38, \mathrm{O} 40, \mathrm{O} 408$

Renal tubular damage O $869, \mathrm{O} 870$

Renal tubular defects in gout $\mathrm{Ka} 874$

renale Durchblutung O 256

Renin O 75, O 583, Kon 1127

Renin activity O 313

Renin-aldosterone-system O 476

Renin-angiotensinaldosterone system O 583

Renin-Angiotensin-Aldosteron-System O 476

Renin-angiotensin-system Kon 1127

Renovascular hypertension O 830

Reocclusion EK 294

Reperfusion O 224

Reproductive system Ü 702

Resistance to chemotherapy Ü 443

Resistance to diuretics O 545 
Respiration related frequency component O 26

Resting blood flow O 589

Rethrombose O 1210

Rethromboserate O 1213

Retinal detachment Ka 1027

Retinopathie O 82

Retroperitoneal hematoma Ka 620

Rhabdomyosarkom Ü 988

Rheology Bespr 396

Rheumatic diseases, symptoms O 469

Rheumatic fever O 923

Rheumatoid arthritis Ü 492, O 685, O 774, O 923

Rheumatologie Bespr 453

Rheumatology O 466

Rhinitis $\mathrm{Ka} 881$

Rhizopus arrhizus 060

Rhizopus lipase O 60

Ribonucleic acid O 914

Right atrial pressure $\mathrm{O} 823$

RNA O 914

Salt O 655

Salvage-treatment Ü 539

Salzsensitivität O 661

Sammelrohrzellen, papilläre Ü 200

Sarafotoxin O 758

Sarcoidosis Ka 335, Ka 342, O 1217

Sarkoidose Ka 340

-, atypische Manifestation Ka 335

Sarkoidose mit Myositis Ka 340

Säure-Hämolyse-Test Ü 249

Saving of NSAID EK 107, EK 116

Sceletal muscle O 380

Schaltstück Kon 1110

Schilddrüsenautonomie Pharma 640

-, operative Therapie Pharma 641

Schilddrüsenfunktionsstörung Pharma 635, O 964

Schizophrenie Pharma 1085

Schmerztherapie EK 103

Schwangerschaft O 768

Schwangerschaftshypertonie O 559, O 768

Scintiscan O 472

Secondary hypertension Kon 1119

Second-degree AV block O 1187

Self-rating questionnaires O 554

Sensomotor polyneuropathy $\mathrm{Ka} 50$

Sepsis O 38, Bespr 525, Bespr 1202

Septic shock Ü 841

Septicemia Ka 427

Septicemia/opportunistic infection O 1185

Serine proteinases Kon 1145, Kon 1147

Serine/threonine kinase Ü 54

Serotonin Ka 244, O 461

Serotonin receptor binding EK 142

Serotoningehalt der Thrombozyten $\mathrm{O} 463$

Serous cystadenoma Ka 780

Serum Ka 324

Serum albumin Pharma 624

Serum calcium O 678

Serum cortisol $\mathrm{Ka} 342$

Serum creatinine O 870, O 1042, Ka 1198, Ka 1200

Serum creatinine levels $\mathrm{O} 421$

Serum enzymes O 565

Serum immune complexes, test systems Ü 302

Serum levels Ü 1157

Serum lipids 065

Serum lipoproteins O 269
Serum osmolality O 559

Serum-IGF Spiegel Ư 988

Serum-Immunglobuline O 89

Serum-Kreatinin O 256

Serumprolaktinspiegel Ka 384

Sex with HIV seropositive partners O 418

Sexual activity in the USA O 908

Sexualhormone Ü 987

Sexually transmitted diseases EK 1082

Shaldon catheter O 1187

Sharp's syndrome O 14

Shock Ka 427, Ka 525, Bespr 1202

Shortened bowel O 678

Shulman-Syndrom Ka 340

Silent adrenal tumors (incidentaloma) $\mathrm{Ka} 328$

Simvastatin O 814

SIN 1 O 213

Sinusitis Ka 881

Skeletal myoblasts Ü 53

Skelettmuskel O 380

Skelettmuskulatur Ka 340

Skin Ü 489

Skin microcirculation O 207

Skin surface oxygen pressure fields O 207

Skin-tags 083

Sklerodermie, progressive systemische $\mathrm{O} 602$

Skrotalhaut Pharma 581

Sleep O 320

Small cell lung cancer O 728

Small intestine Ka 290

Small intestine surgery Ka 290

Smokers O 512

Smoking O 41

Smoking habits O 857

Smooth muscle cells Ü 53

Sodium O 71, O 75, Ü 199, O 655

Sodium chloride O 664

Sodium nitroprusside O 215

Sodium reabsorption, distal O 226

Sodium restriction $\mathrm{O} 664$

Sodium substitution Ka 191

Sodium Transport O 225

Sokolow-index O 256

Somatomedins Ü 985

Somatotropic hormone O 951

Sorbitol Ü 199, Kon 1091

Sorbitstoffwechsel bei Diabetes mellitus Ü 203

Spasm of the pelvic vessels $\mathrm{Ka} 621$

Spasmodic torticollis Ü 938

Spätmyopathie Ka 340

Spätpotentiale Ü 744

Spectral analysis O 26, Ka 1197

Spectral analysis of heart rate variability O 26

Spectrotemporal mapping Ü 744

Spektralanalyse des EKG Ü 744

Spinal dorsal horn EK 125

Spironolacton Ü 354

Spironolactone O 545

Spleen O 847

Splenectomy Ka 95, O 847

Splenomegaly O 847, O 921

Spondylarthropathy Kon 1150

St. Jude prostheses O 263

S-T wave Ü 744

Staging systems O 89

Stammganglien, Verkalkungen Ka 928

Staphylococcus aureus Ka 436, O 921 
Stereotaxy Ü 935

Steroidhormonbiosynthese O 597

Steroids Ka 94

Stimulatory guanine nucleotide-binding protein Ü 887

Stoffwechselkontrolle O 77

Stoffwechselstörungen Ü 998

Streptococcal antigens $\mathrm{Ka} 1200$

Streptococcal infection Ka 1198

Streptococcal pyrogenic toxins Ka 427

Streptococcus Ka 523, O 921

Streptococcus pyogenes Ka 427

Streptozotocin-induced defect in tubular urate reabsorption $\mathrm{Ka} 878$

Stress Ü 1157, Ü 1160

Struma Bespr 886

24-Stunden-Blutdruckmessung Kon 1119

Subarachnoid hemorrhage O 1174

Subclinical hyperthyroidism O 964

Subclinical hypothyroidism O 964

Sucrose-Test Ü 250

Sulfation factor Ü 985

Surface antigens 0728

Surgery, via the transnasal approach Ka 343

Suxamethoniumchloride Ka 615

Sympathetic activity O 269

Sympathetic blockade O 269

Sympathetic nervous system O 26, Ư 887

Sympathetic neurone blockade O 269

Symptomatic peripheral vascular disease of the lower extremity O 41

Synovial fluid $\mathrm{O} 685$

Synovial tissue Kon 1150

Syphilis O 906, EK 1082

Systemic lupus erythematodes O 14, Ü 493

Systemic lupus erythematosus O 689, O 1066

Systemic polymorphous immunocytoma Ka 1027

Systemischer Lupus Erythematodes, Autoantikörper gegen die Komplementkomponente C1q O 1066

T cell ontogeny $\ddot{U} 489$

T cell receptor $\gamma \delta$ Ü 489

$\mathrm{T}$ helper cells $\mathrm{O} 408$

T lymphocytes O 393, O 408

- , activated O 393

-, cytotoxic O 393

T suppressor-inducer cells $\mathrm{O} 408$

Tachycardia EK 441, O 957, O 1171, O 1186

Tagamet Ü 347

Target organ damage in essential hypertension O 256

TAT Ka 281

Taurocholate O 60

Taurodeoxycholate $\mathrm{O} 60$

T-cell, growth factor $\ddot{U} 3$

T-cell activation Bespr 440, O 864

T-cell receptor Ü 1

T-cell subsets $\mathrm{O} 43$

Tc-99m/T1-201 subtraction O 472

Tegretal Ka 1191

Telangiectasia, hereditary hemorrhagic Ka 290

Tergurid Ka 384

Terguride $\mathrm{Ka} 384$

Testaussagen Ed 297

Testosterone Ü 347

Tetracycline Pharma 624

Tetraiodothyronine Ka 342

Thalamic hemorrhage $\mathrm{O} 1174$

Thalamus EK 129
T-Heferzellzahl O 89

Thermodilution O 263, O 264

Thiamine EK 121

Thiopental Ka 286

Thirst regulation Kon 1127

Thrombektomie, venöse O 1213

Thrombin O 460

Thrombin-antithrombin-III-complexes Ka 281

Thrombocyte levels O 594

Thrombocytopenia Ü 247, O 1050

Thrombocytosis O 23

Thromboseneigung Ü 247

Thrombosis Ü 247, O 594, O 1186

-, catheter-related venous O 1187

Thrombospondin mRNA Ü 54

Thromboxanes O 172

Thrombozyten O 521

Thrombozytenfunktion $\mathrm{O} 460$

Thrombozytensubstitution $\mathrm{Ka} 333$

Thrombozytopenie O 89

Thrombuswachstum O 1213

Thymus Ü 489

Thyreotoxische Krise Pharma 650

Thyreotropin releasing hormone Ü 1157, Ü 1160

Thyreotropin-Releasing-Hormon Ka 927

Thyroglobulin antibodies O 1037

Thyroid C-Cells Ü 359

Thyroid crisis O 1037

Thyroid disorders O 964

-, emotional disturbances O 964

Thyroid hormone O 942

Thyroid hormones and central nervous structures O 557

Thyroid stimulating hormone Ka 342, O 964, O 1037

Thyroid storm with neurological symptoms O 1037

Thyroid volume $\mathrm{O} 503$

Thyrotoxic crisis in Graves' disease, surgery O 1037

Thyrotoxicosis O 1037

Thyrotropin-releasing hormone O 964, O 1013, O 1037

Thyroxinebinding globulin O 1015, O 1037

TIMP Kon 1145

Tissue oxygen tension $\mathrm{O} 18$

Tissue sections $\mathrm{O} 33$

T-lymphocyte O 728

TNM-Klassifikation maligner Tumoren Bespr 984

Tobramycin Ka 525

Tonic spasms Ü 935

Tonsillenvergrößerung Ka 1190

Toxic shock syndrome $\mathrm{Ka} 427$

Toxic shock-like syndrome $\mathrm{Ka} 427$, Ka 523

Toxoplasmen Ka 1189

Trace elements O 507

Transepithelial ion transport Kon 1087

Transferrin $\mathrm{O} 728$

Transformed cells Ü 53

Transfusion U் 250, O 1050, EK 1082

Transfusionsmedizin Bespr 1156

Transplantation of pancreatic tissue Ü 306

Transprosthetic flow calculation O 267

Trauma Bespr 1202

Travellers O 277

TRH/Dopamine agonist drugs Ü 1157

Triacylglycerol hydrolase O 60

Tricyclic antidepressants Pharma 624

Triglyceride O 664

Triglyceride-levels $\mathrm{O} 83$

Triglycerides O 60, O 65

Triglyceridkonzentration O 85, O 86 
Triglyceridlipolyse O 380

Triiodothyronine Ka 342

TRIS Ka 286

Tropical malabsorption Ü 493

Trypsin O 565

TSH secretion O 1013

TSH-receptor antibodies O 1037

Tuberculosis Ka 344

Tubular destruction O 166

Tubular lesions, postischemic diagnostic localization O 223

Tubular osmolyte synthesis Kon 1091

Tubular secretion further distally $\mathrm{Ka} 878$

Tubulointerstitial nephritis $\mathrm{Ka} 971$

Tubulovilous adenoma $\mathrm{O} 83$

Tumor cell resistance $\ddot{U} 443$

Tumor cells O 896

Tumor infiltrating lymphocytes Ü 1

Tumor marker Ka 780

Tumor necrosis factor Ü 3, Ka 281, Ü 300, Ü 841, O 901

Tumor necrosis factor $\alpha$ Ü 1 , EK 1083

Tumor of the heart O 1059

Tumor of the kidney $\mathrm{Ka} 1032$

Tumor progression $\mathrm{Ka} 241$

Tumor tissues O 33

Tumoren, IGF-II Spiegel Ü 996

Tumor-infiltrating lymphocyte $\ddot{U} 1$

Tumor-infiltrating lymphocytes Ü 1

Tumorlokalisationsschlüssel Bespr 190

Tumor-necrosis factor $\beta$ Ü 1

Tumornekrosefaktor TNF- $\alpha$ Ka 281

Type I astrocytes Ü 53

Type I diabetes O 447, O 750

Type III HLP O 68

Type III hpyerlipoproteinemia O 65, Ka 526

Type 1 hypersensitivity to Azathioprine Ka 50

Tyrosine kinase $\ddot{U} 54$

Tyrosine kinase activation Ü 56

T4 lymphocytes O 397

T4/T8 ratio $\mathrm{O} 728$

T4/T8-Ratio O 89

T1-2012 SPECT O 472

Ulcera der Extremitäten $\mathrm{O} 383$

Ulcers O 207

Ulcus cruris O 1211

Ulkuskrankheit EK 488

Ultraschall, Innere Medizin Bespr 840

-, interventioneller Bespr 25

Ultraschalldiagnostik, abdominale Bespr 773

Ultraschall-Dopplersonographie O 1208

Ultraschalltomographie des Abdomens Bespr 402

Ultrasonographic volumetry O 503

Unresponsive to corticosteroid treatment $\mathrm{Ka} 1030$

Uptake of $\mathrm{CO}_{2}$ from the dialysate $\mathrm{O} 806$

Urea cycle capacity $\mathrm{O} 175$

Urea synthesis O 175, Kon 1096

Uremia O 183

Uremia Therapy Bespr 757

Ureogenesis O 175

Uric acid O 559, O 1222

Uric acid crystals Ka 874 , Ka 877

Urinary ammonium excretion O 180

Urinary catecholamines Ka 96

Urinary iodine $\mathrm{O} 503$

Urinary potassium excretion $\mathrm{O} 228$

Urinary protein excretion O 256

Urinary undissociated uric acid Ka 874
Urine Ka 324

Urine alkalinization Ka 879

Urinelektrophorese $\mathrm{Ka} 329$

Urin-Katecholamine Ka 96

Urinvolumen $\mathrm{O} 661$

Urologie Bespr 435

Uveitis Ka 1027

-, chronic Ka 1027

Vagina Pharma 581

Validierung diagnostischer Tests Ed 297

Valvular disease O 1174

VAL-1 O 728

Varicella-zoster virus Ka $1198, \mathrm{Ka} 1200$

Vascular complications EK 49

Vascular dementia O 1176

Vascular mechanisms O 166

Vascular resistance O 170

Vascular smooth muscle cells O 801, Kon 1112

Vasculitis O 1040

Vasculitis syndrome $\ddot{U} 300$

Vasoactive drugs O 1168

Vasoactive intestinal peptide Ü 1157

Vasoconstriction Ü 54, O 166, O 170, O 801

Vasoconstrictor Ü 53, O 758

Vasokonstringierender Effekt O 1180

Vasopressin Kon 1127

Vasopressin treatment Kon 1091

Venenklappen O 1213

Venerische Infektionen Bespr 487

Venogram Ka 620

Venous $\mathrm{PO}_{2}$ levels $\mathrm{O} 210$

Venous thrombectomy O 1208

Venous thrombosis O 1185

Ventricular dysfunction, left $\mathrm{O} 1178$

Ventricular fibrillation $\mathrm{O} 1171$

Ventricular tachycardia $\ddot{U} 744$

Ventriculoatrial shunt $\mathrm{Ka} 485$

Ventriculoperitoneal shunt $\mathrm{Ka} 485$

Verapamil Ü 444, O 797

Very low density lipoprotein-cholesterol O 814

Very low-density lipoproteins $\mathrm{O} 65$

Vesicles Ka 1200

Vinblastine Ü 539

Vincristin Ka 1077

Vincristine Ü 539

Viral infection O 218

Viral infections, somatopsychic symptoms Ü 789

Viral infections of the CNS O 12

Viscotoxins O 896

Viscum album O 896

Vitamin B EK 136

Vitamin B combination EK 129

Vitamin B complex EK 121

Vitamin $B_{1}, B_{6}, B_{12}$ EK 103, EK 107, EK 116

Vitamin $\mathrm{B}_{1}, \mathrm{~B}_{6}, \mathrm{~B}_{12}$-treatment EK 146

Vitamin $\mathrm{B}_{6}$ EK 129, O 183

Vitamin B6 deficiency in uremia O 185

Vitamin $B_{12}$ EK 121

Vitamin D O 421, Ka 929

Vitamin D-Resistenz O 678

Vitamine Bespr 471

Vitamin-K dependent coagulation factors O 1186

VLDL O 65

VLDL remnants 067

VLDL-cholesterol O 65

VLDL-cholesterol/serum-triglyceride ratio Ka 526 
Volume regulation Ü 199

Volume retaining disorders 0709

Vomiting Ka 50 , Ka 525

von Willebrand's disease O 1203

Vulva Pharma 581

Vulvabehandlung mit lokalen Östrogenen Pharma 581

Wachstum Ü 985, Ü 992, Ü 998

Wachstumsfaktoren $\ddot{U} 985$

-, Wirkmechanismen Ư 989

Wachstumshormon Ü 987

Wachstumsschub Ka 330

Waking up O 320

Walter Reed, Stadieneinteilung O 865

Walter-Reed-classification O 89, O 393

Water and salt homeostasis Ü 699

Wegener's granulomatosis $\mathrm{Ka} 880$

Western blot O 899, O 907

Wilms-Tumor Ü 988

Wismut-Absorption EK 488

Wismut-Konzentrationen EK 488

Wismut-Präparate, Pharmakokinetik EK 488
Wismut-Subsalizylat Suspension EK 488

Wismut-Subzitrat Filmtabletten EK 488

Wound healing $\ddot{U} 53$

WR1 O 393

WR2 O 393

Wundheilung O 383, Ü 998

Xanthomatosis Ka 526

Xenobiotics U் 443, Ü 444

Xenopus embryo Ü 53

Xipamide O 545

-, side effects O 545

Yersinia arthritis 0685

Zellproliferation Ü 991

Zerebrale Schädigung durch Hyponatriämie Ka 192

Zigarettenrauchen Bespr 430

Zinc $\mathrm{O} 507$

Zirrhose Ka 1034

ZNS-Komplikationen Ka 1190

Zytomegalie-Virus, humanes Ka 1189 



\section{Autorenregister}

Die halbfetten Zahlen bezeichnen Originalarbeiten, die Zahlen in gewöhnlicher Schrift Buchbesprechungen

Aigner $\mathrm{F}, \rightarrow$ Sandbichler $\mathrm{P}$, et al. 290

Albus $\mathrm{C}$, Ollenschläger $\mathrm{G}$, Thomas W, Fischer H, Schäfer HM, Peters R $\mathbf{7 7}$

Alexander $\mathrm{K}, \rightarrow$ Creutzig A, et al. 207

Allolio B, $\rightarrow$ Fätkenheuer G, et al. 89

Altwein JE, Faul P 347

Amelung $\mathrm{F}, \rightarrow$ Schröder JM, et al. 345

Angermann $\mathrm{CE}, \rightarrow$ Spes $\mathrm{CH}$, et al. 872

Ansorge G, $\rightarrow$ Eisenhauer T, et al. $\mathbf{7 5 0}$

Antes U, Heinz HP, Hartung $\mathrm{K}$, Loos M 1066

Antoku Y, $\rightarrow$ Goto I, et al. 49

Antoni $\mathrm{H}, \rightarrow$ Lüderitz B 517

Arnold $\mathrm{H}, \rightarrow$ Mißler U, et al. 342

Arntz H-R, $\rightarrow$ Sharma AM, et al. 664

Atlan $\mathrm{H}, \rightarrow$ Sigal $\mathrm{R}$, et al. 396

Atzpodien J, Kirchner $\mathrm{H} \quad 1$

Aulitzky WE, $\rightarrow$ Tilg $\mathrm{H}$, et al. 1083

Autenrieth G, $\rightarrow$ Scheidt W von, et al. 241

Avenarius $\mathrm{HJ}, \rightarrow$ Horn $\mathrm{A}$, et al. 460

Bachmann M, $\rightarrow$ Mayet W-J, et al. 685

Bäcker $\mathrm{A}, \rightarrow$ Kramer $\mathrm{HJ}$, et al. 1112

Badoz A, $\rightarrow$ Wackenheim A 773

Bahner $\mathrm{U}, \rightarrow$ Heidbreder E, et al. 1127

Bähr V, $\rightarrow$ Hensen $J$, et al. 608

Ballé C, Schollmeyer P 38

Barleben $\mathrm{H}$, Wagenknecht $\mathrm{C}$, Jung $\mathrm{K}$, Junker L, Reimann $\mathrm{H}$, Hamann $\mathrm{K}$, Heine H 518

Barth CA, Fürst P 392

Bartoszyk GD 121

Bässler KH 471

Baum P, $\rightarrow$ Schuster HP, et al. 1182

Baumeister $\mathrm{H}, \rightarrow$ Eiff $\mathrm{M}$ von, et al. 372

Baumgart P, Rahn KH 320

Baumgart $P$, Walger $P$, Jürgens U, Rahn KH $\mathbf{7 2 3}$
Baumgart $\mathrm{P}, \rightarrow$ Eiff $\mathrm{M}$ von, et al. 372

Baumgartner FJ, Goodnight J, Nordestgaard AG, White G 619

Baumgartner FJ, Moore TC, Mitchner J 485

Baur X, $\rightarrow$ Hauck R 512

Bäcker $U, \rightarrow$ Bogner JR, et al. 1050

Beahrs OH, $\rightarrow$ Spiessl B, et al. 984

Beck A, $\rightarrow$ Rosen A, et al. 537

Beck FX, $\rightarrow$ Guder WG, et al. 1091

Becker $\mathrm{KW}, \rightarrow$ Kienecker EW, et al. 146

Becker W 640

Benker G, Breuer N, Wehr M 631

Benker G, Jaspers C, Häusler G, Reinwein D 1157

Benker $G, \rightarrow$ Kraatz C, et al. $\mathbf{5 8 3}$

Beretta-Piccoli C, $\rightarrow$ Weidmann $P$, et al. 269

Berg PA, $\rightarrow$ Stettin A, et al. 896

Bergmann G 236

Bergner $D, \rightarrow$ Harrer $T$, et al. 864

Bernardi $\mathrm{P}, \rightarrow$ Fontana $\mathrm{F}$, et al. 313

Beyer J-H, $\rightarrow$ Kaboth U, et al. 18

Beyer $\mathbf{J}, \rightarrow$ Weber $\mathrm{T}$, et al. 951

Beyer M, $\rightarrow$ Hoffmeister HM, et al. 1178

Bienzle $\mathrm{U}, \rightarrow$ Deininger $\mathrm{S}$, et al. 906

Bienzle U, $\rightarrow$ Kiese M, et al. 1082

Bienzle U, $\rightarrow$ Stark K, et al. 415

Bigge J, $\rightarrow$ Kaboth U, et al. 18

Bilger R 773

Binder $\mathrm{T}, \rightarrow$ Wieshammer $\mathrm{S}$, et al. 436

Binswanger U, $\rightarrow$ Hess B 874

Binswanger $U, \rightarrow$ Kiparski A v, et al. 408

Bircher J, Lotterer E 475

Biro $G$, $\rightarrow$ Leicht $E$, et al. 678

Blaas $P$, Weber $S$, Hänsch GM, Peter HH 247
Blessing $\mathrm{J}, \rightarrow$ Raedsch $\mathrm{R}$, et al. $\mathbf{4 8 8}$

Bode JC, $\rightarrow$ Walker S, et al. 959

Bode $\mathrm{M}, \rightarrow$ Wieshammer $\mathrm{S}$, et al. 436

Bogner JR, Gathof B, Heinrich $B$, Matuschke A, Bäkker U, Goebel F-D 1050

Bogner JR, Matuschke A, Heinrich B, Schreiber MA, Nerl C, Goebel F-D 393

Bogner JR, Zoller WG, Middeke M 1032

Bogner JR, $\rightarrow$ Zoller WG, et al. 830

Bogunovic N, Mannebach $\mathrm{H}$, Ohlmeier H 532

Böhm M, Schwinger RHG, Erdmann E $\mathbf{8 8 7}$

Böhm $\mathrm{M}, \rightarrow$ Scheidt $\mathrm{W}$ von, et al. 241

Böhm M, $\rightarrow$ Schwinger RHG, et al. 797

Bommer J, $\rightarrow$ Feussner G, et al. 65

Bommer $M$, Eversmann $T$, Pickardt R, Leonhardt A, Naber D $\mathbf{5 5 2}$

Bonke D, $\rightarrow$ Dakshinamurti $\mathrm{K}$, et al. 142

Bonke D, $\rightarrow$ Dimpfel W, et al. 136

Bonke D, $\rightarrow$ Jurna I, et al. 129

Bönner G, Rahn KH 48, 511

Borgis $\mathrm{K}-\mathrm{J}, \rightarrow$ Mißler $\mathrm{U}$, et al. 342

Borisch B, $\rightarrow$ Jahn G, et al. 1003

Born GVR, Cuatrecasas P, Herken H, Schwartz A 182

Borries M, $\rightarrow$ Schrader J, et al. 774

Brackmann $\mathrm{HH}, \rightarrow$ Kamradt T, et al. 1203

Brandstetter BM, $\rightarrow$ Wenger R 190

Braun $\mathrm{J}, \rightarrow$ Wees $\mathrm{J}$ van, et al. 1054

Bretschneider HJ, $\rightarrow$ Kehrer G 223

Breuer $N, \rightarrow$ Benker G, et al. 631

Brockmeyer NH, Mertins L, Goos M 1229

Bröker B, Emmrich F 489

Bröker M, $\rightarrow$ Jahn G, et al. 1003
Brüggemann $\mathrm{G}$, Koehler $\mathrm{CO}$, Koch EMW 116

Brune GG, $\rightarrow$ Hofferberth B 580

Brune S, $\rightarrow$ Schmidt T, et al. 294

Brussee H, $\rightarrow$ Eber B, et al. 594

Buchkremer G, $\rightarrow$ Tölle R 430

Büchner $\mathrm{C}, \rightarrow$ Lehmann $\mathrm{M}$, et al. 873

Buhl $\mathrm{M}, \rightarrow$ Hensen $\mathrm{J}$, et al. 608

Buhr-Schinner $\mathrm{H}, \rightarrow$ Rath W, et al. 768

Bunjes D, $\rightarrow$ Schmidt A, et al. 286

Buscher H-P 443

Buschmann-Kaspari $\mathrm{H}, \rightarrow$ Lankisch PG, et al. 565

Caird FI 623

Campbell WC 926

Cantero Hinojosa $\mathrm{J}, \rightarrow$ Garcia Ontiveros A, et al. 496

Cantero Hinojosa J, $\rightarrow$ Ruiz Martínez M, et al. $\mathbf{5 0 7}$

Capelli $\mathrm{M}, \rightarrow$ Fontana $\mathrm{F}$, et al. 313

Carlsson K-H, $\rightarrow$ Jurna I, et al. 129

Caspary L, $\rightarrow$ Creutzig A, et al. 207

Caspary WF, $\rightarrow$ Wehrmann T 602

Castillo-Höfer C, $\rightarrow$ Lorenz J, et al. 728

Castro $\mathrm{S}$ de, $\rightarrow$ Laso Guzmán FJ, et al. 183

Cava F, $\rightarrow$ Laso Guzmán FJ, et al. 183

Chapman $\mathrm{N}, \rightarrow$ Wiegand V, et al. 914

Christen RD, Moser R, Schlup R, Neftel KA $\mathbf{4 2 7}$

Clarmann M v, $\rightarrow$ Kauert B, et al. 96

Classen $\mathrm{M}, \rightarrow$ Lersch $\mathrm{C}$, et al. 523

Colardyn F, $\rightarrow$ Wilde V de, et al. 615

Coles GA, $\rightarrow$ Davies $M$, et al. 1145

Comella CL, $\rightarrow$ Diederich N, et al. 935

Cordes $U, \rightarrow$ Weber T, et al. 951

Cotton PB, Williams C 926 
Cremer H-D, Heilmeyer L, Holtmeier H-J, Hötzel D, Kühn HA, Künau J, Zöllner N 523

Creutzfeldt $\mathrm{C}, \rightarrow$ Thiery $\mathrm{J}$, et al. 814

Creutzfeldt W, $\rightarrow$ Löser Chr, et al. $\mathbf{7 8 0}$

Creutzfeldt W, $\rightarrow$ Thiery J, et al. 814

Creutzig A, Caspary L, Alexander K 207

Cruse JM, Lewis RE Jr 402

Cuatrecasas P, $\rightarrow$ Born GVR, et al. 182

Daerr W, $\rightarrow$ Grosser S, et al. 806

Dakshinamurti K, Sharma SK, Bonke D 142

Damjanov I, $\rightarrow$ Rubin E 840

Davies M, Coles GA, Thomas GJ, Martin J, Lovett DH 1145

Degenhardt S, $\rightarrow$ Fätkenheuer $G$, et al. 89

Deicher $\mathrm{H}, \rightarrow$ Horn A, et al. 460

Deininger S, Müller R, Guggenmoos-Holzmann I, Laukamm-Josten U, Bienzle U 906

Deininger $S, \rightarrow$ Stark K, et al. 415

Dengler HJ, $\rightarrow$ Ewig S 789

Denz H, Fuchs D, Hausen A, Huber $H$, Nachbaur $D$, Reibnegger G, Thaler J, Werner ER, Wacher $\mathrm{H}$ 218

Deutsch E, Dienstl F, Kleinberger $G$, Laggner AN, Lenz K, Ritz R, Schuster HP 788

Dick P, $\rightarrow$ Kienecker EW, et al. 146

Diederich $\mathrm{C}$, $\rightarrow$ Weber $\mathrm{T}$, et al. 951

Diederich N, Goetz CG, Comella CL 935

Diehl V, Pfreundschuh $\mathrm{M}$, Loeffler M 905

Dienstl F, $\rightarrow$ Deutsch E, et al. 788

Dienstl F, $\rightarrow$ Tilg $\mathrm{H}$, et al. 1083

Dierich MP, $\rightarrow$ Reibnegger $\mathrm{G}$, et al. 43

Dietrich M 276

Dietrich $\mathrm{M}, \rightarrow$ Hemmer ChJ, et al. 281

Dimpfel W, Spüler M, Bonke D 136

Distler A, $\rightarrow$ Sharma AM, et al. 664

Dittmann $H$, Voelker W, Karsch K-R, Seipel L 263
Dobos GJ, Meske S, Keller E, Riegel W, Vaith P, Peter HH, Schollmeyer P 976

Doboszýnska A, $\rightarrow$ Lorenz J, et al. 728

Doehn $\mathrm{M}, \rightarrow$ Knüttgen $\mathrm{D}$, et al. 1168

Doering $\mathrm{W}, \rightarrow$ Huhn $\mathrm{C}$, et al. 853

Domellöf L 536

Dörfler $H$, Permanetter $W$, Küffer $G$, Häußinger $K$, Zöllner N 388

Doyon D, $\rightarrow$ Sigal $R$, et al. 396

Drews J 1207

Duntas L, Keck FS, Rosenthal $\mathrm{J}$, Wolf $\mathrm{Ch}$, Loos $\mathrm{U}$, Pfeiffer EF 1013

Dusleag J, $\rightarrow$ Eber B, et al. 594

Eber B, Kronberger-Schaffer E, Brussee H, Klima G, Obernosterer A, Toporsch M, Dusleag J, Klein W 594

Eber B, $\rightarrow$ Lind $\mathrm{P}$, et al. $\mathbf{4 7 2}$

Eber $\mathrm{O}, \rightarrow$ Lind $\mathrm{P}$, et al. $\mathbf{4 7 2}$

Eckert B, $\rightarrow$ Schaaf L, et al. 669

Eckhardt A 198

Egbring RE, $\rightarrow$ Hemmer ChJ, et al. 281

Eggeling $\mathrm{T}, \rightarrow$ Schmidt A, et al. 286

Eggstein M, $\rightarrow$ Jakober B, et al. 447

Eichenlaub D, $\rightarrow$ Huhn C, et al. 853

Eiff $M$ von, Steimann $R$, Roos N, Husen N van, Walger P, Baumgart P, Fegeler $\mathrm{W}$, Junge $\mathrm{E}$, Baumeister $\mathrm{H}$, Wilms $\mathrm{B}$, Heinicke A, Loo J van de 372

Eisenhauer T, Jungmann E, Warneboldt D, Ansorge G, Scherberich J, Talartschik J 750

Emmrich F, $\rightarrow$ Bröker B 489

Endres W, $\rightarrow$ Schmittenbecher PP 346

Erdman E, $\rightarrow$ Schwinger RHG, et al. 797

Erdmann E, $\rightarrow$ Böhm M, et al. 887

Eversmann T, $\rightarrow$ Bommer M, et al. 552

Ewig S, Dengler HJ 789

Eyrich K, $\rightarrow$ Reinhart K 525

Faber J-P, $\rightarrow$ Poller W, et al. 857

Faist E, Ninnemann JL, Green DR 1202
Fandrey J, $\rightarrow$ Rob PM 835

Färber $P, \rightarrow$ Wood WG, et al. 324

Fätkenheuer G, SchrappeBächer M, Salzberger B, Degenhardt S, Allolio B 89

Faul $\mathrm{P}, \rightarrow$ Altwein JE 347

Feddersen $\mathrm{CO}, \rightarrow$ Schmidt JA, et al. 191

Fegeler W, $\rightarrow$ Eiff $M$ von, et al. 372

Fehr K, Miehle W, Schattenkirchner $\mathrm{M}$, Tillmann $\mathrm{K}$ 453

Felgenhauer $\mathrm{K}, \rightarrow$ Rieckmann $\mathrm{P}$, et al. 12

Felgenhauer $\mathrm{N}, \rightarrow$ Kauert $\mathrm{B}$, et al. 96

Felix R, $\rightarrow$ Lüning M 1075

Ferlinz R, $\rightarrow$ Lorenz J, et al. 728

Ferrari $\mathrm{P}, \rightarrow$ Weidmann $\mathrm{P}$, et al. 269

Feussner G, Bommer J, Ziegler $\mathrm{R} 65$

Feussner G, Hodenberg E von, Ziegler R 526

Fierz W, $\rightarrow$ Kiparski A v, et al. 408

Fischer $\mathrm{H}, \rightarrow$ Albus $\mathrm{C}$, et al. 77

Flückiger R 102

Földes $\mathrm{O}, \rightarrow$ Tajtáková $\mathrm{M}$, et al. 503

Fölsch UR, $\rightarrow$ Löser $\mathrm{Chr}$, et al. $\mathbf{7 8 0}$

Fontana F, Bernardi P, Spagnolo $N$, Capelli $M \quad 313$

Forth W, $\rightarrow$ Zöllner N 103 , 105

Franke-Raue $\mathrm{K}$, Junga $\mathrm{G}$, Raue F, Vecsei P, Ziegler R 597

Frei D, $\rightarrow$ Kiparski A v, et al. 408

Frei G, $\rightarrow$ Kiparski A v, et al. 408

Fretschner $\mathrm{M}, \rightarrow$ Zimmerhackl LB, et al. 166

Fric M, Hartmann A, Klehr H-U, Pfeifer U, Herberhold C 1189

Friedrich J, $\rightarrow$ Schmidt A, et al. 286

Frommhold W, $\rightarrow$ Gerhardt $P 435$

Fu Q-G, Sandkühler J, Zimmermann M 125

Fuchs D, $\rightarrow$ Denz $\mathrm{H}$, et al. 218

Fuchs D, $\rightarrow$ Reibnegger G, et al. 43

Fuchs E, $\rightarrow$ Gröne H-J, et al. 758

Fuhrer JA, Mihatsch MJ, Streuli RA 1198
Fujishima $\mathrm{M}, \rightarrow$ Yoshinari $M$, et al. 441

Fürst P, $\rightarrow$ Barth CA 392

Gain T, $\rightarrow$ Lersch C, et al. 523

Garcia Ontiveros A, Cantero Hinojosa J, Gil Extremera $\mathrm{B}$, Miñarro del Moral J 496

Gathof B, $\rightarrow$ Bogner JR, et al. 1050

Gathof B, $\rightarrow$ Gresser U, et al. 1222

Gattermann N, $\rightarrow$ Thomas M, et al. 539

Gehrig W, $\rightarrow$ Weise F, et al. 26

Geiger $\mathrm{H}, \rightarrow$ Heidbreder E, et al. 1127

Geiling $\mathrm{H}, \rightarrow$ Weber $\mathrm{T}$, et al. 951

Geissler W, $\rightarrow$ Schaaf L, et al. 669

Gerber H, $\rightarrow$ Müller P, et al. 332

Gerhardt P, Frommhold W 435

Gerok W, $\rightarrow$ Häussinger D, et al. 175

Gerok W, $\rightarrow$ Knauf $\mathrm{H}$, et al. 545

Gerzer R, $\rightarrow$ Heim J-M, et al. 709

Gerzer R, $\rightarrow$ Karrenbrock B, et al. 213

Giani G, $\rightarrow$ Kindler J, et al. 1042

Gil Extremera B, $\rightarrow$ Garcia Ontiveros A, et al. 496

Gil Extremera B, $\rightarrow$ Ruiz Martínez M, et al. $\mathbf{5 0 7}$

Glasmacher AG, $\rightarrow$ RobertzVaupel GM, et al. 1076

Glück $\mathrm{H}, \rightarrow$ Jakober $\mathrm{B}$, et al. 447

Glück S, $\rightarrow$ Söhngen $D$, et al. 1071

Goebel F-D, $\rightarrow$ Bogner JR, et al. 393, 1050

Goedert JJ, $\rightarrow$ Reibnegger $\mathrm{G}$, et al. 43

Goerg KJ, $\rightarrow$ Knauf $\mathrm{H}$, et al. 545

Goës R, $\rightarrow$ Walker S, et al. 187

Goetz CG, $\rightarrow$ Diederich N et al. 935

Gonzalez-Buitrago JM, $\rightarrow$ Laso Guzmán FJ, et al. 183

Goodnight J, $\rightarrow$ Baumgartner FJ, et al. 619

Goos $\mathrm{M}, \rightarrow$ Brockmeyer NH, et al. 1229

Goronzy JJ, Weyand CM 735 
Goto I, Antoku Y, Hayakawa T 49

Gottmann K, $\rightarrow$ Heim J-M, et al. 709

Götz R, $\rightarrow$ Heidbreder E, et al. 1127

Grabensee B, $\rightarrow$ Plum J, et al. 476

Green DR, $\rightarrow$ Faist E, et al. 1202

Greschner M, $\rightarrow$ Schaaf L et al. 669, 1037

Gresser U, Gathof B, Zöllner N 1222

Gresser U, $\rightarrow$ Zöllner N, et al. 101

Greten $\mathrm{H}, \rightarrow$ Grosser $\mathrm{S}$, et al. 806

Gröne H-J, Laue A, Fuchs E 758

Gröne H-J, $\rightarrow$ Weidhase A, et al. $\mathbf{8 8 0}$

Gross AJ, $\rightarrow$ Jelkmann W 289

Gross M, Morisaki T, Pongratz D, Holmes EW, Zöllner N 1084

Grosser S, Kreymann G, Meierling St, Daerr W, Raedler A, Greten H 806

Grötz J, $\rightarrow$ Konermann M, et al. 1059

Grube E, $\rightarrow$ Schmieder R, et al. 256

Grünert J 757

Grunewald RW, $\rightarrow$ Kinne RK-H, et al. 199

Grupp C, $\rightarrow$ Kinne RK-H, et al. 199

Guder WG, Beck FX, Schmolke M 1091

Guggenmoos-Holzmann I, $\rightarrow$ Deininger $S$, et al. 906

Guggenmoos-Holzmann I, $\rightarrow$ Kiese M, et al. $\mathbf{1 0 8 2}$

Guggenmoos-Holzmann I, $\rightarrow$ Stark K, et al. 415

Guhlke U, $\rightarrow$ Rath W, et al. 768

Gurland HJ 757

Haas HG, Liebrich BM, Schaffner W 359

Habenicht A 1065

Habenicht AJR, Salbach P, Schettler G 53

Haberl R, Steinbeck G 744

Habersetzer R, $\rightarrow$ Scheidt $W$ von, et al. 241

Haegele $U, \rightarrow$ Reimers CD, et al. 335

Hagenmüller $\mathrm{F}, \rightarrow$ Lersch $\mathrm{C}$, et al. 523

Hahn D, $\rightarrow$ Zoller WG, et al. 830

Halimi P, $\rightarrow$ Sigal R, et al. 396
Hamann $\mathrm{K}, \rightarrow$ Barleben $\mathrm{H}$, et al. 518

Hammer C 430

Hančinová $D, \rightarrow$ Tajtáková $\mathrm{M}$, et al. 503

Handt S, $\rightarrow$ Kindler J, et al. 1042

Hänsch GM, $\rightarrow$ Blaas $P$, et al. 247

Haratz D, Manny N, Raz I 94

Harrer T, Wolf B, Kersten W, Schwartz W, Bergner D, Kalden JR 864

Harsch I, $\rightarrow$ Paschke R, et al. 942

Harthus H-P, $\rightarrow$ Jahn G, et al. 1003

Hartmann A, $\rightarrow$ Fric $M$, et al. 1189

Hartmann A, $\rightarrow$ Riedel R-R, et al. $\mathbf{5 0}$

Hartung $\mathrm{K}, \rightarrow$ Antes U, et al. 1066

Hauck R, Baur X $\mathbf{5 1 2}$

Haupt A, $\rightarrow$ Rath W, et al. 768

Hausen A, $\rightarrow$ Denz H, et al. 218

Hausen $A, \rightarrow$ Reibnegger $G$, et al. 43

Häusler $\mathrm{G}, \rightarrow$ Benker $\mathrm{G}$, et al. 1157

Hausser KH, Kalbitzer HR 396

Häussinger D 1096

Häussinger D, Steeb R, Gerok W 175

Häussinger $D, \rightarrow$ Lehmann $M$, et al. 873

Häußinger $\mathrm{K}, \rightarrow$ Dörfler $\mathrm{H}$, et al. 388

Hayakawa T, $\rightarrow$ Goto 1 , et al. 49

Hecht A, Lunzenauer K 331

Heering $\mathrm{P}, \rightarrow$ Plum $\mathrm{J}$, et al. 476

Heidbreder E, Bahner U, Hess M, Geiger H, Götz R, Kirsten R, Rascher W, Heidland A 1127

Heiden $W$ an der, $\rightarrow$ Nowotny B, et al. 964

Heidland $\mathrm{A}, \rightarrow$ Heidbreder E, et al. 1127

Heilmann L, Schmid-Schönbein $\mathrm{H} 559$

Heilmeyer L, $\rightarrow$ Cremer H-D, et al. 523

Heim J-M, Gottmann K, Weil J, Schiffl H, Lauster F, Loeschke K, Gerzer R 709

Heim J-M, $\rightarrow$ Karrenbrock B, et al. 213

Heine $\mathrm{H}, \rightarrow$ Barleben $\mathrm{H}$, et al. 518
Heinicke $\mathrm{A}, \rightarrow$ Eiff $\mathrm{M}$ von, et al. 372

Heinrich B, $\rightarrow$ Bogner JR, et al. 393, 1050

Heinz HP, $\rightarrow$ Antes U, et al. 1066

Hemmer ChJ, Kern P, Radtke KP, Egbring RE, Nawroth PP, Dietrich M 281

Henning HV, $\rightarrow$ Schrader J, et al. $\mathbf{7 7 4}$

Hensen J, Buhl M, Bähr V, Oelkers W $\mathbf{6 0 8}$

Henze Th, Prange HW, Talartschik J, Rumpf KW 1183

Herberhold C, $\rightarrow$ Fric $M$, et al. 1189

Herfarth C, $\rightarrow$ Rentchnick P, et al. 1194

Herken $\mathrm{H}, \rightarrow$ Born GVR, et al. 182

Hermanek P, $\rightarrow$ Spiessl B, et al. 984

Hermann E, $\rightarrow$ Mayet W-J, et al. 685

Hermans $\mathrm{H}, \rightarrow$ Zoller WG, et al. 830

Hermus A, $\rightarrow$ Hobma $S$, et al. 981

Herold $\mathrm{M}$, $\rightarrow$ Tilg $\mathrm{H}$, et al. 1083

Herrmann Ch, $\rightarrow$ Scholz KH, et al. 823

Herrmann M, $\rightarrow$ Krapf FE, et al. 299

Herzer P, $\rightarrow$ Schewe S, et al. 466

Herzog P, $\rightarrow$ Leopolder-Ochsendorf $A$, et al. 83

Hess B, Binswanger U 874

Hess $M, \rightarrow$ Heidbreder E, et al. 1127

Heydenreich F, $\rightarrow$ Weise F, et al. 26

Hinrichsen $\mathrm{H}, \rightarrow$ Linde $\mathrm{M}$, et al. 921

Hintze G $\mathbf{6 4 7}$

Hippéli $\mathrm{R}, \rightarrow$ Walker $\mathrm{S}$, et al. 187

Hirche $\mathrm{H}, \rightarrow$ Kraatz C, et al. 583

Hobma S, Hermus A, Pieters G, Smals A, Kloppenborg P 981

Hodenberg E von, $\rightarrow$ Feussner $G \mathbf{5 2 6}$

Hofferberth B, Brune GG 580

Hoffmann $\mathrm{H}, \rightarrow$ Spannagl M, et al. 901

Hoffmeister HM, Hörmann H-P, Beyer M, Seipel L 1178

Hoffmeister $\mathrm{H}, \rightarrow$ Kohlmeier L 454
Högl L, $\rightarrow$ Huhn C, et al. 853

Hollenbeck $\mathrm{M}, \rightarrow$ Plum $\mathrm{J}$, et al. 476

Holmes EW, $\rightarrow$ Gross M, et al. 1084

Holtermüller $\mathrm{KH}, \rightarrow$ Leopolder-Ochsendorf A, et al. 83

Holtmeier H-J, $\rightarrow$ Cremer H$\mathrm{D}$, et al. 523

Holubarsch $\mathrm{C}, \rightarrow$ Just $\mathrm{H}$, et al. 345

Holzer E, $\rightarrow$ Mayer W, et al. 431

Holzgreve $\mathrm{H}, \rightarrow$ Kormann BA, et al. 1027

Hombach V, $\rightarrow$ Schmidt A, et al. 286

Hombach V, $\rightarrow$ Wieshammer $S$, et al. 436

Hopf HC, $\rightarrow$ Schröder JM, et al. 345

Hopfenmüller W, $\rightarrow$ Weinke Th, et al. 277

Hörmann H-P, $\rightarrow$ Hoffmeister HM, et al. 1178

Horn A, Avenarius HJ, Deicher $\mathrm{H} \mathbf{4 6 0}$

Horn $\mathrm{K}, \rightarrow$ Spes $\mathrm{CH}$, et al. 872

Horowski R, $\rightarrow$ Wüster C, et al. 384

Horst H-J, $\rightarrow$ Zabel P, et al. 1217

Hötzel D, $\rightarrow$ Cremer H-D, et al. 523

Huang C 149

Huber $\mathrm{C}$, $\rightarrow$ Tilg $\mathrm{H}$, et al. 1083

Huber $\mathrm{H}, \rightarrow$ Denz $\mathrm{H}$, et al. 218

Huber I, $\rightarrow$ Scheidt W von, et al. 241

Hübner $\mathrm{G}, \rightarrow$ Reimers $\mathrm{CD}$, et al. 335

Huck K, $\rightarrow$ Schaaf L, et al. 1037

Huhn C, Nerl C, Siebert A, Högl L, Doering W, Kaboth W, Eichenlaub D $\mathbf{8 5 3}$

Husen $\mathrm{N}$ van, $\rightarrow$ Eiff $\mathrm{M}$ von, et al. 372

Ikenoue $\mathrm{H}, \rightarrow$ Yoshinari $\mathrm{M}$, et al. 441

Ivanyi $\mathrm{P} 773$

Jacob K, $\rightarrow$ Scheidt W von, et al. 241

Jahn G, Harthus H-P, Bröker $\mathrm{M}$, Borisch B, Platzer B, Plachter B 1003

Jahn HA, $\rightarrow$ Weidmann P, et al. 269 
Jakober B, Lingenfelser $T$, Glück H, Maassen T, Overkamp D, Renn W, Eggstein M 447

Janitschke $\mathrm{K}, \rightarrow$ Weinke Th, et al. 277

Jaresch S, Kley HK, Schlaghecke $\mathrm{R} 328$

Jaspers $\mathrm{C}, \rightarrow$ Benker $\mathrm{G}$, et al. 1157

Jelkmann W, Gross AJ 289

Jelkmann W, Wiedemann G 403

Jochum $M, \rightarrow$ Spannagl $M$, et al. 901

Jonge JPA de, $\rightarrow$ Riedel R-R, et al. $\mathbf{5 0}$

Jung $\mathrm{K}, \rightarrow$ Barleben $\mathrm{H}$, et al. 518

Junga G, $\rightarrow$ Franke-Raue K, et al. 597

Junge $\mathrm{E}, \rightarrow$ Eiff $\mathrm{M}$ von, et al. 372

Jungmann $\mathrm{E}, \rightarrow$ Eisenhauer $\mathrm{T}$, et al. $\mathbf{7 5 0}$

Junker L, $\rightarrow$ Barleben $\mathrm{H}$, et al. 518

Jürgens $\mathrm{U}, \rightarrow$ Baumgart $\mathrm{P}$, et al. $\mathbf{7 2 3}$

Jürgensen O 1049

Jurna I, Carlsson K-H, Kömen W, Bonke D 129

Just $\mathrm{H}$, Holubarsch C, Scholz H 345

Kaboth U, Rumpf KW, Lipp T, Bigge J, Nauck M, Beyer J-H, Seyde W, Kaboth W 18

Kaboth W, $\rightarrow$ Huhn C, et al. 853

Kaboth W, $\rightarrow$ Kaboth U, et al. 18

Kaffarnik $\mathrm{H}, \rightarrow$ Meyer JG, et al. 60

Kalbitzer HR, $\rightarrow$ Hausser KH 396

Kalden JR, $\rightarrow$ Harrer $T$, et al. 864

Kalden JR, $\rightarrow$ Krapf FE, et al. 299

Kammerhuber $\mathrm{F}, \rightarrow$ Lind $\mathrm{P}$, et al. 472

Kamps $\mathrm{B}, \rightarrow$ Kamradt $\mathrm{T}$, et al. 1203

Kamradt T, Niese D, Brackmann $\mathrm{HH}$, Schneweis KE, Kamps B, Loo B van Euler P 1203

Kandt M, $\rightarrow$ Schrader J, et al. 774

Karrenbrock B, Heim J-M, Gerzer R 213

Karsch K-R, $\rightarrow$ Dittmann H, et al. 263

Kauert B, Schoppek B, Clar- mann $\mathrm{M} v$, Felgenhauer $\mathrm{N}$ 96

Kaumeier S, $\rightarrow$ Nowotny B, et al. 964

Kaumeier S, $\rightarrow$ Paschke R, et al. 942

Keck E, $\rightarrow$ Leicht E, et al. 678

Keck FS, $\rightarrow$ Duntas L, et al. 1013

Kehrer G, Bretschneider HJ 223

Keller E, $\rightarrow$ Dobos GJ, et al. 976

Kern P, $\rightarrow$ Hemmer ChJ et al. $\mathbf{2 8 1}$

Kersten W, $\rightarrow$ Harrer $\mathrm{T}$, et al. 864

Khalaf A-N, $\rightarrow$ Lehmann M, et al. 873

Kienecker EW, Becker KW, Dick P 146

Kiese M, Lenz K, Guggenmoos-Holzmann I, Stark $\mathrm{K}$, Bienzle U 1082

Kieselbach F, Kotanko P 869

Kiess W, $\rightarrow$ Weimann E 985

Kindler J, Giani G, Handt S, Sieberth H-G 1042

Kinne RK-H, Grupp C, Grunewald RW 199

Kiparski A v, Frei D, Fierz W, Frei G, Uhlschmid G, Largiader F, Binswanger U 408

Kirch W, $\rightarrow$ Linde $\mathrm{M}$, et al. 921

Kirchner $\mathrm{H}, \rightarrow$ Atzpodien $\mathrm{J}$ 1

Kirsten R, $\rightarrow$ Heidbreder E et al. 1127

Kleber FX, $\rightarrow$ Mayer W, et al. 431

Klehr H-U, $\rightarrow$ Fric M, et al. 1189

Klein M, $\rightarrow$ Rosen A, et al. 537

Klein W, $\rightarrow$ Eber B, et al. 594

Kleinberger G, $\rightarrow$ Deutsch E, et al. 788

Kleinböhl D, $\rightarrow$ Nowotny B, et al. 964

Kley HK, $\rightarrow$ Jaresch S, et al. 328

Klima G, $\rightarrow$ Eber B, et al. 594

Kloppenborg P, $\rightarrow$ Hobma S, et al. 981

Klotz U, $\rightarrow$ Walker S, et al. 959

Klussmann R 984

Knauf H, Kölle EU, Mutschler E 692

Knauf H, Wenk E, Schölmerich J, Goerg KJ, Gerok W,
Leser HG, Mutschler E 545

Kniemeyer HW, Merckle R, Stühmeier K, Sandmann W 1208

Knüttgen $\mathrm{D}$, Weidemann $\mathrm{D}$, Doehn M 1168

Köbberling J, Pickardt CR 886

Koch EMW, $\rightarrow$ Brüggemann $\mathrm{G}$, et al. 116

Koch HH, $\rightarrow$ Mann H 629

Koch HH, $\rightarrow$ Mann K 627

Koehler $\mathrm{CO}, \rightarrow$ Brüggemann $\mathrm{G}$, et al. 116

Koehler $\mathrm{CO}, \rightarrow$ Kuhlwein A, et al. $\mathbf{1 0 7}$

Koenig W, $\rightarrow$ Schmidt A, et al. 286

Kohl FV, $\rightarrow$ Schmidt JA et al. 191

Köhler E 532

Kohlmeier L, Hoffmeister H 454

Kölle EU, $\rightarrow$ Knauf $\mathrm{H}$, et al. 692

Költringer $\mathrm{P}, \rightarrow$ Lind $\mathrm{P}$, et al. 472

Kömen W, $\rightarrow$ Jurna I, et al. 129

Konermann M, Grötz J, Sorge-Hädicke B, Sanner B 1059

König $\mathrm{M}, \rightarrow$ Vogelberg $\mathrm{KH}$, et al. $\mathbf{5 8 8}$

Koop H, $\rightarrow$ Lankisch PG, et al. 565

Kormann BA, Holzgreve $\mathrm{H}$, Wolff-Kormann PG, Riedel KG 1027

Köster O, $\rightarrow$ Robertz-Vaupel GM, et al. 1076

Köster W, $\rightarrow$ Söhngen D, et al. 1071

Kotanko P, $\rightarrow$ Kieselbach F 869

Kraatz C, Benker G, Weber $F$, Lüdecke $D$, Hirche $H$ Reinwein D $\mathbf{5 8 3}$

Kramer A, $\rightarrow$ Rath W, et al. 768

Krämer $\mathrm{A}, \rightarrow$ Reibnegger $\mathrm{G}$, et al. 43

Kramer HJ, Bäcker A, Krampitz $\mathrm{G}$, Meyer-Lehnert $\mathrm{H}$, Michel H 1112

Krampitz G, $\rightarrow$ Kramer $\mathrm{HJ}$ et al. 1112

Krapf FE, Herrmann M, Leitmann W, Schwartländer B, Kalden JR 299

Krause A, $\rightarrow$ Schmidt JA, et al. 191

Krause $\mathrm{U}, \rightarrow$ Weber $\mathrm{T}$, et al. 951

Kreiker C, $\rightarrow$ Zabel P, et al 1217
Kreuzer $\mathrm{H}, \rightarrow$ Schmidt $\mathrm{T}$, et al. 294

Kreuzer $\mathrm{H}, \rightarrow$ Scholz $\mathrm{KH}$, et al. 823

Kreymann G, $\rightarrow$ Grosser S, et al. 806

Kribben A, $\rightarrow$ Sharma AM, et al. 664

Kronberger-Schaffer E, $\rightarrow$ Eber B, et al. 594

Krück F 788

Krüger $\mathrm{H}, \rightarrow$ Schewe $\mathrm{S}$, et al. 466

Krüger $\mathrm{K}, \rightarrow$ Zöllner $\mathrm{N}$, et al. 739

Küffer $G, \rightarrow$ Dörfler $H$, et al. 388

Kuhls $\mathrm{S}, \rightarrow$ Wehling $\mathrm{M}$, et al. 71

Kuhlwein A, Meyer HJ, Koehler CO 107

Kühn HA, $\rightarrow$ Cremer H-D, et al. 523

Kuhn W, $\rightarrow$ Rath W, et al. 768

Kuhnle U, $\rightarrow$ Wehling $M$, et al. 71

Künau $\mathrm{J}, \rightarrow$ Cremer $\mathrm{H}-\mathrm{D}$, et al. 523

Kuntz BME, $\rightarrow$ Söhngen D, et al. 1071

Kuramoto K, $\rightarrow$ Yamanouchi $\mathrm{H}$, et al. 1173

Kuroda $\mathrm{T}, \rightarrow$ Yoshinari $\mathrm{M}$, et al. 441

Kurowski V, $\rightarrow$ Wood WG, et al. 324

Kusterer K, $\rightarrow$ Schaaf L, et al. 1037

Laggner AN, $\rightarrow$ Deutsch E, et al. 788

Landgraf $\mathrm{R}, \rightarrow$ Spes $\mathrm{CH}$, et al. 872

Langer H-J, $\rightarrow$ Leicht E, et al. 678

Langer M 475

Langer $P, \rightarrow$ Tajtáková $M$, et al. 503

Langsteger W, $\rightarrow$ Lind $\mathrm{P}$, et al. 472

Lankisch PG, BuschmannKaspari H, Otto J, Schröder K, Koop H $\mathbf{5 6 5}$

Largiader F, $\rightarrow$ Kiparski A v, et al. 408

Laso Guzmán FJ, GonzalezBuitrago JM, Vela R, Cava $F$, Castro $S$ de 183

Laue A, $\rightarrow$ Gröne H-J, et al. 758

Laukamm-Josten $U, \rightarrow$ Deininger $S$, et al. 906

Lauster $\mathrm{F}, \rightarrow$ Heim J-M, et al. 709

Lechleitner $\mathrm{P}, \rightarrow$ Tilg $\mathrm{H}$, et al. $\mathbf{1 0 8 3}$ 
Ledergerber B, $\rightarrow$ Opravil M, et al. 397

Leffler $\mathbf{J}, \rightarrow$ Nitsch $\mathrm{J}$, et al. 673

Lehmann $M$, Petersen $K G$, Khalaf A-N, Häussinger D, Büchner C, Sellinger M, Peter K 873

Leicht E, Biro G, Keck E, Langer H-J 678

Leitmann W, $\rightarrow$ Krapf FE, et al. 299

Lenz K, $\rightarrow$ Deutsch E, et al. 788

Lenz K, $\rightarrow$ Kiese M, et al. 1082

Leonhardt $\mathrm{A}, \rightarrow$ Bommer $\mathrm{M}$, et al. $\mathbf{5 5 2}$

Leopolder-Ochsendorf A, Ochsendorf FR, Tews KH, Milbradt R, Herzog P, Holtermüller KH 83

Lersch C, Gain T, Siemens $\mathrm{M} v$, Hagenmüller F, Classen M 523

Leser $\mathrm{HG}, \rightarrow$ Knauf $\mathrm{H}$, et al. 545

Lewis RE Jr, $\rightarrow$ Cruse JM 402

Liebrich BM, $\rightarrow$ Haas $\mathrm{HG}$, et al. 359

Lind P, Langsteger W, Költringer $P$, Eber $B$, Kammerhuber $F$, Smolle-Jüttner $F$, Eber O 472

Linde $M$, Hinrichsen $H$, Schubert S, Kirch W 921

Lingenfelser T, $\rightarrow$ Jakober B, et al. 447

Linke E, $\rightarrow$ Stein G, et al. 1150

Lipp T, $\rightarrow$ Kaboth U, et al. 18

Lippert TH $\mathbf{5 8 1}$

Loeffler M, $\rightarrow$ Diehl V, et al. 905

Loeschke K, $\rightarrow$ Heim J-M, et al. 709

Löhrs U, $\rightarrow$ Mißler U, et al. 342

Loo B van Euler $\mathrm{P}, \rightarrow \mathrm{Kam}-$ radt $T$, et al. 1203

Loo $\mathrm{J}$ van de, $\rightarrow$ Eiff $\mathrm{M}$ von, et al. 372

Loos $\mathrm{M}, \rightarrow$ Antes U, et al. 1066

Loos U, $\rightarrow$ Duntas L, et al. 1013

Lorenz J, Müller-Quernheim J, Castillo-Höfer C, Doboszýnska A, Ferlinz R 728

Löser Chr, Fölsch UR, Peiper H-J, Schuster R, Creutzfeldt W $\mathbf{7 8 0}$

Lotterer E, $\rightarrow$ Bircher J 475

Lovett DH, $\rightarrow$ Davies M, et al. 1145
Lüdecke D, $\rightarrow$ Kraatz C, et al. 583

Lüderitz B 1216

Lüderitz B, Antoni H 517

Lüderitz $B, \rightarrow$ Nitsch $J$, et al. 673

Ludwig $M$, Wetzig $H$, Sauer A, Vetter $H \mathbf{5 7 0}$

Lüning M, Felix R 1075

Lunzenauer $\mathrm{K}$, $\rightarrow$ Hecht A 331

Lütcke $\mathrm{A}, \rightarrow$ Schmidt JA, et al. 191

Lüthy $R, \rightarrow$ Opravil $M$, et al. 397

Lutz $\mathrm{H} 840$

Maassen T, $\rightarrow$ Jakober B, et al. 447

Maciejewski W, $\rightarrow$ Mayer W, et al. 431

Mack $\mathrm{M}, \rightarrow$ Mißler U, et al. 342

Mair J, $\rightarrow$ Tilg H, et al. 1083

Maldonado Martín A, $\rightarrow$ Ruiz Martínez $M$, et al. 507

Malinovský E, $\rightarrow$ Tajtáková $\mathrm{M}$, et al. $\mathbf{5 0 3}$

Mann H, Koch HH 629

Mann K 650

Mann K, Koch HH 627

Mannebach $\mathrm{H}, \rightarrow$ Bogunovic $\mathrm{N}$, et al. 532

Manns $\mathrm{M}, \rightarrow$ Mayet W-J, et al. 685

Manny $N, \rightarrow$ Haratz $D$, et al. 94

Margraf J, Schneider S 1202

Mariß G, $\rightarrow$ Schmidt JA, et al. 191

Markl A, $\rightarrow$ Scheidt W von, et al. 241

Marten $\mathrm{J}, \rightarrow$ Scholz $\mathrm{KH}$, et al. 823

Martin J, $\rightarrow$ Davies M, et al. 1145

Matter L, $\rightarrow$ Stupp R, et al. 971

Matuschke A, $\rightarrow$ Bogner JR, et al. 393, 1050

Mauerberger A, $\rightarrow$ Wagner G 1156

Mayer $\mathrm{P}, \rightarrow$ Vogelberg $\mathrm{KH}$, et al. $\mathbf{5 8 8}$

Mayer W, Kleber FX, Wilske B, Preac-Mursic V, Maciejewski W, Sigl H, Holzer E 431

Mayet W-J, Hermann E, Bachmann M, Manns M, Meyer zum Büschenfelde K-H 685

Mehnert $\mathrm{H}, \rightarrow$ Stiegler $\mathrm{H}$, et al. $\mathbf{3 8 0}$

Meierling St, $\rightarrow$ Grosser S, et al. 806
Meier-Ruge W 453

Meinke J, $\rightarrow$ Walker S, et al. 959

Merckle R, $\rightarrow$ Kniemeyer HW, et al. 1208

Mertins L, $\rightarrow$ Brockmeyer NH, et al. 1229

Meske S, $\rightarrow$ Dobos GJ, et al. 976

Meyer E, $\rightarrow$ Stark K, et al. 415

Meyer $\mathrm{HJ}, \rightarrow$ Kuhlwein A, et al. $\quad 107$

Meyer JG, Renczes JG, Kaffarnik $\mathrm{H} \quad \mathbf{6 0}$

Meyer zum Büschenfelde K$\mathrm{H}, \rightarrow$ Mayet W-J, et al. 685

Meyer-Lehnert $\mathrm{H}, \rightarrow$ Kramer $\mathrm{HJ}$, et al. 1112

Michel $\mathrm{H}, \rightarrow$ Kramer $\mathrm{HJ}$, et al. 1112

Middeke $\mathrm{M}, \rightarrow$ Bogner JR, et al. 1032

Middeke $\mathrm{M}, \rightarrow$ Zoller WG, et al. 830

Miehle W, $\rightarrow$ Fehr K, et al. 453

Mihatsch MJ, $\rightarrow$ Fuhrer JA, et al. 1198

Mihatsch MJ, $\rightarrow$ Stupp R, et al. 971

Milbradt R, $\rightarrow$ LeopolderOchsendorf A, et al. 83

Miñarro del Moral $\mathrm{J}$, $\rightarrow$ Garcia Ontiveros A, et al. 496

Mißler U, Mack M, Nowak G, Müller-Esch G, Reusche E, Borgis K-J, Löhrs U, Arnold $\mathrm{H} \quad 342$

Mitchner J, $\rightarrow$ Baumgartner FJ, et al. 485

Möller G 32, 212, 217, 440, 905, 1075, 1177

Moore TC, $\rightarrow$ Baumgartner FJ, et al. 485

Moreno Abadía V, $\rightarrow$ Ruiz Martínez M, et al. $\mathbf{5 0 7}$

Mori T, $\rightarrow$ Schlondorff D 1138

Morisaki T, $\rightarrow$ Gross M, et al. 1084

Moser R, $\rightarrow$ Christen RD, et al. 427

Mudra $\mathrm{H}, \rightarrow$ Spes $\mathrm{CH}$, et al. 872

Müller F, Seifert O 453

Müller HE 240

Müller P, Schlimok G, Gerber H, Renner D 332

Müller $\mathrm{R}, \rightarrow$ Deininger $\mathrm{S}$, et al. 906

Müller R, $\rightarrow$ Stark K, et al. 415

Müller Th, $\rightarrow$ Oster $\mathrm{P}$, et al. 421
Müller-Esch G, $\rightarrow$ Mißler U, et al. 342

Müller-Klieser $\mathrm{W}, \rightarrow$ Wiedemann $G$, et al. 33

Müller-Quernheim J, $\rightarrow$ Lorenz J, et al. 728

Munker R, $\rightarrow$ Schleuning M 841

Mutschler $\mathrm{E}, \rightarrow$ Knauf $\mathrm{H}$, et al. 545, 692

Naber $\mathrm{D}, \rightarrow$ Bommer $\mathrm{M}$, et al. 552

Nachbaur D, $\rightarrow$ Denz $H$, et al. 218

Naegele $M, \rightarrow$ Reimers $C D$, et al. 335

Nasemann T, Sauerbrey W 487

Nauck $M, \rightarrow$ Kaboth U, et al. 18

Nawroth PP, $\rightarrow$ Hemmer ChJ, et al. 281

Nayler WG 984

Neftel KA, $\rightarrow$ Christen RD, et al. 427

Neftel KA, $\rightarrow$ Schindler JM 237

Nerl C, $\rightarrow$ Bogner JR, et al. 393

Nerl C, $\rightarrow$ Huhn C, et al. 853

Neu U, $\rightarrow$ Schrader J, et al. 774

Neuhaus KL, $\rightarrow$ Scholz KH, et al. 823

Niese $\mathrm{D}, \rightarrow$ Kamradt $\mathrm{T}$, et al. 1203

Ninnemann JL, $\rightarrow$ Faist E, et al. 1202

Nitsch J, Leffler J, Lüderitz B 673

Nordestgaard AG, $\rightarrow$ Baumgartner FJ, et al. 619

Nowak G, $\rightarrow$ Mißler U, et al. 342

Nowotny B, Teuber J, Heiden W an der, Schlote B, Kleinböhl D, Schmidt R, Kaumeier S, Usadel KH 964

Oberleithner H 1087

Obernosterer A, $\rightarrow$ Eber B, et al. 594

Ochsendorf FR, $\rightarrow$ Leopolder-Ochsendorf A, et al. 83

Oelkers W, $\rightarrow$ Hensen J, et al. 608

Öfner D, $\rightarrow$ Sandbichler P, et al. 290

Ohlmeier $\mathrm{H}, \rightarrow$ Bogunovic $\mathrm{N}$, et al. 532

Okamura $\mathrm{K}, \rightarrow$ Yoshinari $\mathrm{M}$, et al. 441

Olek K, $\rightarrow$ Poller W, et al. 857 
Ollenschläger $\mathrm{G}, \rightarrow$ Albus $\mathrm{C}$, et al. 77

Opravil M, Weber R, Ledergerber B, Speich R, Siegenthaler W, Lüthy R 397

Oster P, Müller Th, SchmidtGayk H, Schlierf G $\mathbf{4 2 1}$

Otte $\mathrm{J}, \rightarrow$ Wees $\mathrm{J}$ van, et al. 1054

Otto J, $\rightarrow$ Lankisch PG, et al. 565

Overkamp D, $\rightarrow$ Jakober B, et al. 447

Paschke R, Harsch I, Schlote B, Vardarli I, Schaaf L, Kaumeier S, Teuber J, Usadel KH 942

Paschke R, $\rightarrow$ Schaaf L, et al. 1037

Paulley JW, Pelser HE 341

Peiper $\mathrm{H}-\mathrm{J}, \rightarrow$ Löser $\mathrm{Chr}$, et al. $\mathbf{7 8 0}$

Pelser HE, $\rightarrow$ Paulley JW 341

Permanetter W, $\rightarrow$ Dörfler H, et al. 388

Pernthaler $\mathrm{H}, \rightarrow$ Sandbichler $P$, et al. 290

Peter $\mathrm{HH}, \rightarrow$ Blaas $\mathrm{P}$, et al. 247

Peter HH, $\rightarrow$ Dobos GJ, et al. 976

Peter K, $\rightarrow$ Lehmann $M$, et al. 873

Peters R, $\rightarrow$ Albus C, et al. 77

Petersen KG, $\rightarrow$ Lehmann M, et al. 873

Pfeifer $\mathrm{U}, \rightarrow$ Fric $\mathrm{M}$, et al. 1189

Pfeiffer EF, $\rightarrow$ Duntas L, et al. 1013

Pfeilschifter J 1134

Pfreundschuh $\mathrm{M}, \rightarrow$ Diehl V, et al. 905

Pickardt CR, $\rightarrow$ Köbberling J 886

Pickardt $\mathrm{R}, \rightarrow$ Bommer $\mathrm{M}$, et al. 552

Pieters G, $\rightarrow$ Hobma S, et al. 981

Pizzulii L, $\rightarrow$ Robertz-Vaupel GM, et al. $\mathbf{1 0 7 6}$

Plachter B, $\rightarrow$ Jahn G, et al. 1003

Platt D 396, 1194

Platzer B, $\rightarrow$ Jahn G, et al. 1003

Plum J, Hollenbeck M, Heering $P$, Grabensee B 476

Pohle HD, $\rightarrow$ Weinke Th, et al. 533

Poller W, Faber J-P, Olek K 857

Pongratz DE, $\rightarrow$ Reimers $\mathrm{CD}$, et al. 335
Pongratz D, $\rightarrow$ Gross $M$, et al. 1084

Prange HW, $\rightarrow$ Henze Th, et al. 1183

Preac-Mursic V, $\rightarrow$ Mayer W, et al. 431

Purucker E, Wernze H 1008

Quellhorst E $\mathbf{7 4 3}$

Radtke $\mathrm{KP}, \rightarrow$ Hemmer $\mathrm{ChJ}$, et al. 281

Raedler A, $\rightarrow$ Grosser S, et al. 806

Raedsch R, Walter-Sack I, Weber E, Blessing J $\mathbf{4 8 8}$

Rahn $\mathrm{KH}, \rightarrow$ Baumgart $\mathrm{P}$ 320

Rahn $\mathrm{KH}, \rightarrow$ Baumgart $\mathrm{P}$ et al. $\mathbf{7 2 3}$

Rahn KH, $\rightarrow$ Bönner G 48 , 511

Rascher W, $\rightarrow$ Heidbreder E et al. 1127

Rath W, Schrader J, Guhlke $\mathrm{U}$, Buhr-Schinner $\mathrm{H}$, Haupt A, Kramer A, Kuhn W 768

Rath W, $\rightarrow$ Schrader J, et al. 774

Raue F, $\rightarrow$ Franke-Raue K, et al. 597

Raz I, $\rightarrow$ Haratz D, et al. 94

Redel DA 536

Reibnegger G, Fuchs D, Goedert JJ, Hausen A, Krämer A, Werner ER, WernerFelmayer G, Dierich MP, Wachter $\mathrm{H} \mathbf{4 3}$

Reibnegger G, $\rightarrow$ Denz. $\mathrm{H}$, et al. 218

Reif M 1182

Reimann $\mathrm{H}, \rightarrow$ Barleben $\mathrm{H}$ et al. 518

Reimers CD, Naegele $M$, Hübner $G$, Haegele $U$, Wagner St, Pongratz DE 335

Reinhart K, Eyrich K 525

Reinwein $\mathrm{D}, \rightarrow$ Benker $\mathrm{G}$, et al. 1157

Reinwein D, $\rightarrow$ Kraatz C, et al. $\mathbf{5 8 3}$

Renczes JG, $\rightarrow$ Meyer JG et al. 60

Renn W, $\rightarrow$ Jakober B, et al. 447

Renner D, $\rightarrow$ Müller $P$, et al. 332

Rentchnick P, Herfarth C, Senn H-J 1194

Rett $\mathrm{K}, \rightarrow$ Stiegler $\mathrm{H}$, et al. 380

Reusche E, $\rightarrow$ Mißler $U$ et al. 342

Richter K 297
Ridderskamp P, Schlaghecke R 927

Rieckmann P, Weber T, Felgenhauer $\mathrm{K} 12$

Riedel $\mathrm{KG}, \rightarrow$ Kormann BA, et al. 1027

Riedel R-R, Schmitt A, Jonge JPA de, Hartmann A $\mathbf{5 0}$

Riegel W, $\rightarrow$ Dobos GJ, et al. 976

Riesen W, $\rightarrow$ Weidmann P, et al. 269

Ritz E, $\rightarrow$ Stein G, et al. 1150

Ritz R, $\rightarrow$ Deutsch E, et al 788

Rob PM, Fandrey J 835

Robertz-Vaupel GM, Glasmacher AG, Vogel J, Vaupel HA, Köster O, Pizzulii L 1076

Roos N, $\rightarrow$ Eiff $\mathrm{M}$ von, et al. 372

Rosen A, Klein M, Beck A 537

Rosenthal $\mathrm{J}, \rightarrow$ Duntas L, et al. 1013

Rubin E, Damjanov I 840

Rüddel $\mathrm{H}, \rightarrow$ Schmieder $\mathrm{R}$ et al. 256

Ruiz Martínez M, Gil Extremera B, Maldonado Martín A, Cantero Hinojosa $\mathbf{J}$ Moreno Abadía V $\mathbf{5 0 7}$

Rumpelt $\mathrm{HJ}, \rightarrow$ Thoenes W, et al. 1102

Rumpf KW, $\rightarrow$ Henze Th, et al. 1183

Rumpf KW, $\rightarrow$ Kaboth U, et al. 18

Runge $\mathrm{U}, \rightarrow$ Weise $\mathrm{F}$, et al. 26

Ruschitzka F, $\rightarrow$ Schrader J, et al. 774

Säger H-D, $\rightarrow$ Schaaf L, et al. 1037

Salbach $\mathrm{P}, \rightarrow$ Habenicht AJR, et al. 53

Salzberger B, $\rightarrow$ Fätkenheuer $G$, et al. 89

Sandbichler $\mathrm{P}$, Pernthaler $\mathrm{H}$ Schmid Th, Öfner D, Aigner F, Tötsch $M$, Schmid KW 290

Sandkühler J, $\rightarrow$ Fu Q-G, et al. 125

Sandmann W, $\rightarrow$ Kniemeyer HW, et al. 1208

Sanner B $\rightarrow$ Konermann M, et al. 1059

Sato $\mathrm{K}, \rightarrow$ Yoshinari $\mathrm{M}$, et al. 441

Sauer A, $\rightarrow$ Ludwig M, et al. 570

Sauerbrey W, $\rightarrow$ Nasemann T 487
Schaaf L, Gresclner $M$, Geissler W, Ecken B, Seif FJ, Usadel KH $6 \mathbf{9}$

Schaaf L, Grescher M, Paschke R, Kuserer K, Teuber J, Huck K.Schmidt $\mathrm{R}$, Säger H-D, Usidel KH 1037

Schaaf L, $\rightarrow$ Pasthke R, et al. 942

Schaefer K, $\rightarrow$ Stein $\mathrm{F}_{\mathrm{s}}$, et al. 1150

Schäfer HM, $\rightarrow$ Albus C, et al. 77

Schaffner W, $\rightarrow$ Hias HG, et al. 359

Scharf RE, $\rightarrow$ Wehneier A, et al. $\mathbf{8 4 7}$

Schattenfroh S, $\rightarrow$ Sharma AM, et al. 664

Schattenkirchner $\mathrm{M}, \rightarrow$ Fehr $\mathrm{K}$, et al. 453

Schatz H 635

Scheidt W von, Böım $M$, Huber I, Habersetz:r R, Jacob K, Markl A, Autenrieth $G 241$

Scheler F, $\rightarrow$ Schrider J, et al. 1119

Scherberich $\mathrm{J}, \rightarrow$ Eis'nhauer $\mathrm{T}$, et al. 750

Scherer W, $\rightarrow$ Weirke Th, et al. 533

Schettler G, $\rightarrow$ Hibenicht AJR, et al. 53

Scheurlen PG 296

Schewe $S$, Herzer P, Krüger $\mathrm{H} \quad 466$

Schiffl H 718

Schiffl $\mathrm{H}, \rightarrow$ Heir $\mathrm{J}-\mathrm{M}$, et al. 709

Schiff $\mathrm{H}, \rightarrow$ Spannigl $M$, et al. 901

Schild HH, Schwed:n F 435

Schindler JM, Nefte KA 237

Schlaak M, $\rightarrow$ Zabel P.et al. 1217

Schlaghecke R, $\rightarrow$ Jaresch S, et al. 328

Schlaghecke $\mathrm{R}, \rightarrow$ Fidderskamp P 927

Schlebusch $\mathrm{H}, \rightarrow$ Schnieder $\mathrm{R}$, et al. 256

Schleinkofer L, $\rightarrow$ Viedemann $G$, et al. 33

Schleuning $M$, Munksr $R$ 841

Schlierf G, $\rightarrow$ Oster P, et al. 421

Schlimok $G, \rightarrow$ Müler $P$ et al. 332

Schlondorff D, Mori T 1138

Schlossberg D 886

Schlote B, $\rightarrow$ Nowotiy B, et al. 964 
Schlote B, $\rightarrow$ Paschke R, et al. 942

Schlup $\mathrm{R}, \rightarrow$ Christen RD, et al. 427

Schmelzle $\mathrm{A}, \rightarrow$ Wüster $\mathrm{C}$, et al. 384

Schmid KW, $\rightarrow$ Sandbichler $P$, et al. 290

Schmid Th, $\rightarrow$ Sandbichler P, et al. 290

Schmid-Schönbein $\mathrm{H}, \quad \rightarrow$ Heilmann L $\mathbf{5 5 9}$

Schmidt A, Bunjes D, Friedrich J, Koenig W, Eggeling $T$, Hombach V 286

Schmidt JA, Krause A, Feddersen $\mathrm{CO}$, Kohl FV, Mariß G, Lütcke A, Wichert $P$ von 191

Schmidt $\mathrm{R}, \rightarrow$ Nowotny $\mathrm{B}$, et al. 964

Schmidt R, $\rightarrow$ Schaaf L, et al. 1037

Schmidt T, Tebbe U, Schrader J, Brune S, Kreuzer H 294

Schmidt-Gayk $\mathrm{H}, \rightarrow$ Oster $\mathrm{P}$, et al. 421

Schmieder R, Grube E, Rüddel $H$, Schlebusch $H$, Schulte W 256

Schmitt A, $\rightarrow$ Riedel R-R, et al. 50

Schmittenbecher PP, Endres W 346

Schmolke M, $\rightarrow$ Guder WG, et al. 1091

Schneider A, $\rightarrow$ Stein G, et al. 1150

Schneider S, $\rightarrow$ Margraf J 1202

Schneider T $\mathbf{5 7 6}$

Schneider $\mathrm{W}, \rightarrow$ Söhngen $\mathrm{D}$, et al. 1071

Schneider W, $\rightarrow$ Thomas M, et al. 539

Schneider W, $\rightarrow$ Wehmeier A, et al. 847

Schneweis KE, $\rightarrow$ Kamradt $T$, et al. 1203

Schoel G, $\rightarrow$ Schrader J, et al. 774, 1119

Schohn DC, $\rightarrow$ Weidmann P, et al. 269

Schollmeyer P, $\rightarrow$ Ballé C 38

Schollmeyer P, $\rightarrow$ Dobos GJ, et al. 976

Schölmerich $\mathrm{J}, \rightarrow$ Knauf $\mathrm{H}$, et al. 545

Schölmerich P, $\rightarrow$ Schuster HP, et al. 1182

Scholz A, $\rightarrow$ Wüster C, et al. 384

Scholz H, $\rightarrow$ Just $\mathrm{H}$, et al. 345

Scholz KH, Marten J, Herrmann Ch, Spaar U, Tebbe
U, Neuhaus KL, Kreuzer H 823

Schönborn $\mathrm{H}, \rightarrow$ Schuster HP, et al. 1182

Schoppek B, $\rightarrow$ Kauert B, et al. 96

Schrader J, Schoel G, Scheler F 1119

Schrader J, Tebbe U, Borries M, Ruschitzka F, Schoel G, Kandt M, Warneke G, Züchner $\mathrm{C}$, Weber $\mathrm{MH}$, Neu U, Rath W, Henning HV 774

Schrader J, $\rightarrow$ Rath W, et al. 768

Schrader J, $\rightarrow$ Schmidt T, et al. 294

Schrappe-Bächer M, $\rightarrow$ Fätkenheuer $\mathrm{G}$, et al. 89

Schreiber MA, $\rightarrow$ Bogner JR, et al. 393

Schröder JM, Hopf HC, Wagner $\mathrm{G}$, Amelung $\mathrm{F}$ 345

Schröder K, $\rightarrow$ Lankisch PG, et al. 565

Schubert $\mathrm{S}, \rightarrow$ Linde $M$, et al. 921

Schuff-Werner $\mathrm{P}, \rightarrow$ Weidhase A, et al. $\mathbf{8 8 0}$

Schulte W, $\rightarrow$ Schmieder R et al. 256

Schultes U, $\rightarrow$ Weinke Th, et al. 277

Schultze JL, $\rightarrow$ Stettin A, et al. 896

Schulz $G, \rightarrow$ Weber $T$, et al. 951

Schüpbach J 32

Schuster HP, Schölmerich P, Schönborn $\mathrm{H}$, Baum $\mathrm{P}$ 1182

Schuster HP, $\rightarrow$ Deutsch E et al. 788

Schuster $\mathrm{R}$, $\rightarrow$ Löser $\mathrm{Chr}$, et al. $\mathbf{7 8 0}$

Schwartländer $\mathrm{B}, \rightarrow \mathrm{Krapf}$ FE, et al. 299

Schwartz A, $\rightarrow$ Born GVR, et al. 182

Schwartz W, $\rightarrow$ Harrer T, et al. 864

Schweden F, $\rightarrow$ Schild $\mathrm{HH}$ 435

Schwinger RHG, Böhm M, Erdman E 797

Schwinger RHG, $\rightarrow$ Böhm $\mathrm{M}$, et al. $\mathbf{8 8 7}$

Seibold $\mathrm{H}, \rightarrow$ Wieshammer S, et al. 436

Seidel D, $\rightarrow$ Thiery J, et al. 814

Seidl S, $\rightarrow$ Spielmann W 1156

Seif FJ, $\rightarrow$ Schaaf L, et al. 669
Seifert O, $\rightarrow$ Müller F 453

Seipel L, $\rightarrow$ Dittmann $H$, et al. 263

Seipel L, $\rightarrow$ Hoffmeister HM, et al. 1178

Sellinger $\mathrm{M}, \rightarrow$ Lehmann $\mathrm{M}$, et al. 873

Senn H-J, $\rightarrow$ Rentchnick P, et al. 1194

Seyde W, $\rightarrow$ Kaboth U, et al. 18

Seyferth W, $\rightarrow$ Zeitler E 840

Sharma AM, Arntz H-R, Kribben A, Schattenfroh S Distler A 664

Sharma SK, $\rightarrow$ Dakshinamurti $\mathrm{K}$, et al. 142

Shaw SG, $\rightarrow$ Weidmann P, et al. 269

Shimada $\mathrm{H}, \rightarrow$ Yamanouchi $\mathrm{H}$, et al. 1173

Siebert A, $\rightarrow$ Huhn C, et al. 853

Sieberth $\mathrm{H}-\mathrm{G}, \rightarrow$ Kindler J, et al. 1042

Siegel EG 306

Siegenthaler W, $\rightarrow$ Opravil $\mathrm{M}$, et al. 397

Siemens $\mathrm{Mv}, \rightarrow$ Lersch $\mathrm{C}$, et al. 523

Sigal R, Doyon D, Halimi P, Atlan H 396

Sigl H, $\rightarrow$ Mayer W, et al. 431

Simon C, Stille W 522

Smals A, $\rightarrow$ Hobma S, et al. 981

Smolle-Jüttner $\mathrm{F}, \rightarrow$ Lind $\mathrm{P}$, et al. 472

Söhngen D, Köster W, Kuntz BME, Glück S, Schneider W 1071

Sonnenberg E van 25

Sorg M, $\rightarrow$ Zöllner N, et al. 739

Sorge-Hädicke B, $\rightarrow$ Konermann $\mathrm{M}$, et al. $\mathbf{1 0 5 9}$

Spaar U, $\rightarrow$ Scholz KH, et al. 823

Spagnolo $\mathrm{N}, \rightarrow$ Fontana $\mathrm{F}$, et al. 313

Spannagl M, Schiffl H, Hoffmann $\mathrm{H}$, Jochum $M 901$

Spanuth E, $\rightarrow$ Vogel G $\mathbf{1 0 2 0}$

Speich R, $\rightarrow$ Opravil M, et al. 397

Spengel FA, $\rightarrow$ StautnerBrückmann C 1195

Spes $\mathrm{CH}$, Angermann CE, Horn K, Strasser T, Mudra $\mathrm{H}$, Landgraf $\mathrm{R}$, Theisen $\mathrm{K}$ 872

Spielmann W, Seidl S 1156

Spiessl B, Beahrs OH, Hermanek P 984

Spüler M, $\rightarrow$ Dimpfel W, et al. 136
Stark K, Müller R, Guggenmoos-Holzmann I, Deininger S, Meyer E, Bienzle U 415

Stark K, $\rightarrow$ Kiese M, et al. 1082

Stautner-Brückmann $\quad$ C Spengel FA 1195

Stechemesser E, $\rightarrow$ Stettin A, et al. 896

Steeb R, $\rightarrow$ Häussinger D, et al. 175

Steimann $\mathrm{R}$, $\rightarrow$ Eiff $\mathrm{M}$ von, et al. 372

Stein G, Schneider A, Toß H, Linke E, Schaefer K, Ritz E 1150

Steinbeck G, $\rightarrow$ Haberl R 744

Steinhausen $\mathrm{M}, \rightarrow$ Zimmerhackl LB, et al. 166

Stettin A, Schultze JL, Stechemesser E, Berg PA 896

Stiegler $\mathrm{H}$, Wicklmayr $\mathrm{M}$, Rett K, Mehnert H $\mathbf{3 8 0}$

Stille W, $\rightarrow$ Simon C 522

Störkel S, $\rightarrow$ Thoenes W, et al. 1102

Strasser T, $\rightarrow$ Spes $\mathrm{CH}$, et al. 872

Streuli RA, $\rightarrow$ Fuhrer JA, et al. 1198

Streuli RA, $\rightarrow$ Stupp R, et al. 971

Struve C 402

Stühmeier K, $\rightarrow$ Kniemeyer HW, et al. 1208

Stupp R, Mihatsch MJ, Matter L, Streuli RA 971

Tajták J, $\rightarrow$ Tajtáková $\mathrm{M}$, et al. $\mathbf{5 0 3}$

Tajtáková M, Hančinová D, Langer P, Tajták J, Földes O, Malinovský E, Varga J 503

Talartschik $\mathrm{J}, \rightarrow$ Eisenhauer $\mathrm{T}$, et al. $\mathbf{7 5 0}$

Talartschik $\mathrm{J}, \rightarrow$ Henze Th, et al. 1183

Tebbe $\mathrm{U}, \rightarrow$ Schmidt $\mathrm{T}$, et al. 294

Tebbe $\mathrm{U}, \rightarrow$ Scholz KH, et al. 823

Tebbe U, $\rightarrow$ Schrader J, et al. 774

Tegtmeyer F-K, $\rightarrow$ Wees J van, et al. 1054

Teuber J, $\rightarrow$ Nowotny B, et al. 964

Teuber J, $\rightarrow$ Paschke R, et al. 942

Teuber J, $\rightarrow$ Schaaf L, et al. 1037

Tews KH, $\rightarrow$ Leopolder-Ochsendorf $\mathrm{A}$, et al. 83 
Thaler $\mathrm{J}, \rightarrow$ Denz $\mathrm{H}$, et al. 218

Theisen $\mathrm{K}, \rightarrow$ Spes $\mathrm{CH}$, et al. 872

Theisen $\mathrm{K}, \rightarrow$ Wehling $\mathrm{M}$, et al. 71

Thiery J, Creutzfeldt C, Creutzfeldt W, Walli AK, Seidel D 814

Thoenes W, Rumpelt HJ, Störkel S 1102

Thomas GJ, $\rightarrow$ Davies M, et al. 1145

Thomas M, Gattermann N, Schneider W 539

Thomas W, $\rightarrow$ Albus C, et al. 77

Tilg H, Mair J, Herold M, Aulitzky WE, Lechleitner P, Dienstl F, Huber C 1083

Tillmann $\mathrm{K}, \rightarrow$ Fehr $\mathrm{K}$, et al. 453

Tölle R, Buchkremer G 430

Toporsch M, $\rightarrow$ Eber B, et al. 594

Toß H, $\rightarrow$ Stein G, et al. 1150

Tötsch $\mathrm{M}, \rightarrow$ Sandbichler $\mathrm{P}$, et al. 290

Tracy S, $\rightarrow$ Wiegand V, et al. 914

Treiber G, $\rightarrow$ Walker $\mathrm{S}$, et al. 959

Uhlschmid G, $\rightarrow$ Kiparski A v, et al. 408

Unterberg $C$, $\rightarrow$ Weidhase A, et al. $\mathbf{8 8 0}$

Usadel KH, $\rightarrow$ Nowotny B, et al. 964

Usadel $\mathrm{KH}, \rightarrow$ Paschke $\mathrm{R}$, et al. 942

Usadel $\mathrm{KH}, \rightarrow$ Schaaf L, et al. 669, 1037

Vaith P, $\rightarrow$ Dobos GJ, et al. 976

Vardarli I, $\rightarrow$ Paschke R, et al. 942

Varga $\mathrm{J}, \rightarrow$ Tajtáková $\mathrm{M}$, et al. $\mathbf{5 0 3}$

Vaughan Williams EM 1026

Vaupel HA, $\rightarrow$ Robertz-Vaupel GM, et al. 1076

Vecsei P, $\rightarrow$ Franke-Raue K, et al. 597

Vela $\mathrm{R}, \rightarrow$ Laso Guzmán FJ, et al. $\mathbf{1 8 3}$

Vetter $\mathrm{H}, \rightarrow$ Ludwig $\mathrm{M}$, et al. 570

Voelker W, $\rightarrow$ Dittmann $\mathrm{H}$, et al. 263

Vogel G, Spanuth E 1020

Vogel J, $\rightarrow$ Robertz-Vaupel GM, et al. 1076

Vogelaers D, $\rightarrow$ Wilde V de, et al. 615
Vogelberg KH, Mayer P, König $M \mathbf{5 8 8}$

Vollmar AM 699

Wacher $\mathrm{H}, \rightarrow$ Denz $\mathrm{H}$, et al. 218

Wachter $\mathrm{H}, \rightarrow$ Reibnegger $\mathrm{G}$, et al. 43

Wackenheim A, Badoz A 773

Wagenknecht $\mathrm{C}$, $\rightarrow$ Barleben $\mathrm{H}$, et al. $\mathbf{5 1 8}$

Wagner G 190

Wagner $G$, Mauerberger A 1156

Wagner $\mathrm{G}, \rightarrow$ Schröder JM, et al. 345

Wagner St, $\rightarrow$ Reimers CD, et al. 335

Walenta $\mathrm{S}, \rightarrow$ Wiedemann G, et al. 33

Walger $\mathrm{P}, \rightarrow$ Baumgart $\mathrm{P}$, et al. 723

Walger $\mathrm{P}, \rightarrow$ Eiff $\mathrm{M}$ von, et al. 372

Walker S, Hippéli R, Goës R 187

Walker S, Meinke J, Klotz U, Treiber G, Bode JC 959

Walli AK, $\rightarrow$ Thiery J, et al. 814

Walter-Sack I, $\rightarrow$ Raedsch R, et al. 488

Walter-Sack I, $\rightarrow$ Weber E 932

Walter-Sack I, $\rightarrow$ Zöllner N, et al. 101

Warneboldt D, $\rightarrow$ Eisenhauer $\mathrm{T}$, et al. $\mathbf{7 5 0}$

Warneke $\mathrm{G}, \rightarrow$ Schrader J, et al. 774

Weber B 537

Weber E 1085

Weber E, Walter-Sack I 932

Weber E, $\rightarrow$ Raedsch R, et al. 488

Weber F, $\rightarrow$ Kraatz C, et al. 583

Weber $\mathrm{G}, \rightarrow$ Weinke Th et al. 277

Weber L, $\rightarrow$ Wieshammer S, et al. 436

Weber MH, $\rightarrow$ Schrader J, et al. 774

Weber R, $\rightarrow$ Opravil M, et al. 397

Weber S, $\rightarrow$ Blaas $\mathrm{P}$, et al. 247

Weber T, Schulz G, Beyer J, Geiling $\mathrm{H}$, Cordes U, Diederich $\mathrm{C}$, Krause U 951

Weber $\mathrm{T}$, $\rightarrow$ Rieckmann $\mathrm{P}$, et al. 12

Wees J van, Tegtmeyer F-K, Otte J, Wood WG, Braun J 1054
Wehling M, Kuhls S, Kuhnle $\mathrm{U}$, Theisen $\mathrm{K} \mathbf{7 1}$

Wehmeier A, Scharf RE, Schneider W $\mathbf{8 4 7}$

Wehr M, $\rightarrow$ Benker G, et al. 631

Wehrmann T, Caspary WF 602

Weidemann $\mathrm{D}, \rightarrow$ Knüttgen D, et al. 1168

Weidhase A, Gröne H-J, Unterberg C, Schuff-Werner $\mathrm{P}$, Wiegand $\mathrm{V} \mathbf{8 8 0}$

Weidmann P, Schohn DC, Riesen W, Jahn HA, Ferrari $P$, Shaw SG, BerettaPiccoli C 269

Weil J, $\rightarrow$ Heim J-M, et al. 709

Weimann E, Kiess W 985

Weinke Th, Scherer W, Pohle HD 533

Weinke Th, Weber G, Schultes U, Hopfenmüller W, Janitschke K 277

Weise F, Heydenreich F, Gehrig W, Runge U 26

Weismüller $\mathrm{P}$, $\rightarrow$ Wieshammer S, et al. 436

Wenger R, Brandstetter BM 190

Wenk E, $\rightarrow$ Knauf $\mathrm{H}$, et al. 545

Werner ER, $\rightarrow$ Denz $\mathrm{H}$, et al. 218

Werner ER, $\rightarrow$ Reibnegger G, et al. 43

Werner-Felmayer G, $\rightarrow$ Reibnegger $\mathrm{G}$, et al. 43

Werning C 580

Wernze $\mathrm{H}, \rightarrow$ Purucker E 1008

Wetzig $\mathrm{H}, \rightarrow$ Ludwig $\mathrm{M}$, et al. $\mathbf{5 7 0}$

Weyand CM, $\rightarrow$ Goronzy JJ 735

White $\mathrm{G}, \rightarrow$ Baumgartner FJ, et al. 619

Wichert $\mathrm{P}$ von, $\rightarrow$ Schmidt JA, et al. 191

Wicklmayr $\mathrm{M}$, $\rightarrow$ Stiegler $\mathrm{H}$, et al. 380

Wiedemann G, Müller-Klieser W, Walenta S, Schleinkofer L, Wood WG 33

Wiedemann G, $\rightarrow$ Jelkmann W 403

Wiegand V, Tracy S, Chapman $\mathrm{N}$, Wucherpfennig $\mathrm{C}$ 914

Wiegand $\mathrm{V}, \rightarrow$ Weidhase $\mathrm{A}$, et al. $\mathbf{8 8 0}$

Wieshammer S, Bode M, Weber L, Weismüller P, Binder $T$, Seibold H, Hombach V 436
Wilde V de, Vogelaers D, Colardyn $F \quad 615$

Williams $\mathrm{C}, \rightarrow$ Cotton PB 926

Wilms $\mathrm{B}, \rightarrow$ Eiff $\mathrm{M}$ von et al. 372

Wilske B, $\rightarrow$ Mayer W, et al. 431

Windeck $\mathrm{R} \quad 637$

Wirsching M 1177

Wolf $\mathrm{B}, \rightarrow$ Harrer $\mathrm{T}$, et al 864

Wolf $\mathrm{Ch}, \rightarrow$ Duntas L, et al. 1013

Wolff-Kormann PG, $\rightarrow$ Kormann BA, et al. 1027

Wood WG, Färber P, Kurowski V 324

Wood WG, $\rightarrow$ Wees J van, et al. 1054

Wood WG, $\rightarrow$ Wiedemann $\mathrm{G}$, et al. 33

Wucherpfennig $\mathrm{C}, \rightarrow$ Wiegand $\mathrm{V}$, et al. 914

Wüster C, Scholz A, Schmelzle A, Horowski R, Ziegler R 384

Yamanouchi $\mathrm{H}$, Shimada $\mathrm{H}$, Kuramoto K 1173

Yoshinari M, Kuroda T, Ikenoue $\mathrm{H}$, Okamura $\mathrm{K}$, Sato $\mathrm{K}$, Fujishima M 441

Zabel P, Horst H-J, Kreiker C, Schlaak M 1217

Zeitler E, Seyferth W 840

Ziegler R, $\rightarrow$ Feussner G, et al. 65,526

Ziegler R, $\rightarrow$ Franke-Raue K, et al. $\mathbf{5 9 7}$

Ziegler $\mathrm{R}, \rightarrow$ Wüster $\mathrm{C}$, et al. 384

Zimmerhackl LB, Fretschner M, Steinhausen M 166

Zimmermann $\mathrm{M}, \rightarrow$ Fu Q-G, et al. 125

Zoller WG, Hermans H, Bogner JR, Hahn D, Middeke M 830

Zoller WG, $\rightarrow$ Bogner JR, et al. $\mathbf{1 0 3 2}$

Zöllner N, Forth W 103, 105

Zöllner N, Gresser U, WalterSack I 101

Zöllner N, Sorg M, Krüger K 739

Zöllner N, $\rightarrow$ Cremer H-D, et al. 523

Zöllner $\mathrm{N}, \rightarrow$ Dörfler $\mathrm{H}$, et al. $\mathbf{3 8 8}$

Zöllner $\mathrm{N}, \rightarrow$ Gresser U, et al. 1222

Zöllner N, $\rightarrow$ Gross M, et al. 1084

Züchner $\mathrm{C}, \rightarrow$ Schrader $\mathrm{J}$, et al. 774

Redaktion: Springer-Verlag München, Agnes-Bernauer-Platz 8, W-8000 München 21

Schriftleitung: Prof. Dr. N. Zöllner, Medizinische Poliklinik der Universität München, Pettenkoferstr. 8 a, W-8000 München 2

Anzeigen: E. Lückermann, C. Hänzi, Springer-Verlag, Heidelberger Platz 3, 1000 Berlin 33

Druckerei: Universitätsdruckerei H. Stürtz AG, Beethovenstr. S, W-8700 Würzburg

(C) Springer-Verlag GmbH \& Co. KG, Berlin Heidelberg

Springer Verlag GmbH \& Co. KG, 1000 Berlin 33 


\title{
Erythrocyte Antibodies in AIDS Are Associated with Mycobacteriosis and Hypergammaglobulinemia
}

\author{
J.R. Bogner ${ }^{1}$, B. Gathof ${ }^{2}$, B. Heinrich ${ }^{1}$, A. Matuschke ${ }^{1}$, U. Bäcker ${ }^{2}$, and F.-D. Goebel ${ }^{1}$ \\ ${ }^{1}$ Medizinische Poliklinik, Universität München (Prof. Dr. N. Zöllner) \\ ${ }^{2}$ Blutspendedienst des Bayerischen Roten Kreuzes, München
}

Summary. Positive direct antiglobulin results prior to transfusion in some of our AIDS patients, as well as some reports in the literature on red cell antibodies in AIDS patients, prompted us to investigate the prevalence of erythrocyte antibodies in AIDS patients with transfusion requiring anemia. In addition we studied the question of relevant correlations with clinical diagnosis and with hematological and immunological laboratory parameters.

Of 145 consecutive hospitalized AIDS patients (CDC criteria), 34 (23\%) presented with anemia requiring transfusion. With each cross-match a routine antibody screening was performed. In cases of positive reaction additional antibody differentiation was done. Diagnoses, hematologic parameters, and therapy were studied retrospectively.

Agglutination was positive in at least one test for $41 \%(14 / 34)$ (group 1). C3d, anti I, cold agglutinins, and IgG occurred most frequently $(n=9 / 8 /$ $7 / 6$ out of 14). Seventyfive per cent (12/14) had leukopenia $(<4000 / \mu 1), 57 \%(8 / 14)$ had thrombocytopenia $(<150000 / \mu \mathrm{l})$, and $43 \%(6 / 14)$ showed both. Average values for leukocytes, thrombocytes, and CD4-positive lymphocytes did not differ significantly in patients with (group 1) and without (group 2) erythrocyte antibodies. Average gamma globulin levels were significantly increased in group $1(23.2 \mathrm{~g} / 1$ versus $16.9 \mathrm{~g} / 1 ; p<0.001)$.

In group $1,64 \%(9 / 14)$ had proven mycobacteriosis (6 atypical), in contrast to only $15 \%(3 / 20)$ in group $2(p<0.05)$. There were no significant differences between the two groups in prevalence of

Abbreviations: $\mathrm{ab}=$ antibodies $;$ AIDS $=$ acquired immunodeficiency syndrome; $\mathrm{ARC}=\mathrm{AIDS}$-related complex; $\mathrm{CD}=$ cluster of differentiation; $\mathrm{CDC}=$ centers for disease control; $\mathrm{CMV}=$ cytomegalovirus; DAT $=$ direct antiglobulin test HIV $=$ human immunodeficiency virus; $\mathrm{KS}=\mathrm{Kaposi}$ 's sarcoma; $\mathrm{NHL}=$ nonHodgkin's lymphoma; $\mathrm{PcP}=$ Pneumocystis carinii pneumonia other opportunistic infections, malignánt lymphoma, and Kaposi's sarcoma.

Autoimmune versus infectious pathogenesis of pathologic erythrocyte antibodies in AIDS has been discussed in the literature. Our results show an association of mycobacteriosis with positive direct antiglobulin test, suggesting that autoimmunological features might be triggered by certain pathogens.

Key words: Erythrocyte antibody - Direct antiglobulin test - Coombs' test - AIDS - Transfusion - Mycobacteriosis

Mild to profound anemia is seen in almost all AIDS patients with opportunistic infections. Anemia requiring transfusion is a common problem not only in patients receiving azidothymidine, but also in patients with severe infections. An abnormal positive direct antiglobulin test (DAT) has been observed in AIDS patients [6, 9, 11] as well as in asymptomatic HIV-infected patients [9]. It is not thought to contribute to significant clinical hemolysis and morbidity. Yet, life-threatening hemolytic complications can occur $[9,13]$.

Antibodies bound to the red cell membrane were detected in AIDS patients as early as 1981 . Toy and colleagues reviewed the transfusion service record at San Francisco General Hospital between 1981 and 1983 and found a prevalence of 18 percent [11]. DAT results were positive in $10 /$ 55 pretransfusion tests, which is a strikingly high prevalence compared with $0.6 \%$ positive DAT in the general hospital population [11]. Other reports confirmed this high prevalence, and figures range 
even higher, from 21 to 43 percent, in patients with AIDS $[6,8,15]$.

Some reports hint at an association of DAT with other hematological abnormalities such as leukopenia, lymphopenia, and immune thrombocytopenia $[4,6,11,15]$.

The first objective of our study was to compare the prevalence of eryhtrocyte - bound antibodies reported in the literature with our population of AIDS patients for such factors as geography.

Secondly, we investigated whether diagnosis and laboratory parameters (hematologic, immunologic) differ significantly in patients with and without erythrocyte antibodies.

\section{Patients and Methods}

Of 145 consecutive AIDS patients (diagnosis according to the criteria defined by the CDC) seen either at our outpatient clinic or admitted to our hospital, the 34 who presented between 1983 and 1988 with anemia requiring transfusion were retrospectively. All 34 patients were evaluated homoor bisexual and seropositive for anti HIV-1 in ELISA and Western Blot. Only one patient had received prior blood transfusions.

Cross-matches were done in $\mathrm{NaCl}-$, Bromelin-, and LISS-Coombs phase, with a control with the patient's own erythrocytes and serum in the LISSCoombs phase. With every cross-match a screening for erythrocyte antibodies was performed. If any of these tests results were positive, a further antibody differentiation was done with panels of 8 to 10 sorts of erythrocytes and/or with specific antisera for C3, C3d, C4, IgG, IgA, IgM, and IgD. All reagents used are commercially available in West Germany and licensed by the Food and Drug Administration of the United States.

The 34 patients tested were divided into two groups according to the result of the direct antiglobulin test: patients with positive erythrocyte antibodies, group 1, comprised patients with a positive agglutination test (reacting with at least one of the tested antibodies), whereas group 2 consisted of patients with all tests negative.

Clinical diagnoses of AIDS had been confirmed either microbiologically or by histological examination. Hematological parameters (leukocyte and thrombocyte counts), CD4-cell counts, and gamma globulin levels were taken from patients' records.

Statistical evaluation concerning the occurrence of opportunistic infections was performed by $\mathrm{X}^{2}$ test. Comparison of laboratory parameters was performed using a non-parametric statistical test (U-test, Mann-Whitney-Wilcoxon).
Table 1. Types of erythrocyte antibodies in 14 AIDS patients with anemia requiring transfusion. In addition to the most frequently occurring antibodies listed here, one patient was positive for anti $\mathrm{Le}^{\mathrm{a}}$ and another for anti $\mathrm{Jk}^{\mathrm{b}}$

\begin{tabular}{|c|c|c|c|c|c|c|c|}
\hline $\begin{array}{l}\text { Patient } \\
\text { No. }\end{array}$ & $\begin{array}{l}\text { direct } \\
\text { Coombs }\end{array}$ & $\mathrm{C} 3$ & $\mathrm{C} 3 \mathrm{~d}$ & $\mathrm{C} 4$ & Anti I & $\mathrm{IgG}$ & $\begin{array}{l}\text { Cold } \\
\text { Agglu- } \\
\text { tinins }\end{array}$ \\
\hline 1 & + & + & + & + & - & - & + \\
\hline 2 & + & - & + & - & - & + & - \\
\hline 3 & - & - & + & - & - & + & - \\
\hline 4 & + & - & + & - & + & + & + \\
\hline 5 & + & - & + & - & + & - & - \\
\hline 6 & - & - & - & - & - & - & + \\
\hline 7 & + & + & + & + & + & - & + \\
\hline 8 & - & - & - & - & + & - & - \\
\hline 9 & + & - & + & - & - & - & + \\
\hline 10 & + & - & + & - & - & - & + \\
\hline 11 & + & - & + & - & + & + & + \\
\hline 12 & + & + & + & - & - & + & - \\
\hline 13 & - & - & - & - & + & - & + \\
\hline 14 & + & - & + & - & - & + & - \\
\hline Total & 10 & 3 & 11 & 2 & 6 & 6 & 8 \\
\hline
\end{tabular}

\section{Results}

In $14 / 34(41 \%)$ of all patients tested, erythrocyte antibodies were detectable in at least one test (Table 1). Direct Coombs testing was positive in 10 of these 14 patients. C3 was found positive in $3 / 14$, $\mathrm{C} 3 \mathrm{~d}$ in 11 of $14, \mathrm{C} 4$ in 2 , anti $\mathrm{I}$ in $6, \mathrm{IgG}$ in 6 , and cold agglutinins in 8 of these 14 patients. A total of 12 patients showed a positive result in more than one test. Antibodies of the type anti $\mathrm{Le}^{\mathrm{a}}$ and anti $\mathrm{Jk}^{\mathrm{b}}$ were each found in one of 16 positive patients. One had had a transfusion 5 months earlier. Transfusion history was unknown in the one found positive for anti $\mathrm{Jk}^{\mathbf{b}}$.

Concomitant hematological abnormalities were present in both groups. In group 1 leukopenia was slightly more pronounced than in group 2 , with an average of $3200 / \mu \mathrm{l}$ versus $3972 / \mu \mathrm{l}$, but not statistically significantly. Thrombocytopenia was more marked in group 1 than in group $2(166000 / \mu l$ versus $192000 / \mu \mathrm{l})$. Signs of overt hemolysis were found in only two patients of group 1. Group 1 had an average absolute CD4-lymphocyte count of $62 / \mu \mathrm{l}$ as compared with $39 / \mu \mathrm{l}$ in group 2 (not significant). The level of gamma globulins was significantly higher in group $1(23.2 \mathrm{~g} / 1 \pm 13.3$ versus $16.9 \mathrm{~g} / 1 \pm 4.9 ; p<0.001)$. Data, averages, standard deviations, and levels of significance are given in Table 2 .

Clinical diagnosis. Nine of $14(64 \%)$ of the patients with either a positive DAT or erythrocyte anti- 
Table 2. Hematological, immunological, and clinical parameters of AIDS patients with anemia requiring transfusion (group 1) and without erythrocyte antibodies (group 2)

\begin{tabular}{|c|c|c|c|}
\hline \multirow[b]{2}{*}{ Leukocytes } & \multicolumn{3}{|c|}{ Group $1 n=14$ Group $2 n=20$} \\
\hline & $\begin{array}{c}3200 / \mu l \\
\pm 1208\end{array}$ & $\begin{array}{c}3972 / \mu l \\
\pm 1782\end{array}$ & n.s. \\
\hline Thrombocytes & $\begin{array}{c}166000 / \mu \mathrm{l} \\
\pm 126000\end{array}$ & $\begin{array}{c}192000 / \mu ! \\
\pm 140000\end{array}$ & n.s. \\
\hline CD4 + lymphocytes & $\begin{array}{l}61 / \mu l \\
\pm 34\end{array}$ & $\begin{array}{l}39 / \mu 1 \\
\pm 26\end{array}$ & n.s. \\
\hline Gamma globulins & $\begin{array}{l}23.2 \mathrm{~g} / 1 \\
\pm 13.3\end{array}$ & $\begin{array}{l}16.9 \mathrm{~g} / 1 \\
\pm 4.9\end{array}$ & $p<0.001$ \\
\hline Diagnosis $^{a}$ & $\begin{array}{l}\text { No. of } \\
\text { patients }\end{array}$ & $\begin{array}{l}\text { No. of } \\
\text { patients }\end{array}$ & \\
\hline Mycobacteriosis & 9 (6 atypical) & 3 (1 atypical) & $p<0.05$ \\
\hline $\mathrm{PcP}$ (and post-PcP) & 6 & 10 & n.s. \\
\hline CMV & 4 & 4 & n.s. \\
\hline $\begin{array}{l}\text { Toxoplasmosis, } \\
\text { cerebral }\end{array}$ & 1 & 1 & n.s. \\
\hline $\begin{array}{l}\text { Malignant } \\
\text { lymphoma }\end{array}$ & 2 & 4 & n.s. \\
\hline Kaposi's sarcoma & 3 & 3 & n.s. \\
\hline
\end{tabular}

a The number of patients totals more than 34 because of patients with two or more diagnoses; $\mathrm{PcP}=$ Pneumocystis carinii pneumonia, $\mathrm{CMV}=\mathrm{CMV}$ disease (retinitis, colitis, or encephalitis)

bodies had proven mycobacteriosis ( 3 had Mycobacterium tuberculosis; $3, \mathrm{M}$. avium intracellulare; 2 , M. fortuitum; and $1, \mathrm{M}$. xenopii) in contrast to only $15 \%$ of DAT-negative patients $(p<0.05)$.

Other diseases, such as Pneumocystis carinii pneumonia, cytomegalovirus disease, cerebral toxoplasmosis, non-Hodgkin's lymphoma, and Kaposi's sarcoma, occurred with the same frequency in the two groups (Table 2).

There was no obvious difference in treatment of the groups, especially with substances known to enhance the occurrence of a positive DAT.

\section{Discussion}

Reports on antibodies against blood cells of all types include studies not only on antibodies against thrombocytes [4, 7], granulocytes [4], and lymphocytes [2], but also on red cell autoantibodies [6, 12]. A high prevalence of a positive direct antiglobulin test and pathologic erythrocyte-bound antibodies in patients with AIDS and ARC, ranging between 18 and 43 percent, is reported in the literature $[8,11,14]$. This is in accordance with the $41 \%$ resulting in our study in the setting of transfusion in AIDS patients. This high prevalence resembles the prevalence of antierythrocyte antibodies in autoimmune disorders such as systemic lupus erythematosus, where prevalence can range from 10 to 40 percent, while hemolysis is less frequent [10]. Although there is a high prevalence of a positive DAT in AIDS patients, hemolysis rarely occurs $[9,11,13]$. In our patients only two of 14 with erythrocyte antibodies presented with overt hemolysis.

While only individuals with anemia requiring transfusion were tested, further investigation will be necessary to determine the correlation between autoantibodies and mycobacterial infections in patients with anemia not requiring transfusion or even without anemia.

In HIV-associated nephropathy there is clear evidence for geographic and racial variability. This fact prompted us to compare the prevalence found in our patients with the literature originating from North America. Our results show that there is no geographic difference in prevalence in patients with a diagnosis of AIDS according to the CDC criteria.

Lepennec and colleagues [5] found a red cell autoantibody prevalence of only $7.5 \%$ in asymptomatic HIV-infected patients. This, however, implies a variable prevalence when different stages of HIV infection are compared, e.g., lower prevalence in earlier stages. Further studies on the relation of prevalence to various clinical and pathogenetic stages of HIV infection are necessary to determine whether both erythrocyte-bound antibodies and antilymphocyte antibodies could contribute to morbidity in HIV infection.

C3d, anti I, IgG, and cold agglutinins were most frequently detected in our study. This is in accordance with the types of antibodies described in the literature, which include mainly anti I [6], anti $\operatorname{IgG}[6,11]$, and complement [12]. Again, this resembles the hemolytic anemia seen in autoimmune disorders.

Patients with erythrocyte-bound antibodies had significantly higher levels of gamma globulins in our study. This result is not at all surprising and confirms what was already known from studies by Toy [12] and Lepennec [5]. The pathogenetic meaning of the association of positive DAT with high immunoglobulin levels is not fully understood. It is suggested, however, that polyclonal immunoglobulin production might result in spontaneous immunoglobulin adherence to red cell membranes. In addition, positive DAT and the presence of circulating immune complexes were found by Inada and coworkers to correlate inversely (while 
sera and eluate contained no IgG antibodies against erythrocytes [3]). Perricone and colleagues give evidence for activation of complement in patients with ARC: $62 \%$ showed an impairment of classical and/or alternative complement pathway activity associated with the presence of cleavage products of $\mathrm{C} 3$ and a significant reduction of complement factors [7a]. Whether presence of erythrocyte-bound $\mathrm{C} 3 \mathrm{~d}$, e.g., is parallelled by low serum levels of $\mathrm{C} 3 \mathrm{~d}$ or other complement factors merits further investigation. Inada reported deposition of circulating immune complexes on erythrocytes via C3b receptors, which resulted in a defective clearing system for immune complexes on erythrocytes and in high membrane osmotic fragility [3]. This might be one of the mechanisms resulting in positive DAT in AIDS, as in autoimmune disorders and chronic infections resulting in hypergammaglobulinemia.

Erythrocyte antibodies may be induced (without respect to HIV) by certain pharmaceuticals such as phenacetin, methyldopa, and antibiotics. Because both groups of our patients were treated with such substances in similar frequency it is not probable that the pathogenesis of erythrocyte antibodies in AIDS is triggered by drugs. In all patients found to have a positive DAT, antimycobacterial therapy was started after the test.

Another pathophysiological explanation could be found in opportunistic infections with AIDS. Patients with ARC and AIDS are subject to latent, recurrent, and severe infections due to microorganisms known to cause red blood cell membrane modifications, including the exposure of novel carbohydrate cryptantigens that can react with naturally occurring antibodies [1]. Adams et al. tested 108 patients with ARC and AIDS for the most common forms of cryptantigens and found a high prevalence of binding site for peanut lectin [1]. An association of mycobacteriosis with DAT might be explained by such mechanisms. However, there are individuals with mycobacteriosis and negative DAT. Therefore, in our opinion, the high prevalence of erythrocyte-bound antibodies in AIDS is most probably due to a set of several possible triggering mechanisms, including autoimmune and infectious causes.

\section{References}

1. Adams M, Toy PT, Reid ME (1989) Exposure of cryptantigens on red blood cell membranes in patients with acquired immune deficiency syndrome or AIDS-related complex. J Acquir Immune Defic Syndr 2:224-228

2. Daniel V, Schimpf K, Opelz G (1988) Lymphocyte autoantibodies and alloantibodies in HIV-positive haemophilia patients. Clin Exp Immunol 75:178-183

3. Inada Y, Lange M, McKinley GF, Sonnabend JA, Fonville TW, Kanemitsu T, Tanaka M, Clark WS (1986) Hematologic correlates and the role of erythrocyte CR1 (C3b receptor) in the development of AIDS. AIDS Res Hum Retroviruses $2: 235-247$

4. van der Lelie J, Lange JM, Vos JJE, van Dalen CM, Danner SA, von dem Borne AE (1987) Autoimmunity against blood cells in human immunodeficiency virus (HIV) infection. $\mathrm{Br}$ J Haematol 67:109-114

5. Lepennec PY, Lefrere JJ, Rouzaud AM, Rouger P (1989) Red cell autoantibodies in asymptomatic HIV-infected subjects. Transfusion 29:465-466

6. McGinniss MH, Macher AM, Rook AH, Alter HJ (1986) Red cell autoantibodies in patients with acquired immune deficiency syndrome. Transfusion 26:405-409

7. Morris L, Distenfeld A, Amorosi E et al. (1982) Autoimmune thrombocytopenic purpura in homosexual men. Ann Intern Med 96:714-717

7a. Perricone R, Fontara L, deCarolis C, Carini C, Siriani MC, Aiuti F (1987) Evidence for activation of complement in patients with AIDS-related complex (ARC) and/or lymphadenopathy syndrome (LAS). Clin Exp Immunol 70:500-507

8. Raal FJ, Wypkema EM, Baynes RD (1988) Haematological abnormalities in the acquired immunodeficiency syndrome. S Afr Med J 74:157-160

9. Rapoport AP, Rowe JM, McMican A (1988) Life-threatening autoimmune hemolytic anemia in a patient with the acquired immune deficiency syndrome. Transfusion 28:190-191

10. Schoenfeld Y, Schwarz RS (1983) Hematologic manifestations. In: Schur PH (ed) The clinical management of systemic lupus erythematosus. Greene and Stratton, Orlando, Florida, pp 217-231

11. Toy PT, Reid ME, Burns M (1985) Positive direct antiglobulin test associated with hyperglobulinemia in acquired immunodeficiency syndrome (AIDS). Am J Hematol 19:145-150

12. Toy PT, Chin CA, Reid ME, Burns MA (1985) Factors associated with positive direct antiglobulin tests in pretransfusion patients: a case control study. Vox Sang 49:215-220

13. Zauber P, Park YK (1983) Neutropenia and hemolytic anemia in a homosexual man. Ann Intern Med 98:1029

14. Zon LI, Arkin C, Groopman JE (1987) Hematologic manifestations of the human immune deficiency virus (HIV). Br J Haematol 66:251-256

15. Zon LI, Groopman JE (1988) Hematologic manifestations of the human immune deficiency virus (HIV). Semin Hematol $25: 208-218$

Received: April 9, 1990

Returned for revision: June 8, 1990

Accepted: August 8, 1990

Dr. J.R. Bogner

Medizinische Poliklinik

Pettenkoferstraße 8 a

D-8000 München 2 